\title{
Stabilität durchströmter dünner magnetischer Flußröhren im Hinblick auf hohe Strömungsgeschwindigkeiten
}

\author{
Dissertation \\ zur Erlangung des Doktorgrades \\ der Mathematisch-Naturwissenschaftlichen Fakultäten \\ der Georg-August-Universität zu Göttingen
}

vorgelegt von

Bernd Jucknischke

aus Kassel

Göttingen 1999 
D7

Referent: Prof. Dr. W. Deinzer

Korreferent: Prof. Dr. D. Ronneberger

Tag der mündlichen Prüfung: 27. Januar 2000 


\section{Inhaltsverzeichnis}

$\begin{array}{lll}1 & \text { Motivation } & 4\end{array}$

2 Gleichgewicht 8

2.1 Modellierung und mathematische Beschreibung . . . . . . . . . . . . 8

2.2 Stehende Schocks im Gleichgewichtsmodell . . . . . . . . . . . . . . . . . . 14

2.3 Erhalt von Gleichgewichtslösungen $\ldots \ldots \ldots \ldots$

2.4 Ergebnisse . . . . . . . . . . . . . . . . . . . . . 17

$\begin{array}{llr}3 & \text { Stabilitätsanalyse } & 28\end{array}$

3.1 Störgrößen . . . . . . . . . . . . . . . . . . . . 28

3.2 Das beschreibende Gleichungssystem … . . . . . . . . . . . . . . . . . 29

3.2.1 Binormale Störungen . . . . . . . . . . . . . . . . . . . . . 39

3.2.2 Zeitabhängigkeit über Exponentialansatz . . . . . . . . . . . . . . . 39

3.3 Numerische Methoden . . . . . . . . . . . . . . . . . 40

3.3.1 Lösung des Eigenwertproblems mittels, ,Shooting ${ }^{\star}$. . . . . . . . . . . . . 43

3.3.2 Relaxation auf einem vorgegebenen Gitter . . . . . . . . . . . . . . 44

3.3 .3 Test der Numerik . . . . . . . . . . . . . . . . 47

3.4 Stabilitätskriterium aus den integrierten Stabilitätsgleichungen . . . . . . . . 47

3.4.1 Allgemeines Stabilitätskriterium . . . . . . . . . . . . . . . . 49

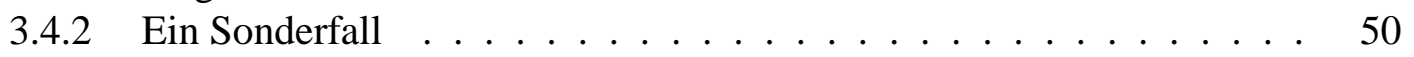

3.5 Ergebnisse . . . . . . . . . . . . . . . . . . . . . 52

3.5.1 Subkritische Strömungen . . . . . . . . . . . . . . 52

3.5 .2 Transkritische Strömung ohne Schock . . . . . . . . . . . . . . 53

3.5.3 Transkritische Strömung mit Schock . . . . . . . . . . . . . . 60

3.5.4 Superkritische Strömungen . . . . . . . . . . . . . . . . 68

$\begin{array}{lll}4 & \text { Zusammenfassung und Diskussion } & 78\end{array}$ 


\section{Motivation}

In der Astrophysik spielen magnetische Flußröhren eine bedeutende Rolle, die sich nicht auf Sterne oder im besonderen die Sonne beschränkt. Ein Beispiel dafür sind Jets von RadioGalaxien (Achterberg, 1988). Die Sonne nimmt aber aufgrund ihrer Nähe zu uns eine Sonderstellung ein; man kann auf ihr Phänomene beobachten, die sonst nicht oder nicht im Detail sichtbar wären.

So lassen sich z.B. Protuberanzen und ein 11-jähriger Sonnenfleckenzyklus beobachten.

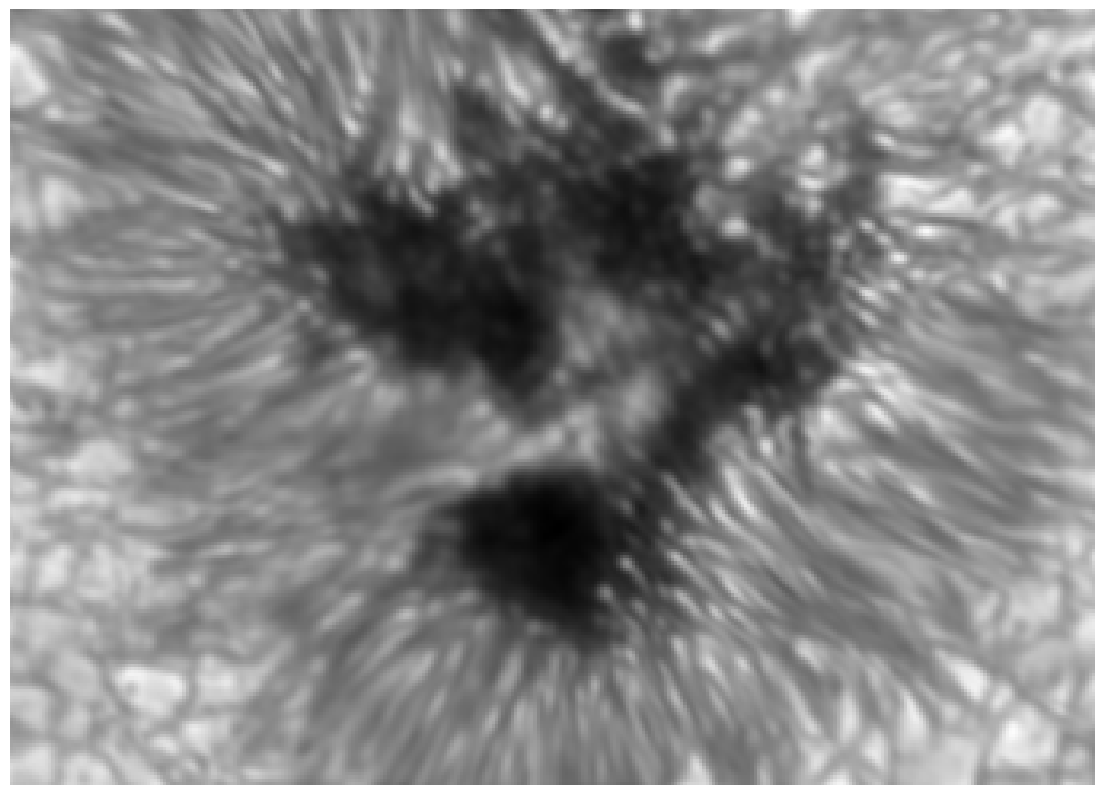

Abbildung 1.1: Sonnenfleck mit zentraler Umbra und filamentierter Penumbra

Über Magnetfeldmessungen findet man, daß die Periode des Sonnenfleckenzyklus eigentlich 22 Jahre beträgt und mit einen Magnetfeldzyklus einhergeht. Evershed (1909) hat bei der Beobachtung von Sonnenfleckenpenumbren eine systematische Linienverschiebung gefunden, die als eine parallel zur Sonnenoberfläche aus dem Sonnenfleck heraus verlaufende Plasmaströmung interpretiert wird.

Bei der Modellbildung zu den genannten Beobachtungen kommt jedesmal das Bild der magnetischen Flußröhre ins Spiel. Ruhende Protuberanzen stellt man sich als Ansammlung von ,kühler“ Materie in der „heißen“ Korona vor, die durch magnetische Kräfte am Hinabfallen auf die Sonnenoberfläche gehindert wird (Kippenhahn und Schlüter, 1957; Kuperus und Raadu, 1974; Degenhardt, 1995). Den Magnetfeldzyklus versucht man folgendermaßen 
zu verstehen: Ein Dynamo erzeugt großskaliges Magnetfeld in der Overshootregion unterhalb der Konvektionszone (Spiegel und Weiss, 1980; Prautzsch, 1997). Dieses steigt in Form von Flußröhren infolge einer Instabilität ab einer magnetischen Flußdichte von $\approx 10^{5}$ Gauss auf und ist verantwortlich für verschiedene auf der Sonnenoberfläche sichtbare Phänomene (Caligari et al., 1995). Für die Penumbra geht man von diesem Bild aus: Den hellen und dunklen Filamenten in einer Sonnenfleckenpenumbral werden unterschiedlich stark gegen die Sonnenoberfläche geneigte magnetische Flußröhren zugeordnet (Beckers und Schröter, 1969; Wiehr, 1999), wobei die auswärts gerichtete Strömung in den dunklen Filamenten verläuft. Die dunklen Filamente werden als bogenförmig gekrümmte, magnetische Flußröhren modelliert, die an anderer Stelle wieder auf die Sonnenoberfläche auftreffen. Zur Zeit nimmt man an, daß diese Auftreffpunkte sogenannte Filigree Elemente sind. Filigree Elemente sind helle Punkte auf der Sonnenoberfläche, die mit magnetischen Flußdichten von bis zu 3000 Gauss durchsetzt sind. Auch diese Filigree Elemente stellt man sich mit Hilfe der Modellvorstellung dünner magnetischer Flußröhren vor. Als Ursache der Strömung wird der Siphoneffekt angenommen (Meyer und Schmidt, 1968; Thomas und Montesinos, 1993) $)^{2}$.

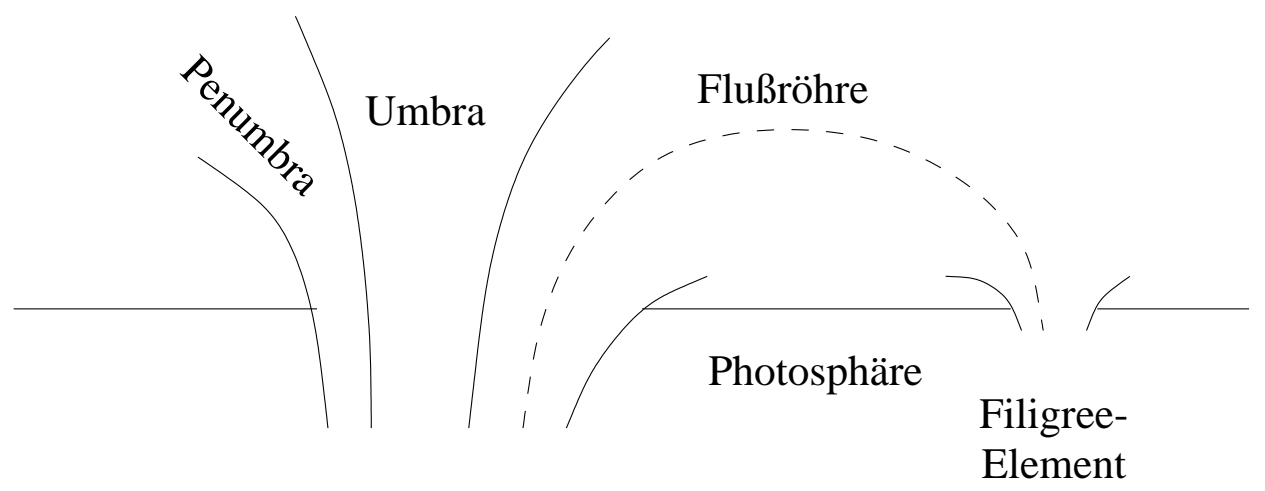

Sonnenfleck

Abbildung 1.2: Siphonströmung: In der Penumbra findet man auf gleicher Höhe über der Sonnenoberfläche ein vom Betrag her kleineres Magnetfeld als in einem Filigree Element. Aufgrund des durch Gleichung (2.10) gegebenen Druckgleichgewichts ist der Gasdruck in der Penumbra größer als in dem Filigree Element. Die Druckdifferenz treibt eine aus der Penumbra heraus gerichtete Strömung.

\section{Dünne Flußröhren}

Eine Flußröhre ist endlich ausgedehnt und umgeben von einem externen Medium. In beiden hängen die beobachtbaren Größen neben der Zeit im allgemeinen von den drei Ortskoordinaten ab. Damit ist eine Untersuchung im Rahmen der Magnetohydrodynamik (MHD) in der

\footnotetext{
${ }^{1}$ siehe Abb. 1.1

${ }^{2}$ siehe Abb. 1.2
} 
Regel nicht einfach. Oft genügt es jedoch, diese Flußröhren stark vereinfacht mit der Näherung dünner Flußröhren (NDF) in nullter Ordnung mathematisch als eine eindimensionale Raumkurve zu beschreiben. Die beobachtbaren Größen ändern sich in dieser Ordnung der Näherung über den Röhrenquerschnitt nicht. Die NDF wurde von Roberts und Webb (1978) für eine Flußröhre senkrecht zur Sonnenoberfläche entwickelt. Spruit (1981a; 1981b) hat die Näherung auf gekrümmte Flußröhren übertragen. Nimmt man darüber hinaus das externe Medium als räumlich nur von der Höhe über der Sonnenoberfläche abhängig an, so hat man eine deutliche Vereinfachung erreicht.

\section{Stabilitätsanalysen}

Ein großer Teil der erstellten Modelle, welche die NDF verwenden, ist statisch (Degenhardt, 1995) oder stationär (Degenhardt, 1989; Montesinos und Thomas, 1989; Degenhardt, 1991; Thomas und Montesinos, 1991), d.h. es werden entweder keine Bewegungen oder aber solche, die sich im Laufe der Zeit nicht ändern, zugelassen. Ob diese Modelle einer Störung widerstehen oder ihre Lebensdauer mit Beobachtungen verträglich ist, kann erst mit Stabilitätsuntersuchungen geklärt werden. Solche Untersuchungen waren im Rahmen der MHD in der Vergangenheit recht aufwendig (Schüßler, 1990; Degenhardt, 1995).

Schmitt (1995; 1998) hat einen Formalismus entwickelt, der für lineare Stabilitätsanalysen die beschreibenden Gleichungen in einer speziellen Form angibt. Schreibt man die Gleichungen in Operatorschreibweise, so läßt sich, wenn geeignete Randbedingungen verwendet werden, die Hermitezität der Operatoren zeigen. Wegen dieser Operatoreigenschaft wird die Entscheidung, ob ein untersuchtes Gleichgewichtsmodell stabil ist, vereinfacht: Sind alle bei der Lösung der beschreibenden Gleichungen erhaltenen Eigenwerte reell, ist das untersuchte Modell stabil, sonst ist es instabil. In den genannten Veröffentlichungen hat Schmitt auch Anwendungen seines Formalismus gezeigt; unter anderem hat er sich mit der Stabilität des von Degenhardt (1990) vorgestellten Modells einer Siphonströmung zur Deutung des EvershedEffekts befaßt. In dieser Untersuchung hat Schmitt sich auf unterkritische Strömungen beschränkt, bei denen die Strömungsgeschwindigkeit überall kleiner ist als die durch Gleichung (2.17) definierte kritische Geschwindigkeit $c_{T}$.

\section{Ziel und Gliederung dieser Arbeit}

In der vorliegenden Arbeit soll untersucht werden, ob sich der Schmittsche Formalismus auch auf Gleichgewichtsmodelle mit hohen Strömungsgeschwindigkeiten anwenden läßt. Diese lassen sich in unterschiedliche Klassen einteilen

- Die schon erwähnten sub- oder unterkritischen Strömungen

- transkritische Strömungen: Die Strömungsgeschwindigkeit ist teilweise unter-, teilweise überkritisch und erreicht an wenigstens einem Punkt die kritische Geschwindigkeit. In dieser Arbeit werden zwei Arten von transkritischen Strömungen betrachtet 
- transkritische Strömungen ohne Schock: Die Strömungsgeschwindigkeit steigt im Verlauf der Strömung, beginnend bei unterkritischen Werten, über die kritische Geschwindigkeit an und erreicht schließlich überkritische Werte.

- transkritische Strömungen mit Schock: wie oben, nur wird in Strömungsrichtung kurz nach dem Erreichen der kritischen Geschwindigkeit ein Schock eingeführt, der dafür sorgt, daß am Punkt des Wiederauftreffens der Röhre auf die Sonnenoberfläche die beobachtbaren Größen realistische Werte annehmen.

- super- oder überkritische Strömungen: Die Strömungsgeschwindigkeit ist an jedem Punkt größer als die kritische Geschwindigkeit.

Um sich auf auf die Auswirkungen der Strömungsgeschwindigkeit innerhalb der Flußröhre konzentrieren zu können wird für die Stabilitätsanalyse nicht das mit Blick auf Nähe zur Realität konstruierte Gleichgewichtsmodell von Degenhardt (1990) verwendet, sondern in Abschnitt 2 ein einfacheres, sich an der Arbeit von Thomas und Montesinos (1991) orientierendes Gleichgewichtsmodell einer Siphonströmung abgeleitet. Dieses Modell unterscheidet sich in drei Punkten vom Degenhardtschen:

- Es wird eine einfachere, zur Beschreibung hoher Strömungsgeschwindigkeiten geeignete Energiegleichung verwendet.

- Die Flußröhre wird in eine isotherme Umgebung eingebettet.

- Im Fall einer transkritischen Strömung kann ein als Diskontinuität behandelter Schock mit einbezogen werden.

Ausgewählte Ergebnisse dieses Modells werden präsentiert und in Kapitel 3 auf ihre Stabilität hin untersucht. In diesem Abschnitt wird auch auf die im Zusammenhang mit an kritischen Punkten auftretenden Singularitäten der Stabilitätsgleichungen zu bewältigenden Probleme eingegangen. Die verwendeten numerischen Methoden werden dargelegt und Ergebnisse der Stabilitätsanalyse vorgestellt. In Kapitel 4 erfolgt die Diskussion, in der eine Zusammenfassung der Ergebnisse dieser Arbeit und ein Ausblick auf wünschenswerte Erweiterungen gegeben wird. 


\section{Gleichgewicht}

In diesem Abschnitt soll, basierend auf den Gleichungen der MHD für den Fall idealer Leitfähigkeit, eine stationär durchströmte dünne Flußröhre modelliert werden. Das Modell wird alsdann zur Interpretation des Evershed-Effekts herangezogen.

\subsection{Modellierung und mathematische Beschreibung}

\section{Grundgleichungen}

Als Ausgangspunkt für das Modell dienen die Gleichungen der MHD, wie man sie z.B. in Kippenhahn und Möllenhoff (1975) findet. Diese Gleichungen sind hier im Gaußschen cgsSystem angegeben und bereits auf eine Form gebracht, die ideale Leifähigkeit und Stationarität der Strömung berücksichtigt.

$$
\begin{aligned}
\nabla \times(\vec{v} \times \vec{B}) & =0 \\
\nabla \cdot \vec{B} & =0 \\
(\vec{v} \cdot \nabla) \vec{v} & =-\frac{\nabla P}{\rho}+\frac{(\nabla \times \vec{B}) \times \vec{B}}{4 \pi \rho}+\vec{g} \\
\nabla \cdot(\rho \vec{v}) & =0 \\
P & =P(\rho, T)
\end{aligned}
$$

$\vec{v}$ und $\vec{B}$ sind die Vektoren der Strömungsgeschwindigkeit und magnetischen Flußdichte, $P$ ist der Gasdruck, $\rho$ die Dichte und $\vec{g}$ der Vektor der Schwerebeschleunigung. $T$ schließlich ist die Temperatur. Alle genannten Größen gelten im Inneren der zu modellierenden Flußröhre. Das Gleichungssystem wird durch eine Energiegleichung geschlossen.

In dieser Arbeit liegt der Schwerpunkt auf der Stabilitätsanalyse von mit hoher Geschwindigkeit durchströmten Flußröhren. Um sich auf den Effekt der Strömungsgeschwindigkeit konzentrieren zu können, ist es sinnvoll, ein einfaches Gleichgewichtsmodell zu erstellen. Ein solches Modell kann natürlich nur eine grobe Beschreibung der „Wirklichkeit“ geben. Im Hinblick auf hohe Strömungsgeschwindigkeiten wird als Energiegleichung die Polytropengleichung gewählt.

$$
\frac{P}{P_{0}}=\left(\frac{\rho}{\rho_{0}}\right)^{\alpha}
$$


$\alpha$ ist der Quotient der spezifischen Wärmen, und mit Index „0“ versehene Größen bezeichnen Anfangswerte, die am linken Fußpunkt der betrachteten Flußröhre vorgegeben werden. Aufgrund dieser Wahl der Energiegleichung dient die Zustandsgleichung (2.2) nur noch der Bestimmung der Temperatur. Sie wird im weiteren nicht mehr mit aufgeführt.

\section{Die Näherung dünner Flußröhren}

Bei einer dünnen Flußröhre ist der Durchmesser, verglichen mit allen physikalisch wichtigen Skalen, vernachlässigbar klein. In der hier verwendeten nullten Ordnung der Näherung hängen die physikalischen Größen in der Röhre räumlich nur von der Koordinate entlang der Röhre ab. Eine mathematisch sinnvolle Beschreibung ist dann die einer Raumkurve. Die Einheitsvektoren $\hat{t}, \hat{n}$ und $\hat{b}$ bilden das begleitende Dreibein der Röhre; sie sind definiert als

$$
\hat{t}=\left(\begin{array}{c}
d x / d s \\
d y / d s \\
d z / d s
\end{array}\right) ; \quad \hat{n}=R_{C}\left(\begin{array}{c}
d^{2} x / d s^{2} \\
d^{2} y / d s^{2} \\
d^{2} z / d s^{2}
\end{array}\right) ; \quad \hat{b}=\hat{t} \times \hat{n}
$$

und weisen in die Richtungen tangential, normal und binormal zur Flußröhre; $x, y$, und $z$ sind die Cartesischen Komponenten eines Ortsvektors an die Röhre (Bourne und Kendall, 1973, S. 69). Das Differential der Bogenlänge $d s$ und der Krümmungsradius $R_{C}$ sind gegeben durch

$$
\begin{aligned}
d s^{2} & =d x^{2}+d y^{2}+d z^{2} \\
\frac{1}{R_{C}^{2}} & =\left(\frac{d^{2} x}{d s^{2}}\right)^{2}+\left(\frac{d^{2} y}{d s^{2}}\right)^{2}+\left(\frac{d^{2} z}{d s^{2}}\right)^{2}
\end{aligned}
$$

Die Krümmung der Sonnenoberfläche werde vernachlässigt, und der Vektor $\vec{g}$ der Gravitationsbeschleunigung sei konstant, vom Betrag $g$ und antiparallel zum vertikal auf der Sonnenoberfläche stehenden Einheitsvektor $\hat{z}: \vec{g}=-g \hat{z}$. Diese Näherungen sind infolge der beobachteten räumlichen Ausdehnung der penumbralen Filamente gerechtfertigt. Es folgen die skalaren Produkte

$$
\begin{aligned}
\vec{g} \cdot \hat{t} & =-g \frac{d z}{d s} \\
\vec{g} \cdot \hat{n} & =-g R_{C} \frac{d^{2} z}{d s^{2}}
\end{aligned}
$$

Für Strömungsgeschwindigkeit und magnetische Flußdichte gelten

$$
\begin{aligned}
\vec{v} & =v \hat{t} \\
\vec{B} & =B \hat{t}
\end{aligned}
$$


Setzt man die Vektoridentität (Bourne und Kendall, 1973, S. 101)

$$
(\nabla \times B \hat{t}) \times B \hat{t}=(B \hat{t} \cdot \nabla)(B \hat{t})-\frac{1}{2} \nabla B^{2}
$$

in die Bewegungsgleichung (2.1) ein, so wird diese $\mathrm{zu}$

$$
(v \hat{t} \cdot \nabla)(v \hat{t})=-\frac{\nabla\left(P+B^{2} / 8 \pi\right)}{\rho}+\frac{(B \hat{t} \cdot \nabla)(B \hat{t})}{4 \pi \rho}+\vec{g}
$$

Wegen

$$
\hat{t} \cdot \nabla=\frac{d x}{d s} \frac{\partial}{\partial x}+\frac{d y}{d s} \frac{\partial}{\partial y}+\frac{d z}{d s} \frac{\partial}{\partial z}=\frac{d}{d s}
$$

wird

$$
(v \hat{t} \cdot \nabla)(v \hat{t})=v \frac{d(v \hat{t})}{d s}=v \frac{d v}{d s} \hat{t}+v^{2} \frac{d \hat{t}}{d s}=\frac{1}{2} \frac{d v^{2}}{d s} \hat{t}+\frac{v^{2}}{R_{C}} \hat{n}
$$

Analog gilt

$$
(B \hat{t} \cdot \nabla)(B \hat{t})=\frac{1}{2} \frac{d B^{2}}{d s} \hat{t}+\frac{B^{2}}{R_{C}} \hat{n}
$$

Das läßt sich in die Bewegungsgleichung (2.6) einsetzen, und man erhält für das Gleichungssystem

$$
\begin{aligned}
\nabla \cdot(B \hat{t}) & =0 \\
{\left[\frac{1}{2} \frac{d v^{2}}{d s}-\frac{1}{\rho} \frac{d}{d s}\left(\frac{B^{2}}{8 \pi}\right)\right] \hat{t}+\left(v^{2}-\frac{B^{2}}{4 \pi \rho}\right) \frac{\hat{n}}{R_{C}} } & =-\frac{1}{\rho} \nabla\left(P+\frac{B^{2}}{8 \pi}\right)+\vec{g} \\
\nabla \cdot(\rho v \hat{t}) & =0 \\
P & =P_{0}\left(\frac{\rho}{\rho_{0}}\right)^{\alpha}
\end{aligned}
$$

\section{Eine Erhaltungsgröße}

Aus der Divergenzfreiheit des Magnetfelds (2.7) folgt

$$
\nabla \cdot \hat{t}=-\hat{t} \cdot \frac{\nabla B}{B}
$$

Damit kann die Kontinuitätsgleichung (2.8) in folgende Form gebracht werden

$$
\frac{d(\rho v)}{d s}-\frac{\rho v}{B} \frac{d B}{d s}=0
$$


Die Größe $\rho v / B$ hängt also nicht von der Bogenlänge $s$ ab. Es wird

$$
v^{2}=\frac{B^{2}}{\chi_{0} \rho^{2}}=\frac{4 \pi}{\chi_{0} \rho} \frac{B^{2}}{4 \pi \rho}=\frac{4 \pi}{\chi_{0} \rho} v_{A}^{2}
$$

mit $\chi_{0}=B_{0}^{2} /\left(\rho_{0}^{2} v_{0}^{2}\right)$ und $v_{A}^{2}=B^{2} /(4 \pi \rho) ; v_{A}$ heißt Alfvéngeschwindigkeit. Man erhält

$$
\frac{d v^{2}}{d s}=\frac{1}{\chi_{0}}\left(\frac{1}{\rho^{2}} \frac{d B^{2}}{d s}-\frac{2 B^{2}}{\rho^{3}} \frac{d \rho}{d s}\right)=2 \frac{4 \pi}{\chi_{0} \rho} \frac{1}{\rho}\left[\frac{d}{d s}\left(\frac{B^{2}}{8 \pi}\right)-v_{A}^{2} \frac{d \rho}{d s}\right]
$$

Das Gleichungssystem hat nun die Form

$$
\begin{aligned}
\frac{1}{\rho}\left[\left(\frac{4 \pi}{\chi_{0} \rho}-1\right) \frac{d}{d s}\left(\frac{B^{2}}{8 \pi}\right)-\frac{4 \pi}{\chi_{0} \rho} v_{A}^{2} \frac{d \rho}{d s}\right] \hat{t}+\left(\frac{4 \pi}{\chi_{0} \rho}-1\right) \frac{v_{A}^{2}}{R_{C}} \hat{n} & =-\frac{1}{\rho} \nabla\left(P+\frac{B^{2}}{8 \pi}\right)+\vec{g} \\
P & =P_{0}\left(\frac{\rho}{\rho_{0}}\right)^{\alpha}
\end{aligned}
$$

\section{Druckgleichgewicht zwischen Flußröhre und umgebendem Medium}

Auf der Grenze zwischen Flußröhre und umgebendem Medium gilt die Gleichheit des Druckes

$$
P+\frac{B^{2}}{8 \pi}=P_{e}+\frac{B_{e}^{2}}{8 \pi}
$$

Wegen der hier verwendeten nullten Ordnung der NDF gilt diese Gleichung für den gesamten Querschnitt der Röhre. Größen mit Index ,,e “ bezeichnen die Werte der physikalischen Veränderlichen im externen Medium. Die magnetische Flußdichte außerhalb der Flußröhre wird als konstant angenommen. Durch ihre Einführung soll auf einfache Art dem penumbralen Magnetfeld Rechnung getragen werden (Thomas und Montesinos, 1993). Differenziert man Gleichung (2.10) nach $s$, so wird unter Verwendung der Polytropengleichung (2.3)

$$
\frac{d}{d s}\left(\frac{B^{2}}{8 \pi}\right)=\frac{d P_{e}}{d s}-\frac{\alpha P_{0}}{\rho_{0}}\left(\frac{\rho}{\rho_{0}}\right)^{\alpha-1} \frac{d \rho}{d s}
$$

Das Quadrat der Schallgeschwindigkeit ist $c_{S}^{2}=\alpha P / \rho$, man erhält

$$
c_{S}^{2}=\frac{\alpha}{\rho} P_{0}\left(\frac{\rho}{\rho_{0}}\right)^{\alpha}=\frac{\alpha P_{0}}{\rho_{0}}\left(\frac{\rho}{\rho_{0}}\right)^{\alpha-1}
$$

und es ergibt sich

$$
\frac{d}{d s}\left(\frac{B^{2}}{8 \pi}\right)=\frac{d P_{e}}{d s}-c_{S}^{2} \frac{d \rho}{d s}
$$


Das in der Bewegungsgleichung auftretende Quadrat der Alfvéngeschwindigkeit kann nun geschrieben werden als

$$
v_{A}^{2}=\frac{2}{\rho} \frac{B^{2}}{8 \pi}=\frac{2}{\rho}\left[P_{e}+\frac{B_{e}^{2}}{8 \pi}-P_{0}\left(\frac{\rho}{\rho_{0}}\right)^{\alpha}\right]
$$

Das Gleichungssystem ist auf die Bewegungsgleichung reduziert; diese lautet in Komponenten entlang, normal und binormal zur Flußröhre

$$
\begin{aligned}
\frac{1}{\rho}\left[\left(\frac{4 \pi}{\chi_{0} \rho}-1\right) \frac{d P_{e}}{d s}-\left(\frac{4 \pi}{\chi_{0} \rho}-1\right) c_{S}^{2} \frac{d \rho}{d s}-\frac{4 \pi}{\chi_{0} \rho} v_{A}^{2} \frac{d \rho}{d s}\right] & =-\left[\frac{1}{\rho} \nabla\left(P_{e}+\frac{B_{e}^{2}}{8 \pi}\right)-\vec{g}\right] \cdot \hat{t} \\
\left(\frac{4 \pi}{\chi_{0} \rho}-1\right) \frac{v_{A}^{2}}{R_{C}} & =-\left[\frac{1}{\rho} \nabla\left(P_{e}+\frac{B_{e}^{2}}{8 \pi}\right)-\vec{g}\right] \cdot \hat{n} \\
0 & =-\left[\frac{1}{\rho} \nabla\left(P_{e}+\frac{B_{e}^{2}}{8 \pi}\right)-\vec{g}\right] \cdot \hat{b}
\end{aligned}
$$

\section{Die Schichtung des umgebenden Mediums}

Das externe Medium sei isotherm, gravitativ geschichtet und werde als magnetohydrostatisch angenommen. Dann gilt wegen der als räumlich unveränderlich angenommenen magnetischen Flußdichte dort

$$
\nabla\left(P_{e}+\frac{B_{e}^{2}}{8 \pi}\right)=\rho_{e} \vec{g}
$$

Mit dieser Gleichung erkennt man, daß die rechten Seiten im Gleichungssystem (2.11) skalare Produkte von Auftriebskraft mit den Einheitsvektoren entlang, normal und binormal zur Flußröhre sind. Weiter ist

$$
\frac{d P_{e}}{d s}=\frac{d P_{e}}{d z} \frac{d z}{d s}=-g \rho_{e} \frac{d z}{d s}
$$

Wegen der Isothermie gilt

$$
\rho_{e}=\frac{P_{e}}{P_{e 0}} \rho_{e 0}
$$

Die Komponenten der Bewegungsgleichung werden unter Verwendung der Gleichungen (2.5) $\mathrm{zu}$

$$
\begin{aligned}
-\frac{1}{\rho}\left[\left(\frac{4 \pi}{\chi_{0} \rho}-1\right) c_{S}^{2}+\frac{4 \pi}{\chi_{0} \rho} v_{A}^{2}\right] \frac{d \rho}{d s} & =g\left(\frac{4 \pi}{\chi_{0} \rho} \frac{\rho_{e}}{\rho}-1\right) \frac{d z}{d s} \\
\frac{v_{A}^{2}}{R_{C}}\left(\frac{4 \pi}{\chi_{0} \rho}-1\right) & =g\left(\frac{\rho_{e}}{\rho}-1\right) R_{C} \frac{d^{2} z}{d s^{2}} \\
0 & =\left(\frac{\rho_{e}}{\rho}-1\right) \vec{g} \cdot \hat{b}
\end{aligned}
$$




\section{Strömung in einer Ebene senkrecht zur Sonnenoberfläche}

Die letzte Gleichung ist erfüllt, wenn der Auftrieb verschwindet oder die Flußröhre in einer Ebene liegt, die den Vektor $\vec{g}$ enthält. Hier wird letzterer Fall verfolgt. Dann gelten für den Krümmungsradius $R_{C}$ und das Differential der Bogenlänge $d s$ vereinfachte Formen der Gleichungen im System (2.4), nämlich

$$
\begin{aligned}
\frac{1}{R_{C}^{2}} & =\left(\frac{d^{2} x}{d s^{2}}\right)^{2}+\left(\frac{d^{2} z}{d s^{2}}\right)^{2} \\
d s^{2} & =d x^{2}+d z^{2}
\end{aligned}
$$

Man kann $x$ eliminieren! und erhält

$$
\frac{1}{R_{C}^{2}}=\frac{\left(d^{2} z / d s^{2}\right)^{2}}{1-(d z / d s)^{2}}
$$

\section{Das zu lösende Gleichungssystem}

Das Resultat der obigen Umformungen sind die $\hat{t}$ - und $\hat{n}$-Komponenten der Bewegungsgleichung, aufgelöst nach $d \rho / d s$ und $d^{2} z / d s^{2}$

$$
\begin{aligned}
\frac{d \rho}{d s} & =\frac{g\left(\frac{\chi_{0} \rho}{4 \pi}-\frac{\rho_{e}}{\rho}\right) \frac{d z}{d s}}{\frac{1}{\rho}\left[\left(1-\frac{\chi_{0} \rho}{4 \pi}\right) c_{S}^{2}+v_{A}^{2}\right]} \\
\frac{d^{2} z}{d s^{2}} & =\frac{g\left(\frac{\rho_{e}}{\rho}-1\right)\left[1-\left(\frac{d z}{d s}\right)^{2}\right]}{\left(\frac{4 \pi}{\chi_{0} \rho}-1\right) v_{A}^{2}} \vee \frac{d^{2} z}{d s^{2}}=0
\end{aligned}
$$

und eine Gleichung, die den Gasdruck im äußeren Medium in Abhängigkeit von der Bogenlänge ermittelt

$$
\frac{d P_{e}}{d s}=-g \rho_{e} \frac{d z}{d s}
$$

Hilfsvariable sind die drei Größen

$$
\begin{aligned}
c_{S}^{2} & =\frac{\alpha P_{0}}{\rho_{0}}\left(\frac{\rho}{\rho_{0}}\right)^{\alpha-1} \\
v_{A}^{2} & =\frac{2}{\rho}\left[P_{e}+\frac{B_{e}^{2}}{8 \pi}-P_{0}\left(\frac{\rho}{\rho_{0}}\right)^{\alpha}\right] \\
\rho_{e} & =\frac{P_{e}}{P_{e 0}} \rho_{e 0}
\end{aligned}
$$

${ }^{1}$ Bei der Rechnung wird $d x / d s \neq 0$ vorausgesetzt. 


\section{Kritische Geschwindigkeiten}

Der Nenner von Gleichung (2.13) verschwindet, falls

$$
\left(1-\frac{\chi_{0} \rho}{4 \pi}\right) c_{S}^{2}+v_{A}^{2}=0
$$

Unter Verwendung von Gleichung (2.9) läßt sich zeigen, daß dann gilt

$$
v^{2}=\frac{c_{S}^{2} v_{A}^{2}}{c_{S}^{2}+v_{A}^{2}}=c_{T}^{2}
$$

Die zugehörige Strömungsgeschwindigkeit $v$ heißt „kritische Geschwindigkeit“ und wird in der englischsprachigen Literatur als „tube speed“ bezeichnet.

Auch in Gleichung (2.14) kann der Nenner den Wert Null annehmen. Dies tritt ein für

$$
\frac{4 \pi}{\chi_{0} \rho}=1
$$

Gleichung (2.9) kann entnommen werden, daß dann für die Strömungsgeschwindigkeit gilt $v=v_{A}$. Bei den in dieser Arbeit untersuchten Penumbraströmungen ist die Strömungsgeschwindigkeit stets deutlich unteralfvénisch, darum wird dieser Fall nicht weiter untersucht.

\subsection{Stehende Schocks im Gleichgewichtsmodell}

Thomas und Montesinos (1991) haben transkritische, stationäre Strömungen mit stehenden Schocks untersucht. Die starke Änderung der physikalischen Größen auf einer im Vergleich zum Flußröhrendurchmesser kleinen räumlichen Skala, die mit einem Schock einhergeht, sprengt die NDF. Die detaillierte Struktur eines Schocks aufzulösen, ist damit unter den verwendeten Modellannahmen nicht möglich. In der Literatur findet sich die Möglichkeit, den Schock als eine Diskontinuität zu betrachten (Herbold et al., 1985; Ferriz-Mas und MorenoInsertis, 1987). In dieser Arbeit werden folgende Übergangsbedingungen, die eine polytrope Änderung der physikalischen Größen über die Diskontinuität beschreiben, verwendet

$$
\begin{aligned}
\rho_{l} v_{l} A_{l} & =\rho_{r} v_{r} A_{r} \\
A_{l}\left(\frac{\rho_{l} v_{l}^{2}}{2}+P_{l}-P_{e}-\frac{B_{e}^{2}}{8 \pi}\right) & =A_{r}\left(\frac{\rho_{r} v_{r}^{2}}{2}+P_{r}-P_{e}-\frac{B_{e}^{2}}{8 \pi}\right) \\
\frac{v_{l}^{2}}{2}+\frac{\alpha}{\alpha-1} \frac{P_{l}}{\rho_{l}} & =\frac{v_{r}^{2}}{2}+\frac{\alpha}{\alpha-1} \frac{P_{r}}{\rho_{r}} \\
B_{l} A_{l} & =B_{r} A_{r} \\
P_{l}+\frac{B_{l}^{2}}{8 \pi} & =P_{r}+\frac{B_{r}^{2}}{8 \pi}=P_{e}+\frac{B_{e}^{2}}{8 \pi} \\
S_{l} & \leq S_{r}
\end{aligned}
$$


$A$ ist der Querschnitt der Flußröhre und $S$ die Entropie. Die Indizes „l“ und „,r“ stehen für links bzw. rechts vom Schock? Diese Bedingungen sind bis auf einen Term in der zweiten Gleichung, der einem äußeren Magnetfeld Rechnung tragen soll, identisch mit denen, die Thomas \& Montesinos (1991) verwendet haben.

\section{Vereinfachen der Übergangsbedingungen ${ }^{\text {而 }}$}

Wegen der vierten Gleichung im System (2.18) ist das Verhältnis der Querschnittsflächen links und rechts vom Schock bekannt. Damit wird

$$
\begin{aligned}
\rho_{l} v_{l} \frac{B_{r}}{B_{l}} & =\rho_{r} v_{r} \\
\frac{B_{r}}{B_{l}}\left(\frac{\rho_{l} v_{l}^{2}}{2}+P_{l}-P_{e}-\frac{B_{e}^{2}}{8 \pi}\right) & =\frac{\rho_{r} v_{r}^{2}}{2}+P_{r}-P_{e}-\frac{B_{e}^{2}}{8 \pi}
\end{aligned}
$$

Aufgrund der ersten dieser beiden Gleichungen läßt sich $\rho_{r}$ ersetzten. Es ergibt sich

$$
\begin{aligned}
\frac{B_{r}}{B_{l}}\left(\frac{\rho_{l} v_{l}^{2}}{2}+P_{l}-P_{e}-\frac{B_{e}^{2}}{8 \pi}\right) & =\frac{B_{r}}{B_{l}} \frac{\rho_{l} v_{l} v_{r}}{2}+P_{r}-P_{e}-\frac{B_{e}^{2}}{8 \pi} \\
\frac{v_{l}^{2}}{2}+\frac{\alpha}{\alpha-1} \frac{P_{l}}{\rho_{l}} & =\frac{v_{r}^{2}}{2}+\frac{\alpha}{\alpha-1} \frac{P_{r} v_{r} B_{l}}{\rho_{l} v_{l} B_{r}}
\end{aligned}
$$

Mit der fünften Gleichung des Systems (2.18) kann man $P_{r}$ eliminieren. Man erhält

$$
\begin{aligned}
\frac{\rho_{l} v_{l}^{2}}{2}-\frac{B_{l}^{2}}{8 \pi} & =\frac{\rho_{l} v_{l} v_{r}}{2}-\frac{B_{l} B_{r}}{8 \pi} \\
\frac{v_{l}^{2}}{2}+\frac{\alpha}{\alpha-1} \frac{P_{l}}{\rho_{l}} & =\frac{v_{r}^{2}}{2}+\frac{\alpha}{\alpha-1}\left(P_{l}+\frac{B_{l}^{2}}{8 \pi}-\frac{B_{r}^{2}}{8 \pi}\right) \frac{v_{r} B_{l}}{\rho_{l} v_{l} B_{r}}
\end{aligned}
$$

Mit Hilfe der ersten dieser beiden Gleichungen läßt sich $v_{r}$ angeben, und es folgt

$$
\begin{aligned}
0= & B_{r}^{3} \frac{1}{4 \pi \rho_{l} v_{l}^{2}} \frac{B_{l}}{8 \pi}\left(1-\frac{\alpha}{\alpha-1}\right)+ \\
& +B_{r}^{2} \frac{1}{4 \pi}\left(1-\frac{B_{l}^{2}}{4 \pi \rho_{l} v_{l}^{2}}\right)\left(1-\frac{1}{2} \frac{\alpha}{\alpha-1}\right)+ \\
& +B_{r} \frac{1}{B_{l}}\left\{-\frac{\alpha}{\alpha-1} P_{l}\left(1-\frac{B_{l}^{2}}{4 \pi \rho_{l} v_{l}^{2}}\right)-\frac{B_{l}^{2}}{4 \pi}\left[1-\frac{B_{l}^{2}}{8 \pi \rho_{l} v_{l}^{2}}\left(1+\frac{\alpha}{\alpha-1}\right)\right]\right\}+ \\
& +\frac{\alpha}{\alpha-1}\left(P_{l}+\frac{B_{l}^{2}}{8 \pi}\right)\left(1-\frac{B_{l}^{2}}{4 \pi \rho_{l} v_{l}^{2}}\right)
\end{aligned}
$$

\footnotetext{
${ }^{2}$ Die Strömung läuft von links nach rechts, ist also unmittelbar links vom Schock überkritisch.

${ }^{3}$ Die folgenden Umformungen setzen voraus, daß alle auftretenden Größen mit Ausnahme von $B_{e}$ von Null verschieden sind.
} 
Eine Lösung der Übergangsbedingungen ist bekannt, nämlich $B_{r}=B_{l}$ und entsprechend für die anderen Größen, d.h. es gibt keinen Sprung. Diese Lösung ist nicht von Interesse und kann abgespalten werden. Es bleiben als Übergangsbedingungen

$$
0=B_{r}^{2}+\left[\alpha-1-\frac{4 \pi \rho_{l} v_{l}^{2}}{B_{l}^{2}}(\alpha-2)\right] B_{l} B_{r}+\alpha\left(\frac{8 \pi P_{l}}{B_{l}^{2}}+1\right) 4 \pi \rho_{l} v_{l}^{2}\left(1-\frac{B_{l}^{2}}{4 \pi \rho_{l} v_{l}^{2}}\right)
$$

und die letzte Gleichung im System (2.18).

\subsection{Erhalt von Gleichgewichtslösungen}

\section{Verwendetes numerisches Verfahren}

Die Gleichgewichtslösungen sind mittels einer Runge-Kutta Vorwärtsintegration, wie sie von Cash und Karp (1990) vorgeschlagen wurdef, erstellt worden.

\section{Entdimensionierung}

Die auftretenden Variablen wurden mit den in Tabelle 2.1 aufgeführten Skalen entdimensioniert.

\begin{tabular}{|l|r|}
\hline Skala & Wert \\
\hline \hline Längenskala & $100 \mathrm{~cm}$ \\
\hline Zeitskala & $1 \mathrm{sec}$ \\
\hline Massenskala & $1000 \mathrm{~g}$ \\
\hline
\end{tabular}

Tabelle 2.1: Zur Entdimensionierung des Gleichgewichtsmodells verwendete Skalen

\section{Anfangsbedingungen}

Bei allen hier vorgestellten Gleichgewichtsmodellen wurde der in Tabelle 2.2 angegebene Satz von Anfangswerten am linken Flußröhrenfußpunkt und konstanten Parametern verwendet. Der einzig verbliebene freie Startwert ist die Strömungsgeschwindigkeit im linken Fußpunkt der Röhre.

\section{Unter- und überkritische Gleichgewichtslösungen}

Diese Modelle wurden durch Vorwärtsintegration gewonnen. Der rechte Fußpunkt $s_{1}$ wird durch Iteration bestimmt; er gilt als erreicht, wenn $z\left(s_{1}\right)=z_{0}$.

\footnotetext{
${ }^{4}$ vgl. auch Press et al. (1992, S. 714ff)
} 


\begin{tabular}{|l|c|r|}
\hline Physikalische Größe & Symbol & Wert \\
\hline \hline Gravitationsbeschleunigung auf der Sonnenoberfläche & $g$ & $2.76 \cdot 10^{4} \mathrm{~cm} / \mathrm{sec}^{2}$ \\
\hline Verhältnis der spezifischen Wärmen & $\alpha$ & $5 / 3$ \\
\hline Dichte in der Flußröhre & $\rho_{0}$ & $1.58 \cdot 10^{-7} \mathrm{~g} / \mathrm{cm}^{3}$ \\
\hline Magnetische Flußdichte innerhalb der Röhre & $B_{0}$ & $1400 \mathrm{Gauss}$ \\
\hline Steigung der Flußröhre & $(d z / d s)_{0}$ & 0.7 \\
\hline Druck außerhalb der Röhre & $P_{e 0}$ & $1.16 \cdot 10^{-5} \mathrm{dyn} / \mathrm{cm}^{2}$ \\
\hline Dichte außerhalb der Röhre & $\rho_{e 0}$ & $3.03 \cdot 10^{-7} \mathrm{~g} / \mathrm{cm}^{3}$ \\
\hline Magnetische Flußdichte außerhalb der Röhre & $B_{e}$ & $0 \mathrm{Gauss}$ \\
\hline
\end{tabular}

Tabelle 2.2: Gewählte Startwerte der physikalischen Größen im linken Fußpunkt der Röhre (Größen mit Index ,0“) und verwendete konstante Parameter für alle untersuchten Gleichgewichtsmodelle. Die Werte für $P_{e 0}$ und $\rho_{e 0}$ sind Stix $(1989$, S. 47) entnommen.

\section{Transkritische Modelle ohne Schock}

Um ein transkritisches Gleichgewichtsmodell zu erhalten, wird über die zwei Parameter $v_{0}$ und $s_{1}$ iteriert. Die Strömungsgeschwindigkeit im linken Fußpunkt der Flußröhre $v_{0}$ wird so

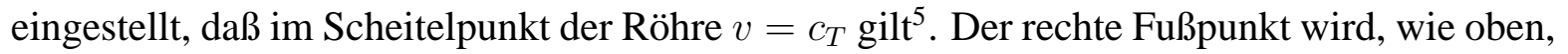
bestimmt durch die Gleichung: $z\left(s_{1}\right)=z_{0}$.

\section{Transkritische Strömungen mit Schock}

Es wird verfahren wie im Fall ohne Schock. Die Integration erfolgt aber vorerst nur bis zu der Stelle, an der der Schock eingefügt wird. D.h. an dieser Stelle werden die Übergangsbedingungen, bestehend aus Gleichung (2.19) und der sechsten Gleichung im System (2.18), erfüllt. Schließlich wird die Integration bis zum Punkt $s_{1}$ fortgeführt, der wie oben ermittelt wird. Der Ort des Schocks ist durch die Vorgabe eines Wertes einer physikalischen Variable im rechtem Röhrenfußpunkt, etwa der magnetischen Flußdichte, bestimmt.

\subsection{Ergebnisse}

Die Länge der Flußröhren in den im folgenden angegebenen Modellen ist durchweg zu klein, um als Erklärung für den Evershed-Effekt brauchbar zu sein. Das ist bei Siphonmodellen ein bekanntes Problem. Eine größere Ausdehnung kann erhalten werden, wenn im Vergleich zu dem in Tabelle 2.2 gegebenen Wert $\rho_{0}$ etwas größer gewählt oder $B_{e}>0$ eingeführt wird. Einen Überblick über die erstellten Modelle gibt Tabelle 2.3.

Es sei an dieser Stelle erwähnt, daß das Hauptanliegen dieser Arbeit die Stabilitätsanalyse von Gleichgewichtsmodellen ist. Für eine ausführlichere Diskussion von Siphonmodellen

\footnotetext{
${ }^{5}$ Nur am Scheitelpunkt kann bei dem gewählten isothermen äußeren Medium die kritische Geschwindigkeit erreicht werden (Thomas und Montesinos, 1990).
} 
verweise ich auf die Literatur (Degenhardt, 1989; Montesinos und Thomas, 1989; Degenhardt, 1990; Thomas und Montesinos, 1990; Degenhardt, 1991; Thomas und Montesinos, 1991; Degenhardt et al., 1993; Montesinos und Thomas, 1993). Es wurden Gleichgewichtsmodelle unterschiedlichen Typs erstellt:

\section{Unterkritische Modelle}

In den Abbildungen 2.1-2.3 werden ein Modell mit durchweg geringer, eines mit merklicher, aber noch deutlich unterkritischer Strömungsgeschwindigkeit und schließlich ein Modell, bei dem im Scheitelpunkt der Flußröhre die Strömungsgeschwindigkeit die kritische Geschwindigkeit beinahe erreicht, dargestellt. Mit Ausnahme der gerichteten Größen sind bei unterkritischen Gleichgewichten die Modelle symmetrisch zum Röhrenscheitelpunkt. Symmetrie ist dabei folgendermaßen zu verstehen:

- Eine Funktion $f$ wird als symmetrisch zum Scheitelpunkt $s_{\text {Scheitel }}$ bezeichnet, wenn sie spiegelsymmetrisch zu einer Achse parallel zur Ordinate durch den Abszissenwert $s_{\text {Scheitel }}$ ist, d.h. $f(s)=f\left(2 s_{\text {Scheitel }}-s\right)$.

Die Beträge der vektoriellen Größen sind ebenfalls symmetrisch zum Scheitelpunkt. Mit steigender Höhe über der Sonnenoberfläche nehmen Druck und Dichte inner- wie außerhalb der Röhre ab. Der Betrag der magnetischen Flußdichte muß nicht notwendigerweise mit zunehmender Höhe kleiner werden, in Abhängigkeit der Anfangswerte können sich Minima bei kleinerer Höhe als der des Scheitelpunkts befinden. Thomas (1988) gibt diesen Minima den Namen ,bulge points“, weil an diesen Stellen der Röhrenquerschnitt ${ }^{6}$ ein Maximum aufweist. Bei den in dieser Arbeit dargestellten unterkritischen Modellen fallen diese Punkte auf den Röhrenscheitelpunkt. Eine Modellserie mit $B_{0}=1200$ Gauss zeigt vom Röhrenscheitelpunkt deutlich verschiedene Punkte größten Röhrenquerschnitts. Der Verlauf der Strömungsgeschwindigkeit ergibt sich als Folge daraus, daß die Größe $\rho v / B$ nicht von der Bogenlänge abhängt. Der Geschwindigkeitszuwachs mit wachsender Höhe über der Sonnenoberfläche liegt bei allen betrachteten unterkritischen Modellen im Bereich von weniger als einer Größenordnung. Bei den in dieser Arbeit angegebenen Gleichgewichten ist die kritische Geschwindigkeit im gesamten Verlauf der Flußröhre etwa gleich der Schallgeschwindigkeit.

\section{Transkritische Modelle}

In Abbildung 2.4 ist ein transkritisches Modell ohne Schock dargestellt. Dieses Modell ist nicht symmetrisch zum Röhrenscheitelpunkt. Links vom Scheitel ist die Strömung unter-, rechts überkritisch. Für den unterkritischen Teil lassen sich die Betrachtungen aus dem vorangegangenen Abschnitt übertragen. Bei den transkritischen Modellen liegen „,bulge point“ und Scheitelpunkt nicht am gleichen Ort. Im überkritischen Ast fallen Druck und Dichte in

\footnotetext{
${ }^{6}$ Aufgrund der Erhaltung des magnetischen Flusses und der Magnetfeldrichtung entlang der Röhre gilt $A \sim$ $1 / B, A$ bezeichnet den Röhrenquerschnitt.
} 


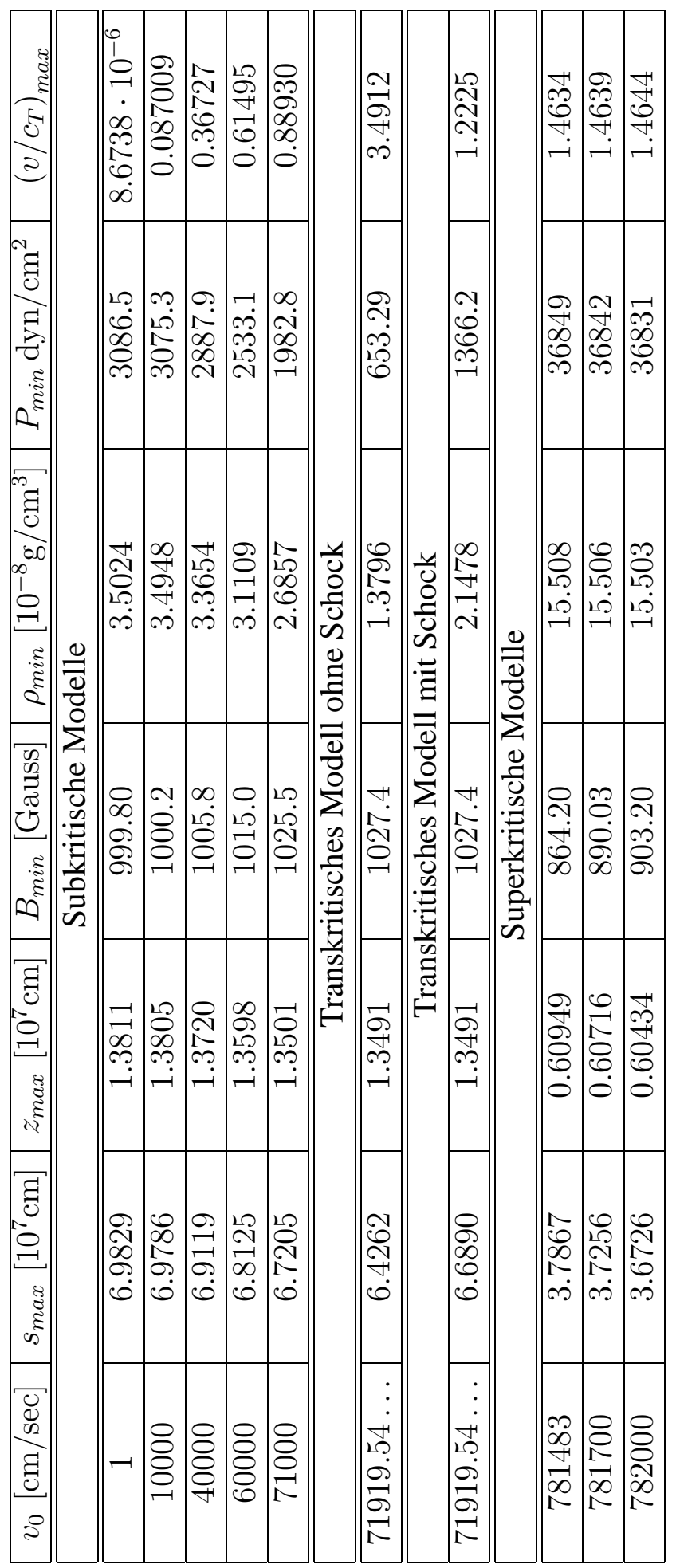

Tabelle 2.3: Charakteristische Größen aus verschiedenen Gleichgewichtsmodellen 
der Röhre im Bereich des rechten Röhrenfußpunkts auf so kleine Werte ab, daß bezweifelt werden muß, ob man Strömungen dieser Art auf der Sonne vorfinden kann. Der Wert für die magnetische Flußdichte liegt hingegen durchaus noch im Bereich dessen, was Messungen ergeben.

Transkritische Modelle müssen nicht zwangsläufig zu unrealistisch niedrigen Werten von beobachtbaren Größen im rechten Fußpunkt der Flußröhre führen. Bei einem Schock in dem überkritischen Teil einer durchströmten, dünnen magnetischen Flußröhre ändern sich innerhalb eines sehr kleinen Bereichs die physikalischen Größen nach folgendem Muster (FerrizMas und Moreno-Insertis, 1987)

- $P_{l}<P_{r}$

- $\rho_{l}<\rho_{r}$

- $v_{l}>v_{r}$ mit $v_{l}>c_{T, l}$ und $v_{r}<c_{T, r} . c_{T, l}$ bzw. $c_{T, r}$ meinen die kritische Geschwindigkeit links bzw. rechts vom Schock.

- $B_{l}>B_{r}$

Man hat nach dem Durchlaufen des Schocks wieder eine unterkritische Strömung, auf die dann die oben genannten Betrachtungen zutreffen. Die Werte von Druck und Dichte am rechten Röhrenfußpunkt liegen bei solchen Modellen in einem plausiblen Rahmen. Ein transkritisches Modell mit Schock findet sich in Abbildung 2.5.

\section{Superkritische Modelle}

Bei rein überkritischen Modellen fällt die Strömungsgeschwindigkeit mit wachsender Höhe über der Sonnenoberfläche bei ebenfalls kleiner werdender magnetischer Flußdichte, was einem anwachsenden Röhrenquerschnitt entspricht. Dieses Verhalten ist aus der Hydrodynamik von Überschallströmungen bekannt. Bei überkritischen Modellen gilt das gleiche Symmetrieverhalten, wie bei den unterkritischen. Für Strömungen in Sonnenfleckenpenumbren sind rein überkritische Modelle bedeutungslos. 

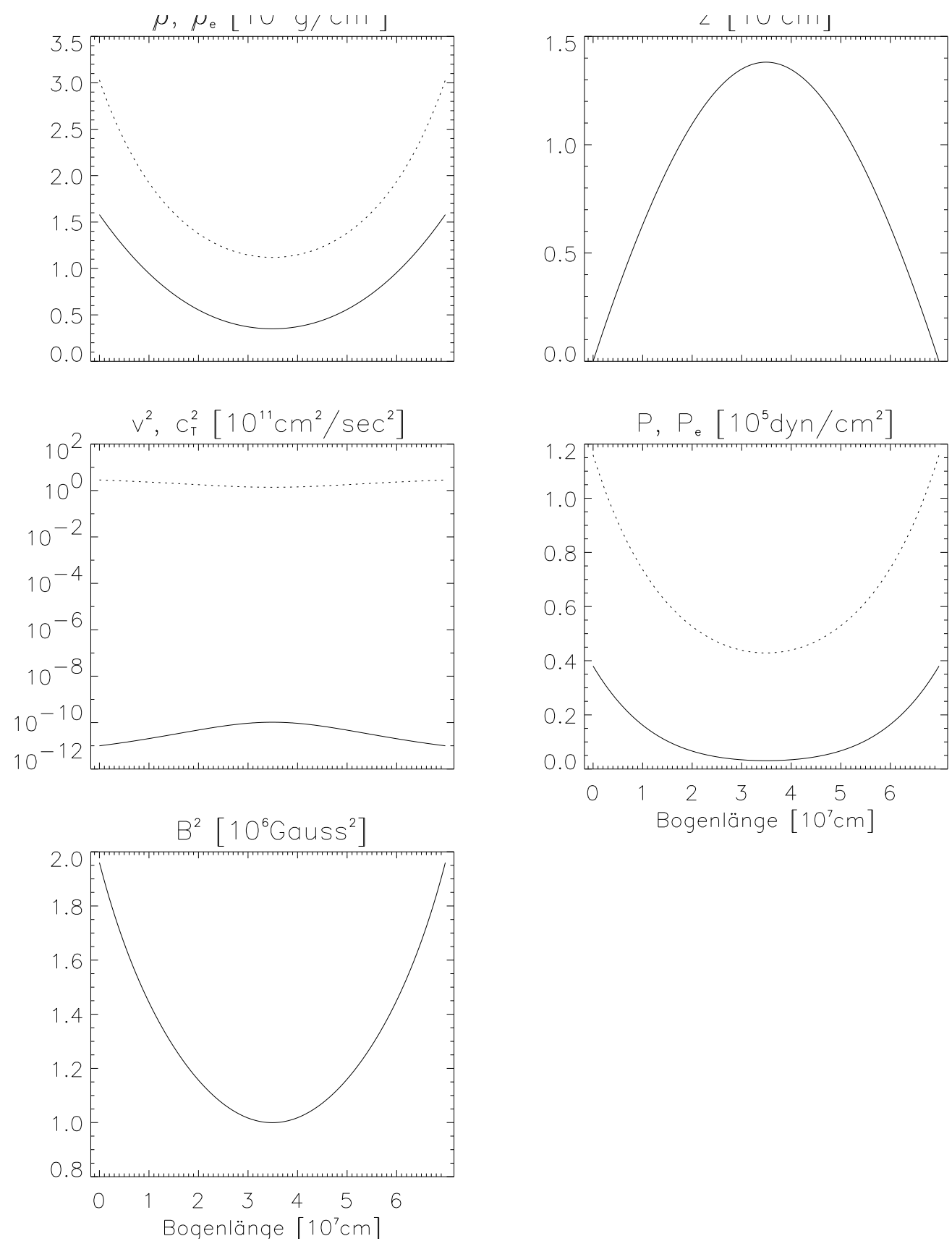

Abbildung 2.1: Gleichgewichtsmodell einer durchströmten dünnen magnetischen Flußröhre mit überall geringer Strömungsgeschwindigkeit. Die Strömungsgeschwindigkeit im linken Röhrenfußpunkt beträgt $1 \mathrm{~cm} / \mathrm{sec}$. Die Größen im umgebenden Medium und die kritische Geschwindigkeit sind, wie auch in den folgenden Gleichgewichtsdarstellungen, jeweils durch punktierte Linien repräsentiert. 

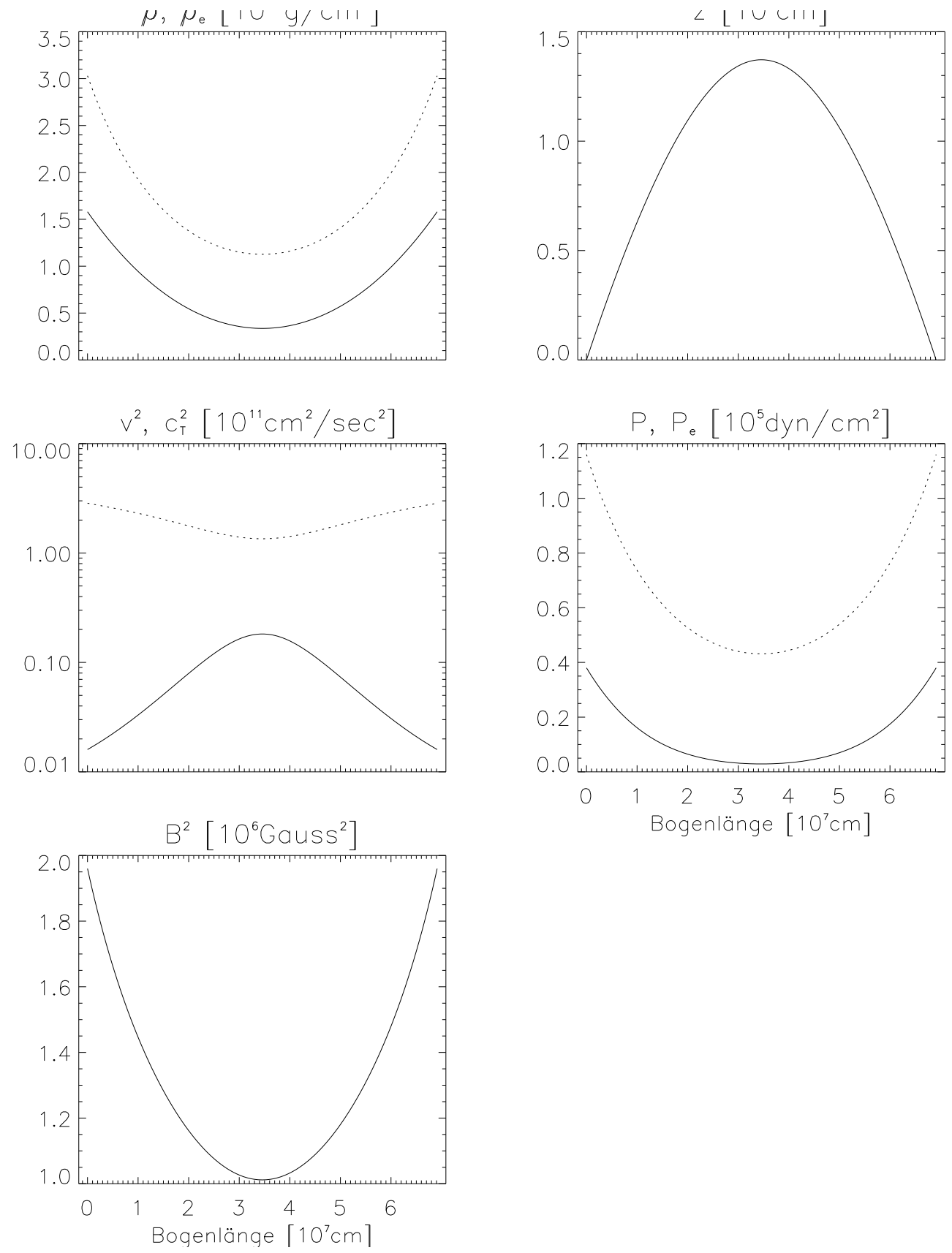

Abbildung 2.2: Gleichgewichtsmodell mit einer Strömungsgeschwindigkeit von $40000 \mathrm{~cm} / \mathrm{sec}$ im linken Fußpunkt. Der wesentliche Unterschied zu Abbildung 2.1 liegt in der deutlich geringeren Differenz von kritischer und Strömungsgeschwindigkeit. 

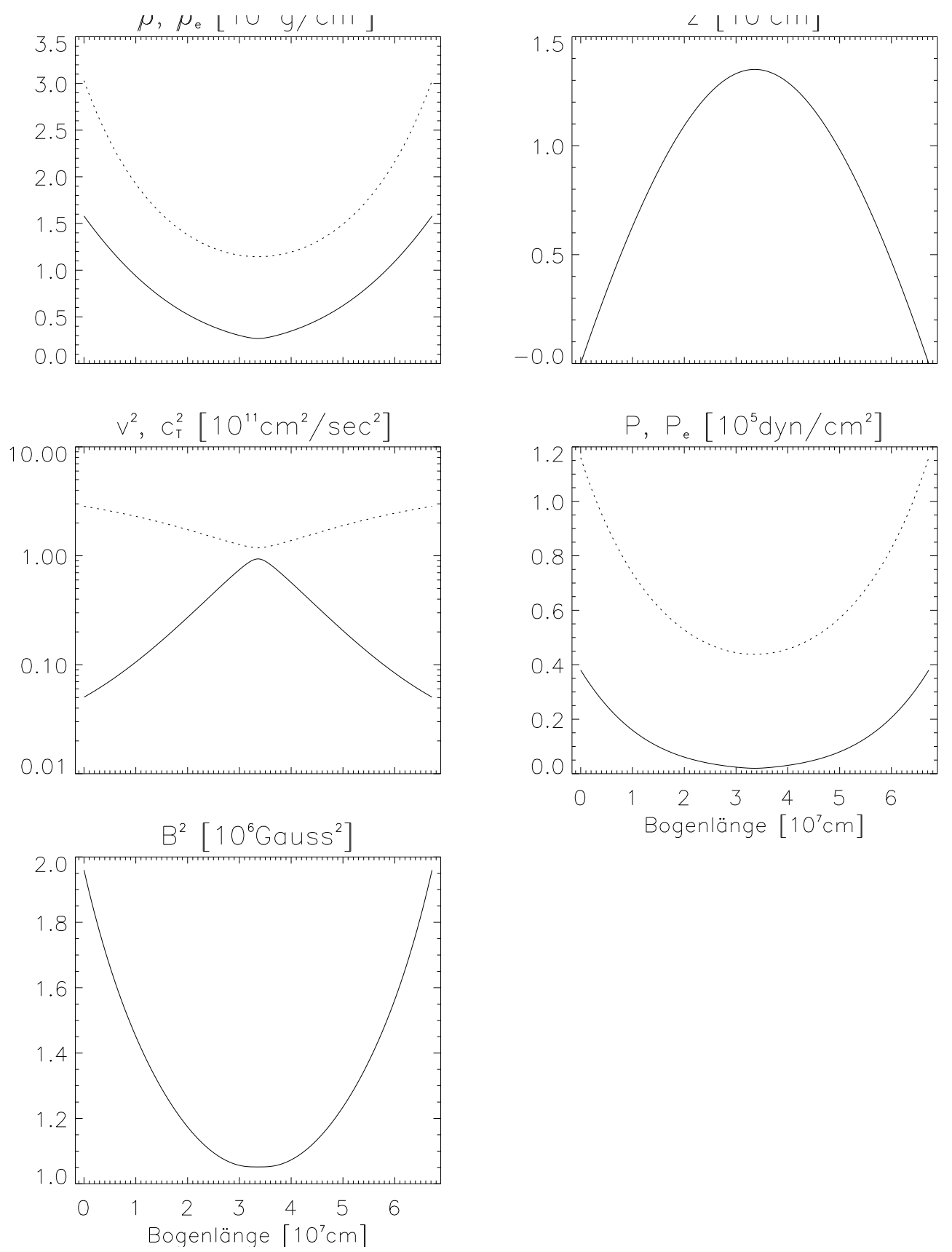

Abbildung 2.3: Gleichgewichtsmodell mit einer Strömungsgeschwindigkeit von $71000 \mathrm{~cm} / \mathrm{sec}$ im linken Fußpunkt. Die Differenz von kritischer und Strömungsgeschwindigkeit ist noch geringer als in Abbildung 3.2. 

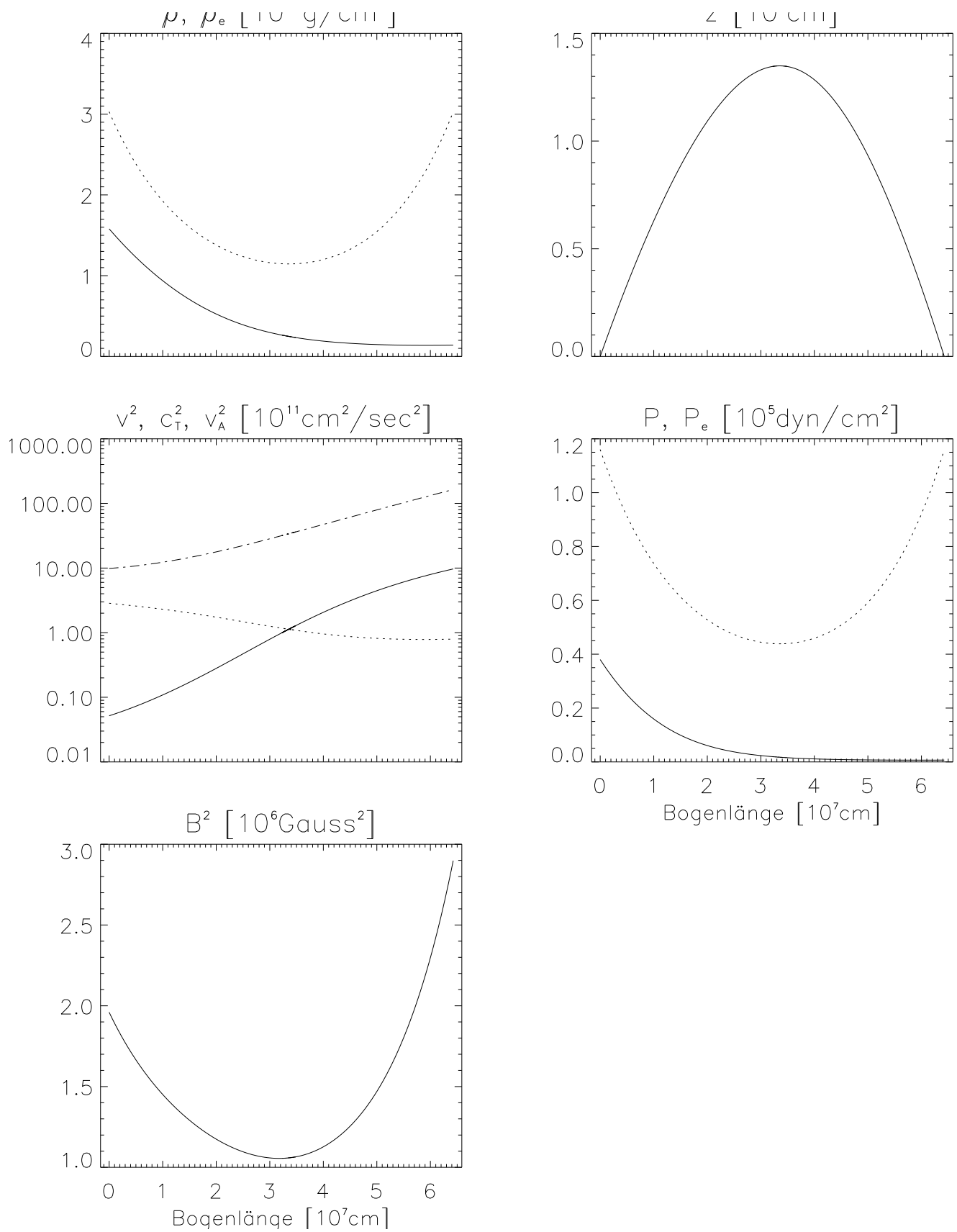

Abbildung 2.4: Transkritisches Gleichgewichtsmodell ohne Schock. Die Strömungsgeschwindigkeit im linken Fußpunkt ist $71919.5498252681 \mathrm{~cm} / \mathrm{sec}$. Diese Angabe ist nur im Rahmen der numerischen Genauigkeit vertrauenswürdig, da eine transkritische Strömung bei den in Tabelle 2.2 genannten Startwerten für genau einen Startwert der Strömungsgeschwindigkeit im linken Fußpunkt existiert. Hier und bei allen folgenden Modellen mit teilweise überkritischer Strömungsgeschwindigkeit ist bei den Geschwindigkeiten zusätzlich die Alfvéngeschwindigkeit mit einer strich-punktierten Linie dargestellt. 

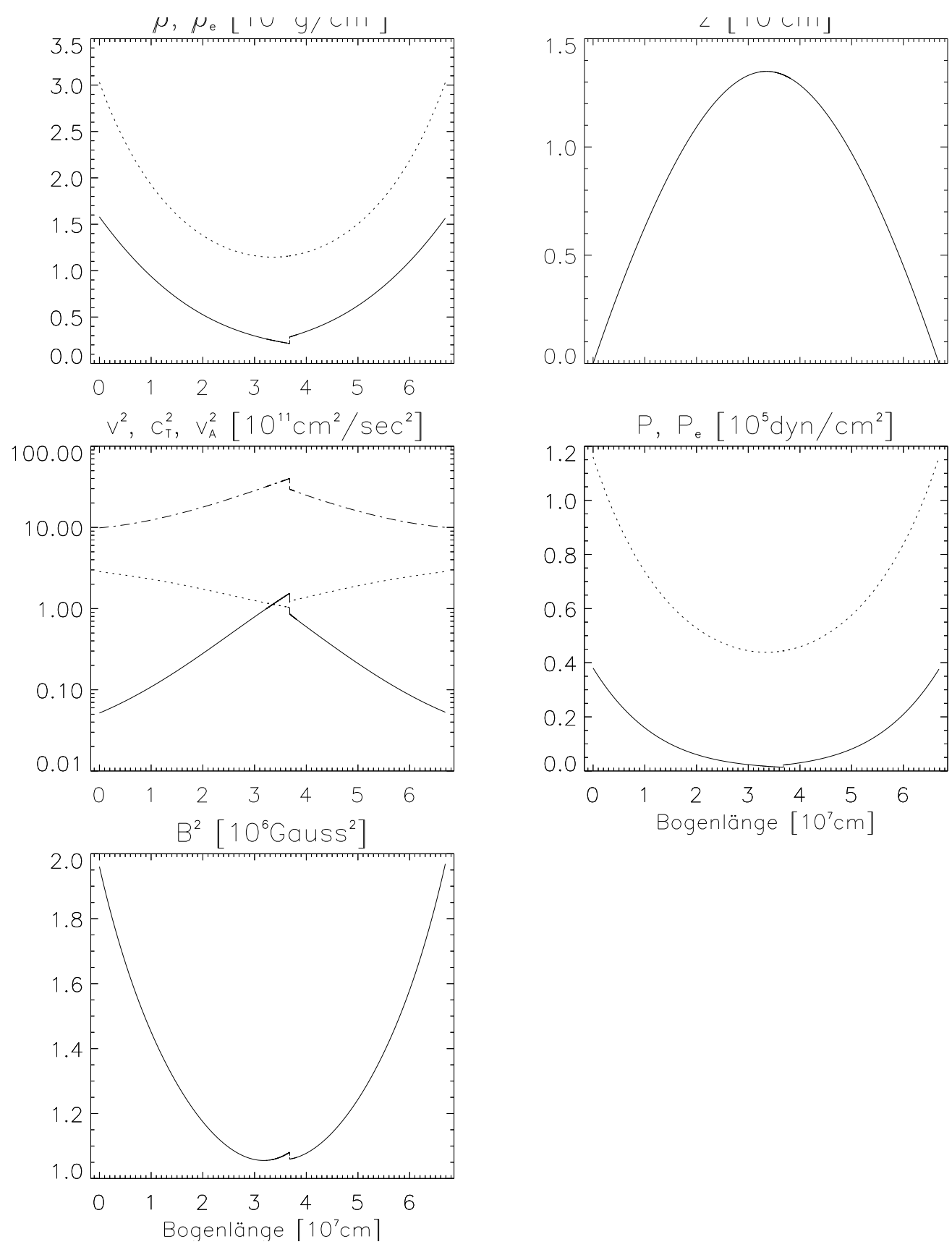

Abbildung 2.5: Transkritisches Gleichgewichtsmodell mit Schock. Startwerte wie in Abb. 2.4. Der Ort des Schocks liegt recht nahe am Röhrenscheitelpunkt, um die Sprünge in den beobachtbaren Größen klein zu halten. 

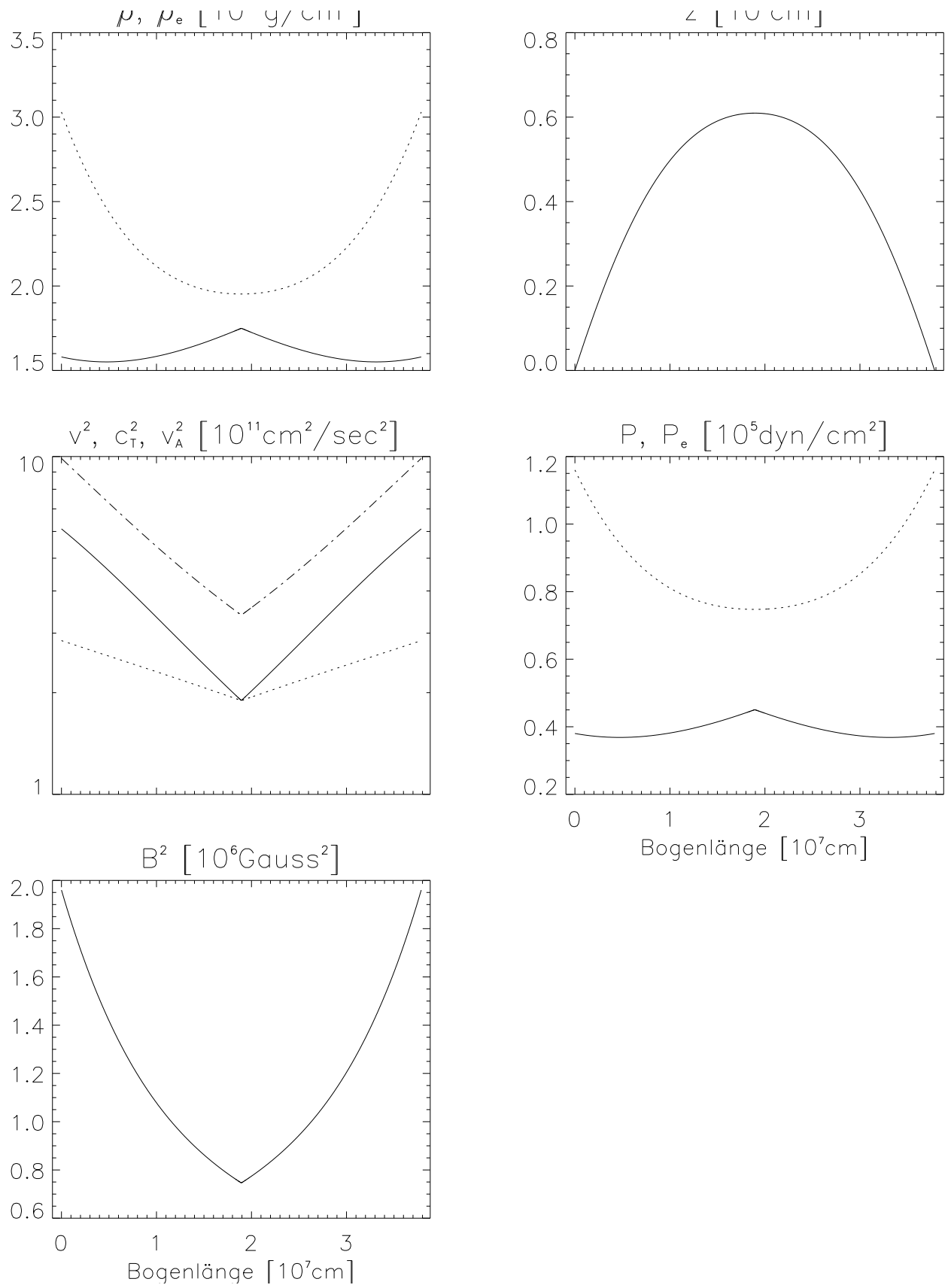

Abbildung 2.6: Superkritische Strömung. Die Strömungsgeschwindigkeit im linken Fußpunkt der Flußröhre beträgt $781483 \mathrm{~cm} / \mathrm{sec}$. Im Vergleich mit dem in Abbildung 2.7 dargestellten überkritischen Gleichgewicht ist ersichtlich, daß sich mit zunehmender Annäherung von Strömungs- an kritische Geschwindigkeit am Scheitelpunkt ein Knick der physikalischen Größen in der Flußröhre ergibt. Bei dem abgebildeten Gleichgewicht sind jedoch alle Größen stetig differenzierbar. 

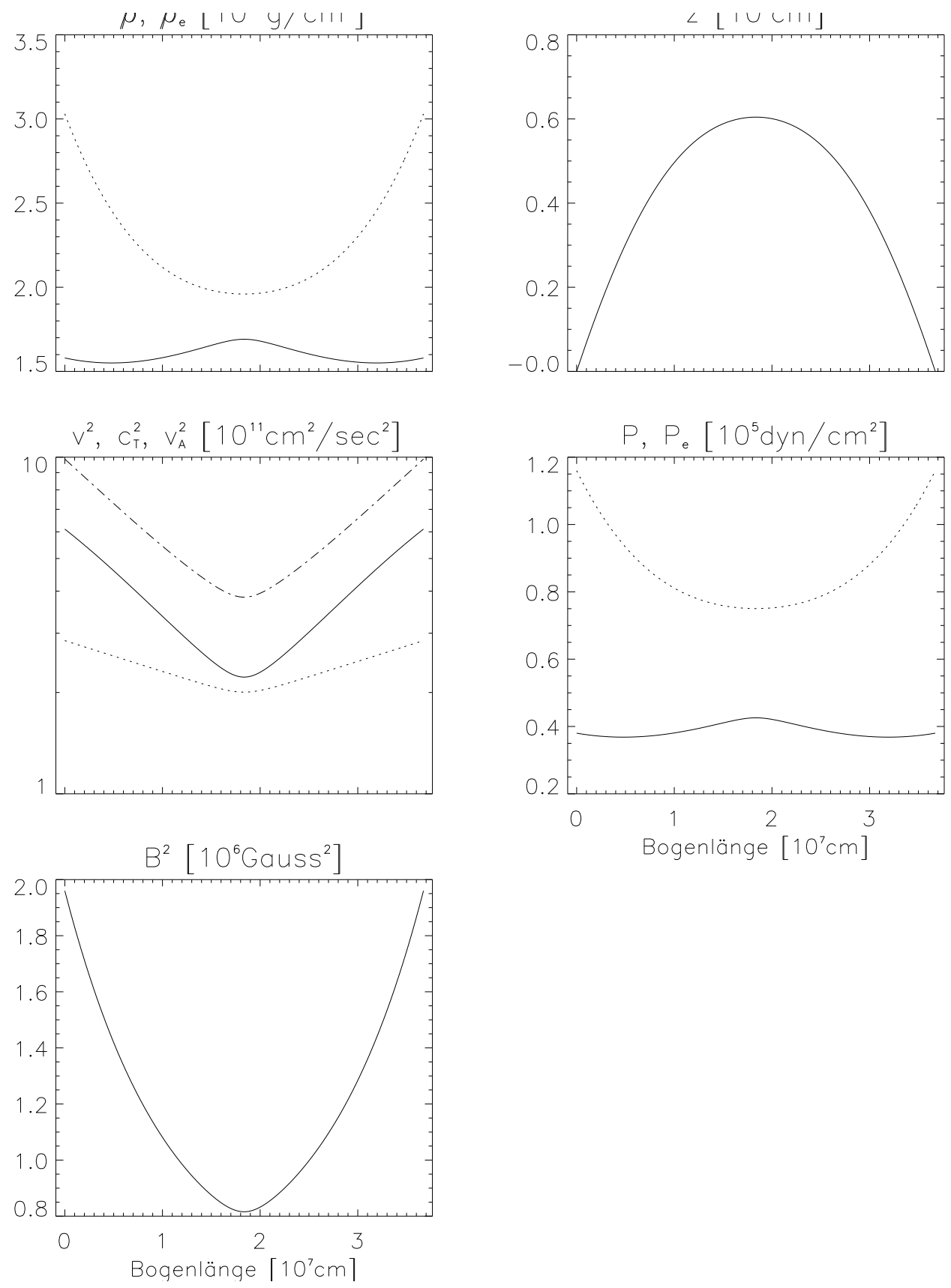

Abbildung 2.7: Superkritische Strömung. Die Strömungsgeschwindigkeit im linken Fußpunkt der Flußröhre beträgt $782000 \mathrm{~cm} / \mathrm{sec}$. Solange die Strömungsgeschwindigkeit überall kleiner als die Alfvéngeschwindigkeit bleibt, werden die Kurven bei wachsender Strömungsgeschwindigkeit im linken Fußpunkt glatter. 


\section{Stabilitätsanalyse}

Dieser Arbeit liegt der von Schmitt (1995; 1998) abgeleitete Formalismus zugrunde, der eine lineare Analyse darstellt. In einer solchen wird jede physikalische Veränderliche als Summe aus Gleichgewichtsgröße und einer infinitesimal kleinen, zeitabhängigen Störung aufgefaßt. Dies sei am Beispiel der Höhe der Flußröhre über der Sonnenoberfläche $z(s, t)$ erläutert.

$$
z(s, t)=z(s)+z_{s}(s, t)
$$

$z(s)$ ist die nur von der ungestörten Bogenlänge $s$ abhängige Gleichgewichtsgröße, $z_{s}(s, t)$ die darüber hinaus von der Zeit $t$ abhängige Störung. Störgrößen werden mit dem Index , $s$ “ gekennzeichnet. Terme höherer als erster Ordnung in den Störgrößen werden in einer linearen Analyse vernachlässigt.

\subsection{Störgrößen}

Die Störung des Orts der Flußröhre ist!

$$
\vec{\xi}(s, t)=\eta(s, t) \hat{t}+\epsilon(s, t) \hat{n}+\zeta(s, t) \hat{b}
$$

$\hat{t}, \hat{n}$ sowie $\hat{b}$ stellen das aus Abschnitt 2 bekannte begleitende Dreibein der ungestörten Flußröhre dar. Im weiteren werden Orts- und Zeitabhängigkeit von $\vec{\xi}, \eta, \epsilon$ und $\zeta$ nicht mehr mit angegeben.

Alle anderen Störungen erhält man nach Schmitt (1995); 1998) aus $\vec{\xi}$ mittels der Gleichun-

\footnotetext{
${ }^{1}$ Alle im folgenden angegebenen Störgrößen sind Lagrangescher Natur. Allerdings wird, wie auch bei Frieman und Rotenberg (1960), als unabhängige Ortsvariable anstelle des Ortes, an dem sich das Flüssigkeitselement zu einer bestimmten Zeit befand, die Gleichgewichtsbogenlänge $s$ gewählt. Als Folge dieser Wahl sind die Koeffizienten im Gleichungssystem (3.1) nicht von der Zeit abhängig, und man kann die Zeitabhängigkeit von (3.1) mittels eines Exponentialansatzes behandeln.
} 
gen

$$
\begin{aligned}
z_{s} & =\frac{d z}{d s} \eta+R_{C} \frac{d^{2} z}{d s^{2}} \epsilon \\
B_{s} & =B\left[\frac{c_{T}^{2}}{v_{A}^{2}}\left(\frac{\partial \eta}{\partial s}-\frac{\epsilon}{R_{C}}\right)-\frac{\rho_{e}}{\rho} \frac{g z_{s}}{c_{S}^{2}+v_{A}^{2}}\right] \\
\rho_{s} & =-\rho\left[\frac{c_{T}^{2}}{v_{A}^{2}}\left(\frac{\partial \eta}{\partial s}-\frac{\epsilon}{R_{C}}\right)+\frac{\rho_{e}}{\rho} \frac{g z_{s}}{c_{S}^{2}+v_{A}^{2}}\right] \\
P_{s} & =-\rho_{e} g z_{s}-\frac{B B_{s}}{4 \pi} \\
v_{s} \cdot \hat{t} & =\frac{\partial \eta}{\partial t}+v\left(\frac{\partial \eta}{\partial s}-\frac{\epsilon}{R_{C}}\right) \\
v_{s} \cdot \hat{n} & =\frac{\partial \epsilon}{\partial t}+v\left(\frac{\partial \epsilon}{\partial s}+\frac{\eta}{R_{C}}\right) \\
v_{s} \cdot \hat{b} & =\frac{\partial \zeta}{\partial t}+v \frac{\partial \zeta}{\partial s}
\end{aligned}
$$

Betrag der Gravitationsbeschleunigung auf der Sonne $g$, Höhe über der Sonnenoberfläche $z$, Krümmungsradius $R_{c}$, Betrag der magnetischen Flußdichte $B$, kritische Geschwindigkeit $c_{T}$, Alfvéngeschwindigkeit $v_{A}$, Dichte des umgebenden Mediums $\rho_{e}$, Dichte $\rho$ und Strömungsgeschwindigkeit $v$ sind aus den Gleichgewichtsmodellen bekannt.

\subsection{Das beschreibende Gleichungssystem}

Der Vektor $\vec{\xi}$ wird durch folgendes Gleichungssystem beschrieben?

$$
\begin{aligned}
\mathcal{P} \frac{\partial^{2} \eta}{\partial t^{2}}+2 \mathcal{Q}\left(\frac{\partial^{2} \eta}{\partial s \partial t}-\frac{1}{R_{C}} \frac{\partial \epsilon}{\partial t}\right)-\mathcal{A} \epsilon-\mathcal{B} \frac{\partial \epsilon}{\partial s}-\mathcal{C} \eta-\frac{\partial \mathcal{E}}{\partial s} \frac{\partial \eta}{\partial s}-\mathcal{E} \frac{\partial^{2} \eta}{\partial s^{2}}=0 \\
\mu \mathcal{P} \frac{\partial^{2} \epsilon}{\partial t^{2}}+2 \mathcal{Q}\left(\frac{\partial^{2} \epsilon}{\partial s \partial t}+\frac{1}{R_{C}} \frac{\partial \eta}{\partial t}\right)-\mathcal{A} \eta+\frac{\partial \mathcal{B}}{\partial s} \eta+\mathcal{B} \frac{\partial \eta}{\partial s}-\mathcal{H} \epsilon-\frac{\partial \mathcal{J}}{\partial s} \frac{\partial \epsilon}{\partial s}-\mathcal{J} \frac{\partial^{2} \epsilon}{\partial s^{2}}=0 \\
\mu \mathcal{P} \frac{\partial^{2} \zeta}{\partial t^{2}}+2 \mathcal{Q} \frac{\partial^{2} \zeta}{\partial s \partial t}-\frac{\partial \mathcal{J}}{\partial s} \frac{\partial \zeta}{\partial s}-\mathcal{J} \frac{\partial^{2} \zeta}{\partial s^{2}}=0
\end{aligned}
$$

$\mu$ dient zur Parametrisierung der infolge der Bewegung der Flußröhre durch das umgebene Medium modifizierten Trägheit. Bisher gibt es in der Literatur noch keine konsistente Beschreibung dieser Größe. Nach Moreno-Insertis et al. (1996) läßt sie sich nicht im Rahmen der Näherung dünner Flußröhren beschreiben. In dieser Arbeit wird, Schmitt (1995; 1998) folgend, $\mu=1$ gesetzt.

\footnotetext{
${ }^{2}$ Gleichungen (4.25)-(4.27) in Schmitt (1998)
} 


\section{Koeffizienten}

Die Koeffizienten $\mathcal{P}, \mathcal{Q}, \mathcal{A}-\mathcal{C}, \mathcal{E}, \mathcal{H}$ und $\mathcal{J}$ im Gleichungssystem (3.1) enthalten nur Gleichgewichtsgrößen und sind damit nicht von der Zeit abhängig; sie sind definiert als

$$
\begin{aligned}
\mathcal{P}= & \rho A \\
\mathcal{Q}= & \mathcal{P} v \\
\mathcal{A}= & -\frac{d}{d s}\left(\frac{\mathcal{E}}{R_{C}}\right)+\mathcal{P} \varpi \vec{g} \cdot \hat{n} \\
\mathcal{B}= & -\frac{\mathcal{E}}{R_{C}}+\mathcal{P} \nu \vec{g} \cdot \hat{n} \\
\mathcal{C}= & \mathcal{P}\left(\varpi \vec{g} \cdot \hat{t}+\frac{\nu \vec{g} \cdot \hat{n}}{R_{C}}\right) \\
\mathcal{E}= & \mathcal{P}\left(c_{T}^{2}-v^{2}\right) \\
\mathcal{H}= & \frac{\mathcal{P}}{c_{S}^{2}+v_{A}^{2}}\left[\frac{c_{S}^{2} v^{2}-c_{S}^{2} v_{A}^{2}+v_{A}^{2}}{R_{C}^{2}}+\frac{\rho_{e} / \rho\left(2 v_{A}^{2}-v^{2}\right)+v_{A}^{2}}{R_{C}} \cdot \hat{n}+\right. \\
& \left.+\frac{\rho_{e}}{\rho}(\vec{g} \cdot \hat{n})^{2}\left(1-H_{\rho e}^{-1} \frac{c_{S}^{2}+v_{A}^{2}}{g}\right)\right] \\
\mathcal{J}= & \mathcal{P}\left(v_{A}^{2}-v^{2}\right)
\end{aligned}
$$

mit den Hilfsvariablen

$$
\begin{aligned}
\varpi & =\frac{1}{\rho}\left[\left(\vec{g} \cdot \hat{t}-\frac{1}{2} \frac{\partial v^{2}}{\partial s}\right) \frac{\rho_{e}}{c_{S}^{2}+v_{A}^{2}}-\frac{\partial}{\partial s}\left(\frac{c_{T}^{2}}{v_{A}^{2}} \rho_{e}\right)\right] \\
\nu & =1-\frac{c_{T}^{2}}{v_{A}^{2}} \frac{\rho_{e}}{\rho}
\end{aligned}
$$

$A$ ist die Querschnittsfläche der Flußröhre, ihr Wert am linken Fußpunkt der Röhre wurde bei allen Rechnungen zur Stabilitätsanalyse als $A_{0}=\pi \cdot\left(2.5 \cdot 10^{6} \mathrm{~cm}\right)^{2}$ gewählt. Die äußere Dichteskalenhöhe ist gegeben durch

$$
H_{\rho e}^{-1}=-\frac{d \ln \rho_{e}}{d z}
$$

${ }^{3}$ vgl. Gleichungen (4.28)-(4.35) bei Schmitt (1998) 
Die in den Parameterdefinitionen (3.2) auftretenden Ableitungen der Geschwindigkeiten folgen aus den Gleichungen (2.15), (2.16) und (2.12); sie sind gegeben durch

$$
\begin{aligned}
\frac{d c_{S}^{2}}{d s} & =\frac{\alpha-1}{\rho} c_{S}^{2} \frac{d \rho}{d s} \\
\frac{d v_{A}^{2}}{d s} & =-\frac{1}{\rho}\left[\left(v_{A}^{2}+2 c_{S}^{2}\right) \frac{d \rho}{d s}+2 g \rho_{e} \frac{d z}{d s}\right] \\
\frac{d c_{T}^{2}}{d s} & =\frac{d v_{A}^{2} / d s c_{S}^{4}+v_{A}^{4} d c_{S}^{2} / d s}{\left(v_{A}^{2}+c_{S}^{2}\right)^{2}}
\end{aligned}
$$

Die Ableitung des Krümmungsradius' ist

$$
\begin{aligned}
\frac{d R_{C}}{d s}= & -\frac{1}{R_{C} d^{2} z / d s^{2}}\left(\frac{d z}{d s}+R_{C}^{2} \frac{d^{3} z}{d s^{3}}\right) \\
\frac{d^{3} z}{d s^{3}}= & \frac{g}{v^{2}-v_{A}^{2}}\left\{\frac{1}{\rho}\left(\frac{d \rho_{e}}{d s}-\frac{\rho_{e}}{\rho} \frac{d \rho}{d s}\right)\left[1-\left(\frac{d z}{d s}\right)^{2}\right]-\left(\frac{\rho_{e}}{\rho}-1\right) 2 \frac{d z}{d s} \frac{d^{2} z}{d s^{2}}-\right. \\
& \left.-\frac{\left(\rho_{e} / \rho-1\right)}{v^{2}-v_{A}^{2}}\left[1-\left(\frac{d z}{d s}\right)^{2}\right]\left(\frac{d v^{2}}{d s}-\frac{d v_{A}^{2}}{d s}\right)\right\}
\end{aligned}
$$

\section{Symmetrieeigenschaften der Koeffizienten bei rein unter- bzw. überkritischen Gleichgewichten}

Legt man die Symmetrie zum Röhrenscheitelpunkt der rein unter- und überkritischen Gleichgewichtsmodelle zugrunde, so lassen sich aus den Gleichungen (3.2) Symmetrien der Koeffizienten im Gleichungssytem (3.1) ableiten. Dabei ist zu beachten, daß die Ableitung einer symmetrischen Funktion nach der Bogenlänge eine antisymmetrische Funktion ist. Antisymmetrisch ist hierbei so zu verstehen:

- Eine Funktion $f$ ist antisymmetrisch zum Röhrenscheitelpunkt $s_{\text {Scheitel }}$, wenn sie punktsymmetrisch zum Abszissenwert $s_{\text {Scheitel }}$ ist, d.h. $f(s)=-f\left(2 s_{\text {Scheitel }}-s\right)$.

Aufgrund der Gleichungen (2.5) ist $\vec{g} \cdot \hat{t}$ antisymmetrisch und, da $R_{C}$ mit Gleichung im System (2.4) symmetrisch ist, $\vec{g} \cdot \hat{n}$ symmetrisch. Des weiteren ist die Größe $\varpi$ antisymmetrisch, während $\nu$ und $H_{\rho e}^{-1}$ symmetrisch sind. Man findet

- Symmetrie zum Scheitelpunkt bei den Koeffizienten $\mathcal{P}, \mathcal{Q}, \mathcal{B}, \mathcal{C}, \mathcal{H}, \mathcal{E}$ und $\mathcal{J}$,

- Antisymmetrie bei $\mathcal{A}, \partial \mathcal{B} / \partial s, \partial \mathcal{E} / \partial s$ und $\partial \mathcal{J} / \partial s$.

In den Abbildungen 3.1-3.3 sind die Koeffizienten des Störgleichungssystems (3.1) zu drei unterkritischen Modellen graphisch dargestellt, in den Abbildungen 3.4 und 3.5 die für die transkritischen und in den Abbildungen 3.6 und 3.7 diejenigen für zwei überkritische Gleichgewichtsmodelle. 

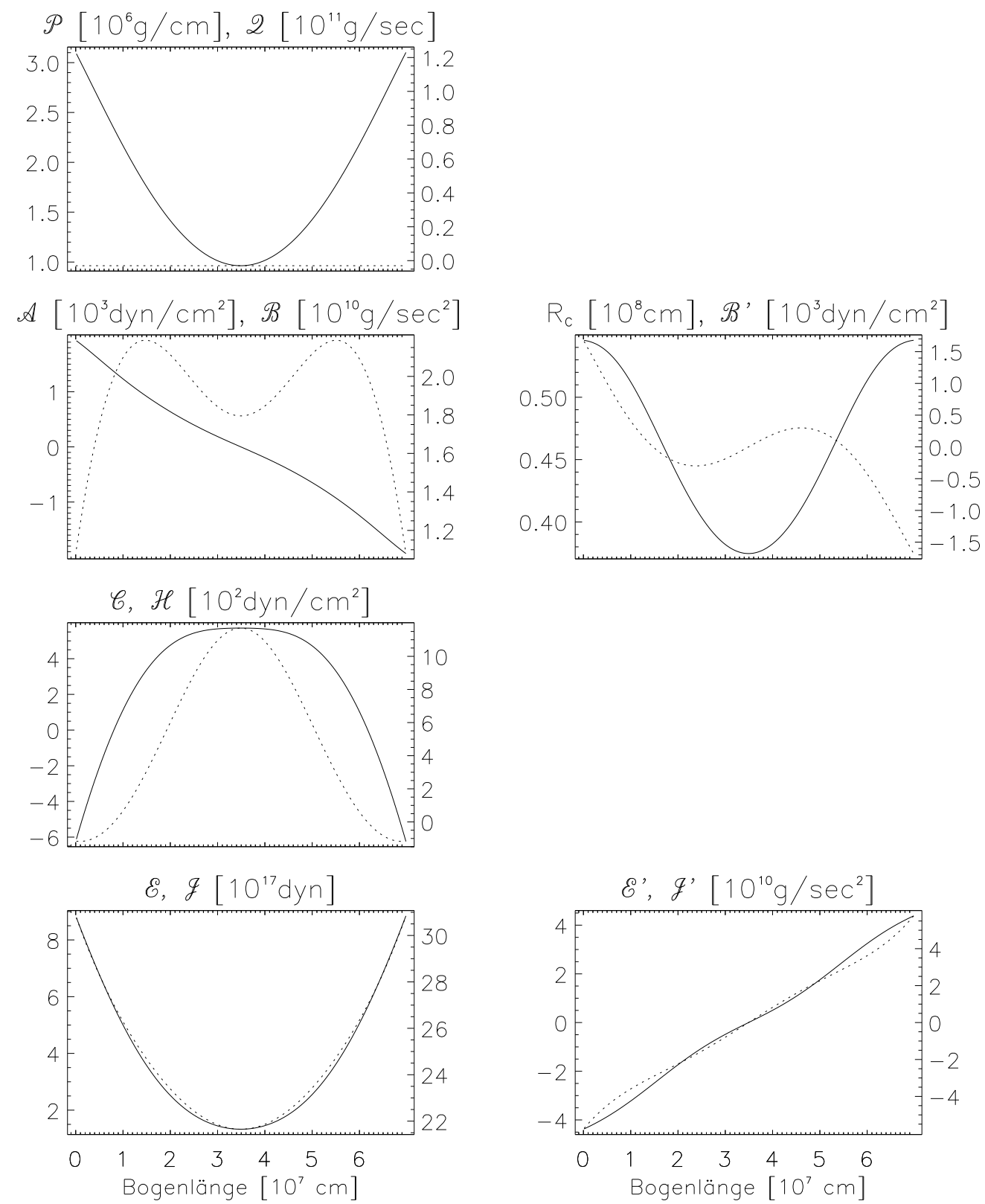

Abbildung 3.1: Koeffizienten im Gleichungssystem (3.1) für das in Abbildung 2.1 gezeigte, unterkritische Gleichgewicht. Zu der jeweils links in der Überschrift stehenden Größe gehört hier und bei den folgenden graphischen Darstellungen der Koeffizienten die durchgezogene Linie und die linke Ordinate. Die gepunktete Linie stellt jeweils die Größe rechts in der Überschrift dar, der Wert ist der rechten Ordinate zu entnehmen. Die Strömungsgeschwindigkeit im linken Fußpunkt der Röhre ist $1 \mathrm{~cm} / \mathrm{sec}$. 

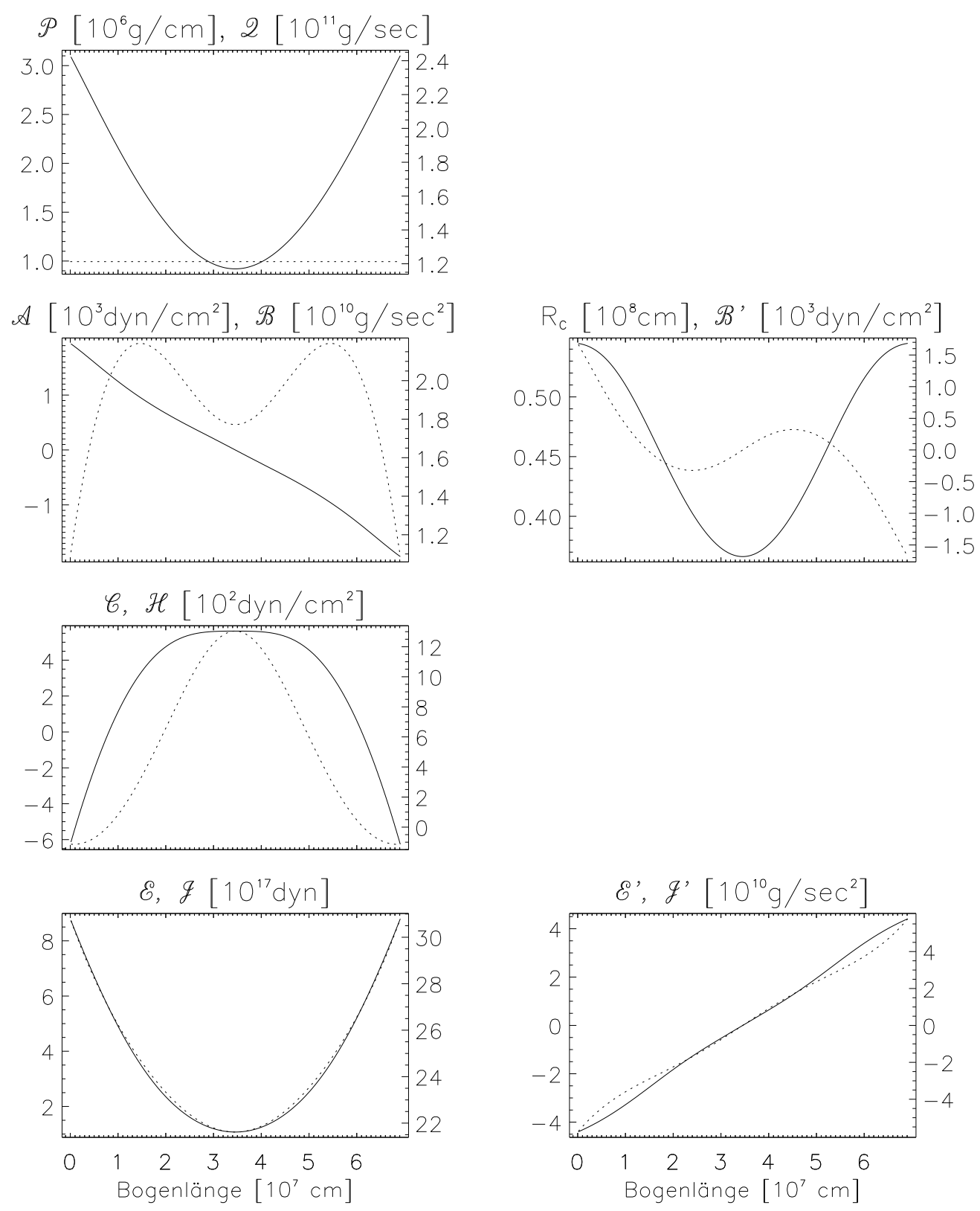

Abbildung 3.2: Koeffizienten zum Gleichungssystem (3.1) für das in Abbildung 2.2 dargestellte, unterkritische Gleichgewichtsmodell. Bei diesem Modell beträgt die Strömungsgeschwindigkeit im linken Fußpunkt der Flußröhre $40000 \mathrm{~cm} / \mathrm{sec}$. Der Unterschied zu den Koeffizienten aus Abbildung 3.1 ist gering, einzig der Wert von $\mathcal{Q}$ unterscheidet sich deutlich. 

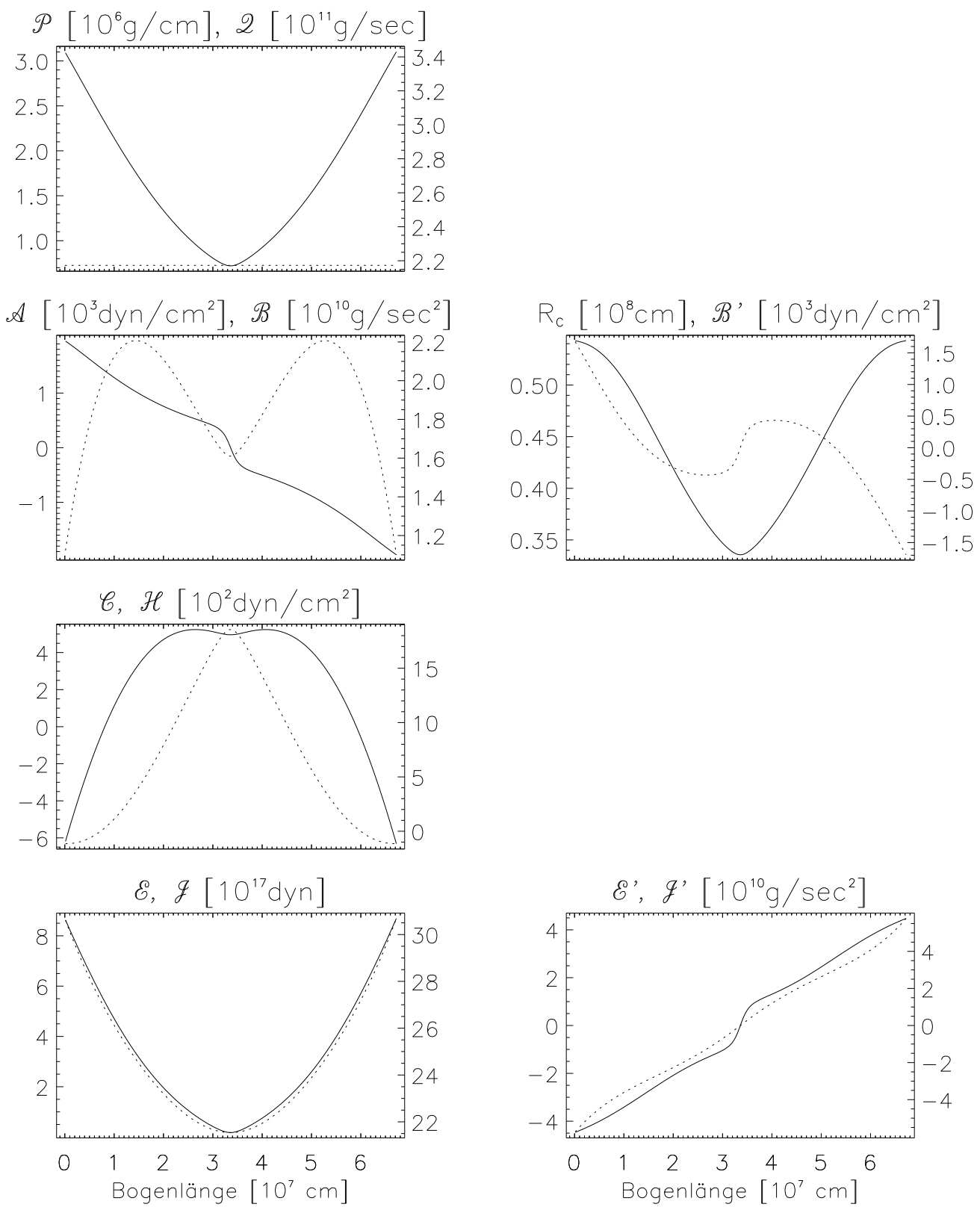

Abbildung 3.3: Koeffizienten zum Gleichungssystem (3.1) für das unterkritische Gleichgewichtsmodell aus Abbildung 2.3 mit einer Strömungsgeschwindigkeit im linken Röhrenfußpunkt von $71000 \mathrm{~cm} / \mathrm{sec}$. Im Vergleich zu den schwächer durchströmten Gleichgewichten fallen die Verläufe in der Umgebung des Röhrenscheitelpunkts auf. 

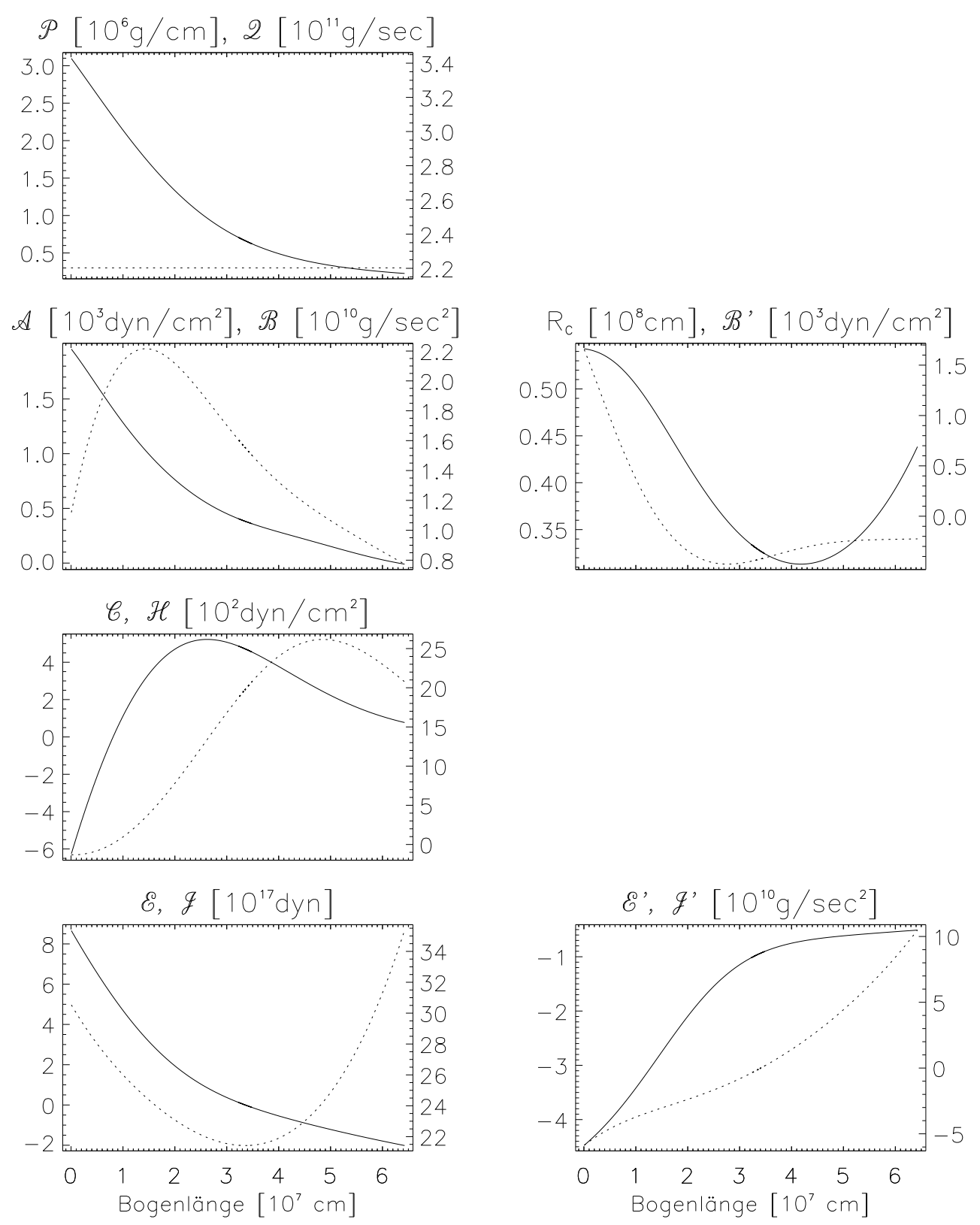

Abbildung 3.4: Koeffizienten zum Gleichungssystem (3.1) für das in Abbildung 2.4 dargestellte transkritische Gleichgewichtsmodell ohne Schock. Die Symmetrien der Koeffizienten zum Scheitelpunkt verschwinden für transkritische Gleichgewichte. Der Koeffizient $\mathcal{E}$ nimmt an einer Stelle, dem sogenannten kritischen Punkt, den Wert Null an. Dies führt zu einer prinzipiellen Schwierigkeit bei der Lösung der Stabilitätsgleichungen (3.1). 

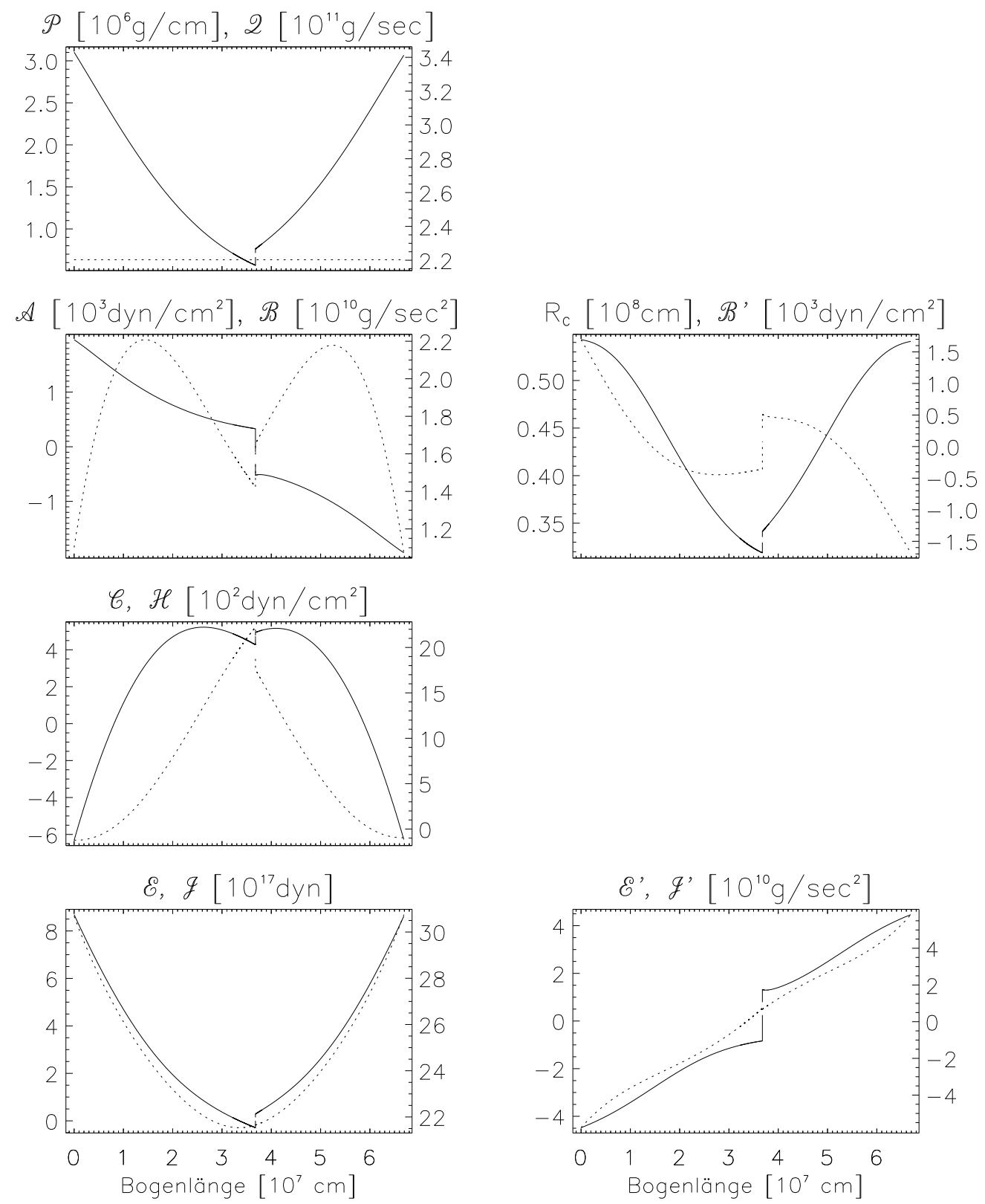

Abbildung 3.5: Koeffizienten im Störgleichungssystem (3.1) für das in Abbildung 2.5 dargestellte, transkritische Gleichgewichtsmodell mit Schock. Auch bei dieser Strömung nimmt der Koeffizient $\mathcal{E}$ den Wert Null an. Die Sprünge in den Koeffizienten treten am Ort des Schocks auf, sie sind Folge der Sprüngen der Gleichgewichtsgrößen am Schock. 

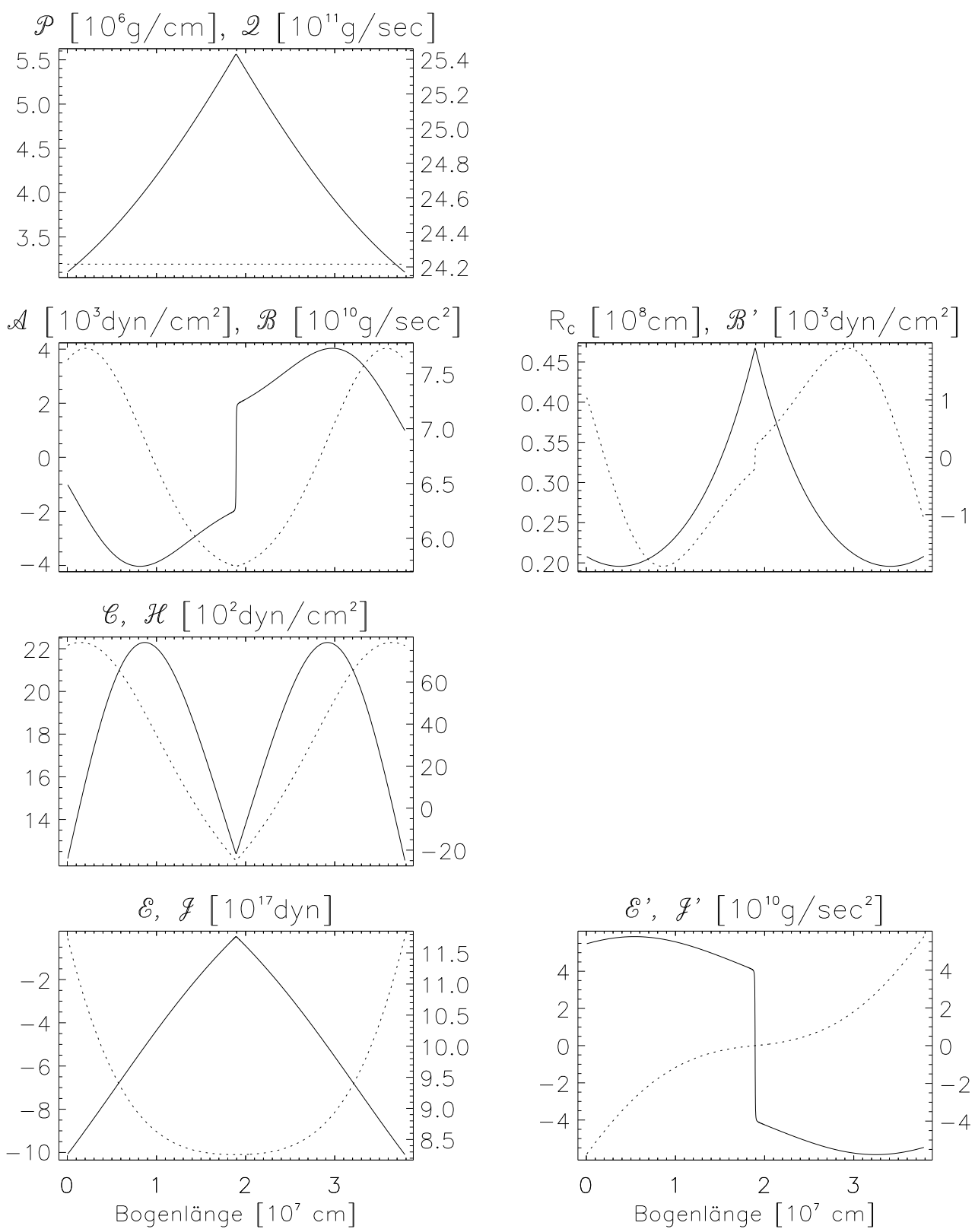

Abbildung 3.6: Koeffizienten zum Gleichungssystem (3.1) für das in Abbildung 2.6 dargestellte, überkritische Gleichgewichtsmodell mit einer Strömungsgeschwindigkeit im linken Röhrenfußpunkt von $781483 \mathrm{~cm} / \mathrm{sec}$. Der besondere Verlauf der Koeffizienten um den Röhrenscheitelpunkt hängt mit der sehr kleinen Differenz von Strömungs- und kritischer Geschwindigkeit dort zusammen. Alle Parameter in dieser Abbildung sind stetig differenzierbar. 

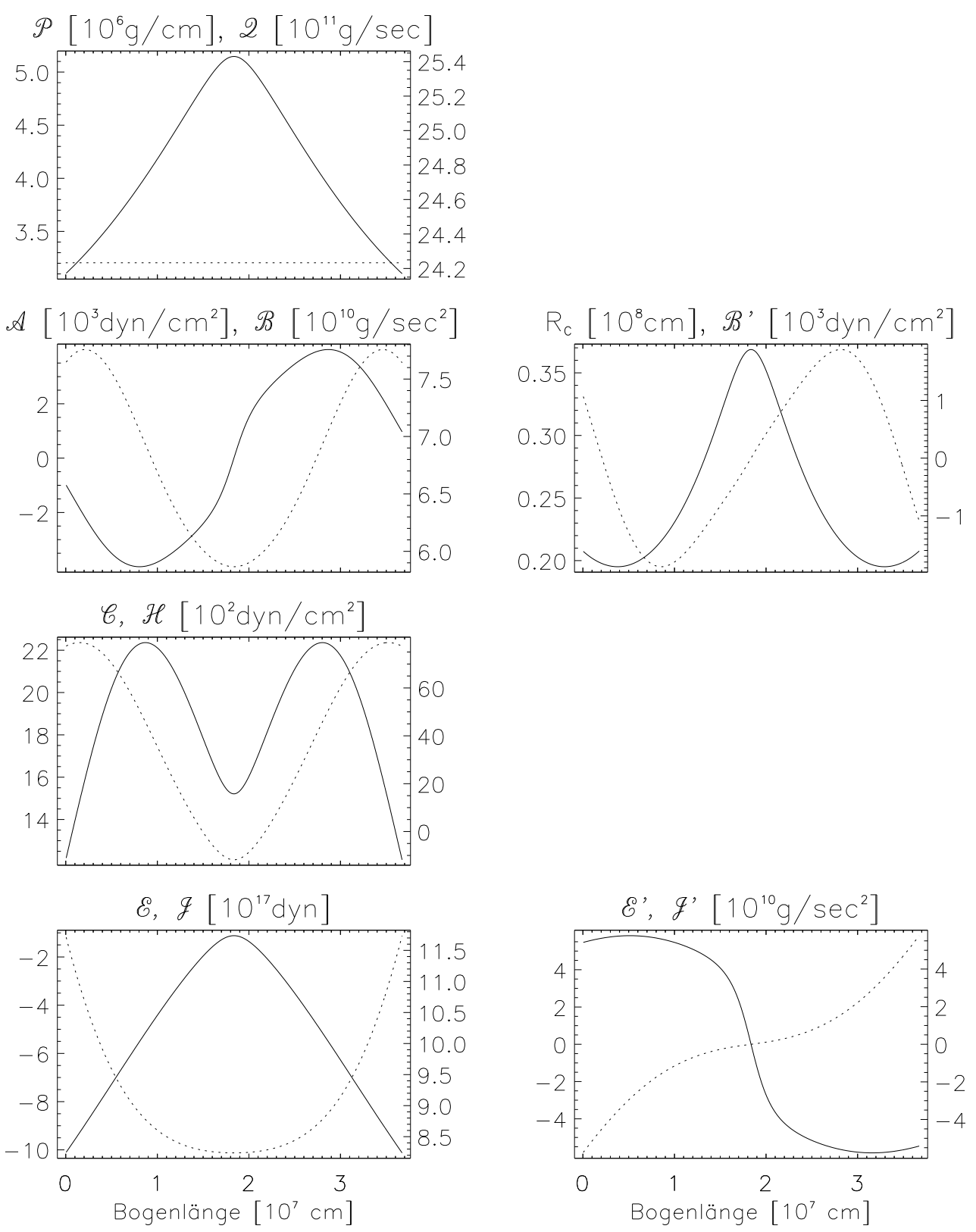

Abbildung 3.7: Koeffizienten zum Gleichungssystem (3.1) für das in Abbildung 2.7 dargestellte, überkritische Gleichgewichtsmodell mit einer Strömungsgeschwindigkeit im linken Röhrenfußpunkt von $782000 \mathrm{~cm} / \mathrm{sec}$. Bei dieser superkritischen Strömung ist die Differenz zwischen Strömungs- und kritischer Geschwindigkeit größer als bei dem zu den in Abbildung 3.6 gezeigten Koeffizienten gehörenden Modell. 


\subsubsection{Binormale Störungen}

Schmitt (1995; 1998) hat gezeigt, daß stationär durchströmte Flußröhren gegenüber binormalen Störungen stabil sind, wenn die Strömungsgeschwindigkeit überall kleiner als die Alfvéngeschwindigkeit ist. Dies ist bei den hier untersuchten Strömungen stets erfüllt. Somit wird die von den anderen beiden Gleichungen entkoppelte, dritte Gleichung im System (3.1) nicht weiter untersucht.

\subsubsection{Zeitabhängigkeit über Exponentialansatz}

Da alle in den Störungsgleichungen auftretenden Parameter zeitunabhängig sind, ist folgender Exponentialansatz zulässig

$$
\vec{\xi}=[\exp (i \omega t) \overrightarrow{\tilde{\xi}}(s)]_{r}
$$

$\omega$ und $\overrightarrow{\tilde{\xi}}(s)$ sind hierbei komplexwertige Größen, $i$ die imaginäre Einheit; der Index ,,r“ meint den Realteil. Im folgenden wird die Ortsabhängigkeit der Größe $\overrightarrow{\vec{\xi}}=\left(\begin{array}{c}\tilde{\eta} \\ \tilde{\epsilon}\end{array}\right)$ nicht angegeben. Das tangentiale und normale Störungen beschreibende Gleichungssystem wird

$$
\begin{array}{r}
-\mathcal{E} \frac{d^{2} \tilde{\eta}}{d s^{2}}+\left(2 i \omega \mathcal{Q}-\frac{d \mathcal{E}}{d s}\right) \frac{d \tilde{\eta}}{d s}-\left(\omega^{2} \mathcal{P}+\mathcal{C}\right) \tilde{\eta}-\mathcal{B} \frac{d \tilde{\epsilon}}{d s}-\left(2 i \omega \frac{\mathcal{Q}}{R_{C}}+\mathcal{A}\right) \tilde{\epsilon}=0 \\
-\mathcal{J} \frac{d^{2} \tilde{\epsilon}}{d s^{2}}+\left(2 i \omega \mathcal{Q}-\frac{d \mathcal{J}}{d s}\right) \frac{d \tilde{\epsilon}}{d s}-\left(\mu \omega^{2} \mathcal{P}+\mathcal{H}\right) \tilde{\epsilon}+\mathcal{B} \frac{d \tilde{\eta}}{d s}+\left(2 i \omega \frac{\mathcal{Q}}{R_{C}}-\mathcal{A}+\frac{d \mathcal{B}}{d s}\right) \tilde{\eta}=0
\end{array}
$$

oder alternativ in Operatorschreibweise

$$
\left(-\omega^{2} R+i \omega S-T\right)\left(\begin{array}{c}
\tilde{\eta} \\
\tilde{\epsilon}
\end{array}\right)=0
$$

mit

$$
\begin{aligned}
R & =\left(\begin{array}{cc}
\mathcal{P} & 0 \\
0 & \mu \mathcal{P}
\end{array}\right) \\
i S & =2 i \mathcal{Q}\left(\begin{array}{cc}
\frac{d}{d s} & -\frac{1}{R_{C}} \\
\frac{1}{R_{C}} & \frac{d}{d s}
\end{array}\right) \\
T & =\left(\begin{array}{cc}
\mathcal{E} \frac{d^{2}}{d s^{2}}+\frac{d \mathcal{E}}{d s} \frac{d}{d s}+\mathcal{C} & \mathcal{B} \frac{d}{d s}+\mathcal{A} \\
-\mathcal{B} \frac{d}{d s}+\mathcal{A}-\frac{d \mathcal{B}}{d s} & \mathcal{J} \frac{d^{2}}{d s^{2}}+\frac{d \mathcal{J}}{d s} \frac{d}{d s}+\mathcal{H}
\end{array}\right)
\end{aligned}
$$


Die Besonderheit der Stabilitätgleichung in dieser Form ist die von Schmitt (1995; 1998) gezeigte Hermitezität der Operatoren $R, i S$ und $T$. In den Hermitezitätsbeweis gehen die Randbedingungen für die Funktionen $\tilde{\eta}$ und $\tilde{\epsilon}$ ein. Im Fall einer von Null verschiedenen Strömungsgeschwindigkeit hat an beiden Rändern des Integrationsgebiets $\tilde{\eta}=0$ und $\tilde{\epsilon}=0 \mathrm{zu}$ gelten.

Als Folge der Hermitezität der Operatoren kommt man zu folgender Aussage (Schmitt, 1998; Frieman und Rotenberg, 1960): Ist $\omega=\iota$ Eigenwert, so sind auch $\omega=-\iota, \omega=\iota^{*}$ und $\omega=-\iota^{*}$ Eigenwerte. Der Exponent „,* meint hier und im folgenden das konjugiert Komplexe. Anhand des Ansatzes (3.3) erkennt man, daß für einen von Null verschiedenen Imaginarteil des Eigenwertes $\omega$ ein instabiler Schwingungsmode existiert.

Angenommen $\omega=\iota, \tilde{\eta}=\sigma$ und $\tilde{\epsilon}=\tau$ bilden eine Lösung der Stabilitätsgleichungen (3.4), so findet man durch Einsetzen von $\omega=-\iota^{*}, \tilde{\eta}=\sigma^{*}$ und $\tilde{\epsilon}=\tau^{*}$, daß diese ebenfalls eine Lösung der Gleichungen darstellen. Im Sonderfall von zum Röhrenscheitelpunkt $s_{\text {Scheitel }}$ symmetrischen Gleichgewichtsmodellen läßt sich weiterhin zeigen, daß zum Eigenwert $\omega=\iota^{*}$ die Funktionen $\tilde{\eta}=\sigma^{*}\left(2 s_{\text {Scheitel }}-s\right)$ und $\tilde{\epsilon}=-\tau^{*}\left(2 s_{\text {Scheitel }}-s\right)$ Lösungen der Stabilitätsgleichugen sind. Um dies zu zeigen, wird von den auf Seite 31 genannten Symmetrien der Koeffizienten der Stabilitätsgleichungen Gebrauch gemacht. Zu beachten sind die Gleichungen $d \tilde{\eta} / d s=-\sigma^{*^{\prime}}\left(2 s_{\text {Scheitel }}-s\right), d^{2} \tilde{\eta} / d s^{2}=\sigma^{*^{\prime \prime}}\left(2 s_{\text {Scheitel }}-s\right), d \tilde{\epsilon} / d s=\tau^{*^{\prime}}\left(2 s_{\text {Scheitel }}-s\right)$ und $d^{2} \tilde{\epsilon} / d s^{2}=-\tau^{*^{\prime \prime}}\left(2 s_{\text {Scheitel }}-s\right)$.

\subsection{Numerische Methoden}

Beim Gleichungssystem (3.4) handelt es sich um ein Eigenwertproblem von zwei komplexwertigen, gewöhnlichen Differentialgleichungen zweiter Ordnung. Zur numerischen Lösung solcher Probleme gibt es unterschiedliche Ansätze. Hier werden zwei Methoden verwendet, die zur Lösung von Randwertproblemen dienen:

- eine in Abschnitt 3.3.1 beschriebene Anfangswert-Methode (,Shooting“), deren Vorteil darin besteht, daß sie sich über eine variable Integrationsschrittweite dem Lösungsverhalten anpaßt und darum ein Maximum an Genauigkeit bietet. Diese Methode benötigt wenig Speicherplatz, da während der Integration die Lösung nicht an jedem Punkt bekannt sein muß.

- eine in Abschnitt 3.3.2 geschilderte globale Methode („Relaxation“), die bei hohem Speicherbedarf und im Normalfall kleinerer Genauigkeit die Möglichkeit bietet, sich besonderen numerischen Problemen wie z.B. numerischer Instabilität einzelner Gleichungen oder zusätzlichen Randbedingungen an inneren Punkten anzupassen.

Um die genannten Methoden anwenden zu können, wird das Eigenwertproblem in ein Randwertproblem überführt. Dies kann durch Hinzunahme der Differentialgleichung

$$
\frac{d \omega}{d s}=0
$$


für den Eigenwert $\omega$ erreicht werden (Press et al., 1992, S. 756).

Für die numerische Lösung wird das Gleichungssystem in zehn reellwertige Differentialgleichungen erster Ordnung umgeschrieben. Man erhält unter Einführung der neuen abhängigen Variablen $y_{1}, \ldots, y_{10}$ mit folgender Zuordnung

$$
\begin{array}{ll}
y_{1}=\tilde{\eta}_{r} ; \quad y_{2}=\tilde{\eta}_{i} ; \quad y_{3}=d \tilde{\eta}_{r} / d s ; & y_{4}=d \tilde{\eta}_{i} / d s \\
y_{5}=\tilde{\epsilon}_{r} ; \quad y_{6}=\tilde{\epsilon}_{i} ; \quad y_{7}=d \tilde{\epsilon}_{r} / d s ; & y_{8}=d \tilde{\epsilon}_{i} / d s \\
y_{9}=\omega_{r} ; & y_{10}=\omega_{i}
\end{array}
$$

das folgende Gleichungssystem

$$
\begin{aligned}
& \frac{d y_{1}}{d s}-y_{3}=0 \\
& \frac{d y_{2}}{d s}-y_{4}=0 \\
& \mathcal{E} \frac{d y_{3}}{d s}+\left(2 \mathcal{Q} y_{10}+\frac{d \mathcal{E}}{d s}\right) y_{3}+2 \mathcal{Q} y_{9} y_{4}+\left[\left(y_{9}^{2}-y_{10}^{2}\right) \mathcal{P}+\mathcal{C}\right] y_{1}- \\
& -2 y_{9} y_{10} \mathcal{P} y_{2}+\mathcal{B} y_{7}+\left(-\frac{2 \mathcal{Q}}{R_{C}} y_{10}+\mathcal{A}\right) y_{5}-\frac{2 \mathcal{Q}}{R_{C}} y_{9} y_{6}=0 \\
& \mathcal{E} \frac{d y_{4}}{d s}+\left(2 \mathcal{Q} y_{10}+\frac{d \mathcal{E}}{d s}\right) y_{4}-2 \mathcal{Q} y_{9} y_{3}+\left[\left(y_{9}^{2}-y_{10}^{2}\right) \mathcal{P}+\mathcal{C}\right] y_{2}+ \\
& +2 y_{9} y_{10} \mathcal{P} y_{1}+\mathcal{B} y_{8}+\left(-\frac{2 \mathcal{Q}}{R_{C}} y_{10}+\mathcal{A}\right) y_{6}+\frac{2 \mathcal{Q}}{R_{C}} y_{9} y_{5}=0 \\
& \frac{d y_{5}}{d s}-y_{7}=0 \\
& \frac{d y_{6}}{d s}-y_{8}=0 \\
& \frac{d y_{7}}{d s}+\frac{1}{\mathcal{J}}\left\{\left(2 \mathcal{Q} y_{10}+\frac{d \mathcal{J}}{d s}\right) y_{7}+2 \mathcal{Q} y_{9} y_{8}+\left[\mu\left(y_{9}^{2}-y_{10}^{2}\right) \mathcal{P}+\mathcal{H}\right] y_{5}-\right. \\
& \left.-2 \mu y_{9} y_{10} \mathcal{P} y_{6}-\mathcal{B} y_{3}+\left(\frac{2 \mathcal{Q}}{R_{C}} y_{10}+\mathcal{A}-\frac{d \mathcal{B}}{d s}\right) y_{1}+\frac{2 \mathcal{Q}}{R_{C}} y_{9} y_{2}\right\}=0 \\
& \frac{d y_{8}}{d s}+\frac{1}{\mathcal{J}}\left\{\left(2 \mathcal{Q} y_{10}+\frac{d \mathcal{J}}{d s}\right) y_{8}-2 \mathcal{Q} y_{9} y_{7}+\left[\mu\left(y_{9}^{2}-y_{10}^{2}\right) \mathcal{P}+\mathcal{H}\right] y_{6}+\right. \\
& \left.+2 \mu y_{9} y_{10} \mathcal{P} y_{5}-\mathcal{B} y_{4}+\left(\frac{2 \mathcal{Q}}{R_{C}} y_{10}+\mathcal{A}-\frac{d \mathcal{B}}{d s}\right) y_{2}-\frac{2 \mathcal{Q}}{R_{C}} y_{9} y_{1}\right\}=0 \\
& \frac{d y_{9}}{d s}=0 \\
& \frac{d y_{10}}{d s}=0
\end{aligned}
$$


Durch die angegebene Form der dritten und vierten Gleichung im System (3.6) wird an Punkten mit $\mathcal{E}=0$ eine Division durch Null und in deren Umgebung eine numerische Ungenauigkeit vermiedenf

\section{Randbedingungen}

Um die Hermitezität der Operatoren $R$, iS und $T$ aus Gleichung (3.5) und damit eine relativ einfache Deutbarkeit der Ergebnisse beibehalten zu können, wäre es wünschenswert, bei allen Rechnungen zur Stabilitätsanalyse den in Tabelle 3.1 genannten, auch bei Schmitt (1995; 1998) verwendeten Satz von Randwerten zu benutzen. Lösungen für transkritische

\begin{tabular}{|c|c|}
\hline linker Rand & rechter Rand \\
\hline \hline$\tilde{\eta}_{r}=0$ & $\tilde{\eta}_{r}=0$ \\
\hline$\tilde{\eta}_{i}=0$ & $\tilde{\eta}_{i}=0$ \\
\hline$\tilde{\epsilon}_{r}=0$ & $\tilde{\epsilon}_{r}=0$ \\
\hline$\tilde{\epsilon}_{i}=0$ & $\tilde{\epsilon}_{i}=0$ \\
\hline & $d \tilde{\epsilon}_{r} / d s=1$ \\
\hline & $d \tilde{\epsilon}_{i} / d s=1$ \\
\hline
\end{tabular}

Tabelle 3.1: Für die Stabilitätsanalyse unter-, über- sowie transkritischer Gleichgewichtsmodelle ohne Schock verwendete Randbedingungen. $d \tilde{\epsilon}_{r} / d s=1$ und $d \tilde{\epsilon}_{i} / d s=1$ werden aufgrund der Linearität des Gleichungssystems (3.4) in den abhängigen Variablen $\tilde{\eta}, \tilde{\eta}^{\prime}$, $\tilde{\epsilon}$ und $\tilde{\epsilon}^{\prime}$ gefordert und stellen nur eine Normierung dar. Die anderen Randbedingungen stehen für das sogenannte ,photosphärische line-tying“.

Gleichgewichtsmodelle mit Schock weisen jedoch bei diesen Bedingungen einen Sprung in den abhängigen Variablen $\tilde{\eta}_{r}$ und $\tilde{\eta}_{i}$ am kritischen Punkt auf, der im Gleichgewicht dem Röhrenscheitelpunkt entspricht. D.h. die abhängigen Variablen $d \tilde{\eta}_{r} / d s$ und $d \tilde{\eta}_{i} / d s$ nehmen dort den Wert „unendlich“ an. Um die Differenzierbarkeit der Funktionen $\tilde{\eta}_{r}, \tilde{\eta}_{i}, \tilde{\epsilon}_{r}, \tilde{\epsilon}_{i}, d \tilde{\eta}_{r} / d s$, $d \tilde{\eta}_{i} / d s, d \tilde{\epsilon}_{r} / d s$ und $d \tilde{\epsilon}_{i} / d s$ sicherzustellen, ist zu verhindern, daß die genannten Sprünge auftreten. Darüber hinaus dürfen keine Knicke in den abhängigen Variablen auftreten. Das gilt insbesondere am kritischen Punkt. Solche sogenannten Regularitätsbedingungen stellen formal Randbedingungen an inneren Punkten, in diesem Fall dem kritischen, dar (Press et al., 1992, S. 784ff). Um kein überbestimmtes Gleichungssystem zu bekommen, sind Randbedingungen aus Tabelle 3.1 aufzugeben. Als sinnvolle und auch gebräuchliche Randbedingungen werden $\tilde{\epsilon}_{r}=0$ und $\tilde{\epsilon}_{i}=0$ an beiden Fußpunkten beibehalten. Bei den unter Aufgabe von $\tilde{\eta}_{r}=0$ und $\tilde{\eta}_{i}=0$ entweder am linken oder am rechten Fußpunkt der Flußröhre erhaltenen Lösungen sind die abhängigen Variablen $d \tilde{\eta}_{r} / d s$ und $d \tilde{\eta}_{i} / d s$ am Ort des Schocks nicht

\footnotetext{
${ }^{4}$ Die hier angegebene Form des Systems (3.6) kann nur bei der Relaxation angewendet werden. Im Falle des Shooting sind die zweite und dritte Gleichung durch $\mathcal{E}$ zu dividieren.

${ }^{5} \mathrm{Schmitt}$ hat bei dem von ihm angewendeten numerischen Verfahrens eine andere Normierung verwendet als die durch die am rechten Rand gestellten Bedingungen $d \tilde{\epsilon}_{r} / d s=1$ und $d \tilde{\epsilon}_{i} / d s=1$ gegebene.
} 
differenzierbar, sie weisen Knicke auf. Dieses Verhalten ist durch die Diskontinuität der Koeffizienten dort bedingt, und die erhaltenen Lösungen stellen numerische Artefakte dar. Um es zu vermeiden, wird nötig, im Gleichgewichtsmodell den Schock nicht als einen Sprung an genau einem Punkt der Flußröhre zu behandeln, sondern ihn auf einen kleinen Bereich zu „,verschmieren“. Diese Vorgehensweise führt zu einem weiteren singulären Punkt des Differentialgleichungssystems, an dem wieder Regularität zu verlangen ist. Für transkritische Gleichgewichte mit Schock werden die in Tabelle 3.2 angegebenen Randbedingungen verwendet. Die verwendeten Randbedingungen bestimmen das Problem eindeutig.

\begin{tabular}{|c|c|c|c|}
\hline linker Rand & kritischer Punkt $(\mathcal{E}=0)$ & Schock $(\mathcal{E}=0)$ & rechter Rand \\
\hline \hline$\tilde{\epsilon}_{r}=0$ & & & $\tilde{\epsilon}_{r}=0$ \\
\hline$\tilde{\epsilon}_{i}=0$ & & & $\tilde{\epsilon}_{i}=0$ \\
\hline & $\mathcal{E} d^{2} \tilde{\eta}_{r} / d s^{2}=0$ & $\mathcal{E} d^{2} \tilde{\eta}_{r} / d s^{2}=0$ & \\
\hline & $\mathcal{E} d^{2} \tilde{\eta}_{i} / d s^{2}=0$ & $\mathcal{E} d^{2} \tilde{\eta}_{i} / d s^{2}=0$ & \\
\hline & & & $d \tilde{\epsilon}_{r} / d s=1$ \\
\hline & & & $d \tilde{\epsilon}_{i} / d s=1$ \\
\hline
\end{tabular}

Tabelle 3.2: Satz von Randbedingungen für transkritische Gleichgewichtsmodelle

\section{Entdimensionierung}

Aufgrund der begrenzten numerischen Rechengenauigkeit ist bei der Größenordnung der auftretenden Parameter und Variablen eine geeignete Entdimensionierung sinnvoll. Durch Verwendung der Skalen aus Tabelle 3.3 wird erreicht, daß Parameter und Variablen etwa die Größenordnung 1 annehmen.

\begin{tabular}{|l|r|}
\hline Skala & Wert \\
\hline \hline Längenskala & $3 \cdot 10^{7} \mathrm{~cm}$ \\
\hline Zeitskala & $50 \mathrm{sec}$ \\
\hline Massenskala & $10^{13} \mathrm{~g}$ \\
\hline
\end{tabular}

Tabelle 3.3: Skalen zur Entdimensionierung der im Gleichungssystem (3.6) auftretenden GröBen.

\subsubsection{Lösung des Eigenwertproblems mittels „Shooting“}

Bei der hier verwendeten Variante des „Shooting“ handelt es sich um ein z.B. in Press et al. (1992, S. 760ff) beschriebenes Verfahren: Ausgehend von zum Teil bekannten, zum Teil geratenen Randbedingungen wird von beiden Rändern des Integrationsgebietes bis an einen 
bestimmten inneren Punkt vorwärts integriert. An diesem Punkt müssen die erhaltenen Lösungen übereinstimmen. Faßt man die Differenz der Lösungen dort als Funktion der geratenen Randbedingungen auf, ist das Finden der wahren Werte für die unbekannten Randwerte zurückgeführt auf die Suche nach den Nullstellen dieser Funktion. Die Nullstellen lassen sich mit einem Sekanten- oder einem Newtonverfahren erhalten.

Als Vorwärtsintegrationsroutine hat sich die bereits bei der Behandlung des Gleichgewichts verwendete Runge-Kutta Routine nach Cash \& Karp (1990) bewährt. Für das Auffinden der Nullstellen wurde Broydens Methode (Broyden, 1965) verwendet.

Dieses Verfahren leistet im Fall von unter- und überkritischen Gleichgewichtslösungen gute Dienste. Es versagt aber bei transkritischen Modellen, selbst nach Ersetzen der genannten Runge-Kutta Routine durch ein für steife Differentialgleichungssysteme geeignetes Verfahren $($ Press et al., 1992, S. 738ff).

\subsubsection{Relaxation auf einem vorgegebenen Gitter}

Relaxation heißt die Methode, sich, ausgehend von einer möglichst guten Startlösung, durch iteratives Anbringen von Korrekturen der wahren Lösung anzunähern.

\section{Diskretisierung der Differentialquotienten}

Um ein Differentialgleichungssystem mittels einer Relaxation lösen zu können, sind die darin auftretenden Differentialquotienten in eine Form zu überführen, die numerisch handhabbar ist. Um die Verwendung von Gittern mit variabler Schrittweite zu erleichtern und eine spezielle Behandlung der Intervallgrenzen zu vermeiden, werden in dieser Arbeit zur Diskretisierung Zwei-Punkte Schemata (Ascher et al., 1995, S. 208ff) herangezogen. Im besonderen werden verwendet

- die Euler-vorwärts Diskretisierung $\frac{y_{i}^{(j+1)}-y_{i}^{(j)}}{h}=f_{i}\left(x^{(j)}, y_{i}^{(j)}\right)$

- die Euler-rückwärts Diskretisierung $\frac{y_{i}^{(j+1)}-y_{i}^{(j)}}{h}=f_{i}\left(x^{(j+1)}, y_{i}^{(j+1)}\right)$

- die Mittelpunkt Diskretisierung $\frac{y_{i}^{(j+1)}-y_{i}^{(j)}}{h}=f_{i}\left(x^{(j+1 / 2)}, \frac{1}{2}\left(y_{i}^{(j)}+y_{i}^{(j+1)}\right)\right)$

$x^{(j)}$ und $y_{i}^{(j)}$ bedeuten $x$ bzw. $y_{i}$ am $j$-ten Gitterpunkt.

Die erste und zweite sowie die fünfte bis achte Gleichung im System (3.6) sind in dieser Arbeit nach dem Mittelpunkt-Schema diskretisiert worden, da dieses Schema im Unterschied

${ }^{6}$ Da bei transkritischen Gleichgewichten der Koeffizient $\mathcal{E}$ in der Nähe der singulären Punkte dem Wert Null beliebig nahe kommt, werden dort einfache Vorwärtsintegrationen mit adaptiver Schrittweite numerisch instabil. Speziell für steife Differentialgleichungen entwickelte Methoden schaffen i.a. Abhilfe. Diese Methoden versagen hier aber, da sie am Punkt $\mathcal{E}=0$ die Division durch Null nicht leisten können. 
zu den anderen beiden eine Genauigkeit von zweiter Ordnung in der Schrittweite mit sich bringt. Für die neunte und zehnte Gleichung läßt sich nicht angeben, nach welchem Schema vorgegangen wurde, die verwendete Diskretisierung genügt jedem möglichen Zwei-Punkte Schema. Die dritte und vierte Gleichung wurden in Abhängigkeit vom Vorzeichen des Terms $\left(2 \mathcal{Q} y_{10}+d \mathcal{E} / d s\right) / \mathcal{E}$ diskretisiert; ist es positiv, findet die Rück-, sonst die Vorwärtsdiskretisierung nach Euler Verwendung. Diese Wahl der Diskretisierung wurde getroffen, weil für transkritische Strömungen im Bereich des kritischen Punktes der Betrag des Koeffizienten $\mathcal{E}$ vergleichbar dem Gitterabstand $h$ wird. Dann wäre aber die Diskretisierung nach dem Mittelpunktschema nicht mehr numerisch stabil, und man erhielte als Folge der Instabilität starke Oszillationen, die den gesamten Integrationsbereich des Gleichungssystems überdecken können. Die verwendete Diskretisierung ist numerisch stabil.

\section{Schrittweise Verbesserung der Lösung}

Angenommen es existiert eine Lösung $\tilde{y}_{i}^{(j)}$, die das diskretisierte Gleichungssystem schon recht gut erfüllt, aber den Genauigkeitsanforderungen noch nicht genügt. Dann gilt an den inneren Punkten $j=1, \ldots, M-1$

$$
\begin{aligned}
0 \approx & E_{i}^{(j)}=\tilde{y}_{i}^{(j+1)}-\tilde{y}_{i}^{(j)}- \\
& -\left.h f_{i}^{(j)}\left(y_{1}^{(j)}, \ldots, y_{N_{e}}^{(j)}, y_{1}^{(j+1)}, \ldots, y_{N_{e}}^{(j+1)}\right)\right|_{\tilde{y}_{1}^{(j)}, \ldots, \tilde{y}_{N_{e}}^{(j)}, \tilde{y}_{1}^{(j+1)}, \ldots, \tilde{y}_{N_{e}}^{(j+1)}}
\end{aligned}
$$

$E_{i}^{(j)}$ meint den Fehler der $i$-ten Gleichung am $j$-ten Gitterpunkt. Die unabhängige Variable taucht nicht auf, weil ihr Wert an jedem Punkt konstant ist. An den Rändern gilt entsprechend

$$
0 \approx E_{i}^{(0)}=\left.g_{i}\left(y_{1}^{(1)}, \ldots, y_{N_{e}}^{(1)}\right)\right|_{\tilde{y}_{1}^{(1)}, \ldots, \tilde{y}_{N_{e}}^{(1)}}
$$

und

$$
0 \approx E_{i}^{(M)}=\left.h_{i}\left(y_{1}^{(M)}, \ldots, y_{N_{e}}^{(M)}\right)\right|_{\tilde{y}_{1}^{(M)}, \ldots, \tilde{y}_{N_{e}}^{(M)}}
$$

Eine Taylorentwicklung der $E_{i}^{(j)}$ für die inneren Punkte ergibt

$$
\begin{aligned}
E_{i}^{(j) \approx} & \left.E_{i}^{(j)}\right|_{\tilde{y}_{1}^{(j)}, \ldots, \tilde{y}_{N_{e}}^{(j)}, \tilde{y}_{1}^{(j+1)}, \ldots, \tilde{y}_{N_{e}}^{(j+1)}}+ \\
& +\sum_{k=1}^{N_{e}}\left(\left.\Delta y_{k}^{(j)} \frac{\partial E_{i}^{(j)}}{\partial y_{k}^{(j)}}\right|_{\tilde{y}_{1}^{(j)}, \ldots, \tilde{y}_{N_{e}}^{(j)}, \tilde{y}_{1}^{(j+1)}, \ldots, \tilde{y}_{N_{e}}^{(j+1)}}+\right. \\
& \left.+\left.\Delta y_{k}^{(j+1)} \frac{\partial E_{i}^{(j)}}{\partial y_{k}^{(j+1)}}\right|_{\tilde{y}_{1}^{(j)}, \ldots, \tilde{y}_{N_{e}}^{(j)}, \tilde{y}_{1}^{(j+1)}, \ldots, \tilde{y}_{N_{e}}^{(j+1)}}\right)
\end{aligned}
$$


$\Delta y_{k}^{(j)}$ bedeutet die an die $\tilde{y}_{k}^{(j)}$ anzubringende Korrektur. Nun kann man an jedem inneren Gitterpunkt die $N_{e} \times 2 N_{e}$ Matrix

$$
S_{i, k}^{(j)}=\left\{\begin{array}{cc}
\left.\frac{\partial E_{i}^{(j)}}{\partial y_{k}^{(j)}}\right|_{\tilde{y}_{1}^{(j)}, \ldots, \tilde{y}_{N_{e}}^{(j)}, \tilde{y}_{1}^{(j+1)}, \ldots, \tilde{y}_{N_{e}}^{(j+1)}} & k=1, \ldots, N_{e} \\
\left.\frac{\partial E_{i}^{(j)}}{\partial y_{k-N_{e}}^{(j+1)}}\right|_{\tilde{y}_{1}^{(j)}, \ldots, \tilde{y}_{N_{e}}^{(j)}, \tilde{y}_{1}^{(j+1)}, \ldots, \tilde{y}_{N_{e}}^{(j+1)}} & k=N_{e}+1, \ldots, 2 N_{e}
\end{array}\right.
$$

definieren. Da die verbesserte Lösung eine wahre Lösung sein soll, erhält man

$$
-\left.E_{i}^{(j)}\right|_{\tilde{y}_{1}^{(j)}, \ldots, \tilde{y}_{N_{e}}^{(j)}, \tilde{y}_{1}^{(j+1)}, \ldots, \tilde{y}_{N_{e}}^{(j+1)}}=\sum_{k=1}^{N_{e}} S_{i, k}^{(j)} \Delta y_{k}^{(j)}+\sum_{k=N_{e}}^{2 N_{e}} S_{i, k}^{(j)} \Delta y_{k-N_{e}}^{(j+1)}
$$

An den Rändern ergibt sich analog

$$
\begin{aligned}
-\left.E_{i}^{(0)}\right|_{\tilde{y}_{1}^{(1)}, \ldots, \tilde{y}_{N_{e}}^{(1)}} & =\sum_{k=1}^{N_{e}} S_{i, k}^{(0)} \Delta y_{k}^{(1)} \\
S_{i, k}^{(0)} & =\left.\frac{\partial E_{i}^{(0)}}{\partial y_{k}^{(1)}}\right|_{\tilde{y}_{1}^{(1)}, \ldots, \tilde{y}_{N_{e}}^{(1)}}
\end{aligned}
$$

und

$$
\begin{aligned}
-\left.E_{i}^{(M)}\right|_{\tilde{y}_{1}^{(M)}, \ldots, \tilde{y}_{N_{e}}^{(M)}} & =\sum_{k=1}^{N_{e}} S_{i, k}^{(M)} \Delta y_{k}^{(M)} \\
S_{i, k}^{(M)} & =\left.\frac{\partial E_{i}^{(M)}}{\partial y_{k}^{(M)}}\right|_{\tilde{y}_{1}^{(M)}, \ldots, \tilde{y}_{N_{e}}^{(M)}}
\end{aligned}
$$

\section{Behandlung der singulären Punkte bei transkritischen Strömungen mit Schock}

Das diskretisierte Gleichungssystem umfaßt $M \cdot N_{e}$ Gleichungen. Davon sind $N_{e}$ durch Randbedingungen gegeben. Im Fall unter-, über- und transkritischer Strömungen ohne Schock sind das $N_{1}$ am linken und $N_{2}$ am rechten Rand mit $N_{1}+N_{2}=N_{e}$. Die Gleichungssystemmatrix hat die in Abbildung 3.8 gezeigte Form. Die durch im transkritischen Fall mit Schock auftretende Regularitätsforderungen an den singulären Punkten eingeführten inneren Randbedingungen ersetzen andere Randbedingungen. Behandelte man sie als zusätzliche Gleichungen, würde das Gleichungssystem überbestimmt, und man erhielte im allgemeinen keine Lösung. Das Gleichungssystem nimmt die in Abbildung 3.9 angegebene Form an. 


\begin{tabular}{|c|c|c|c|c|c|c|c|c|c|c|c|c|c|c|c||c|}
\hline $\mathrm{X}$ & $\mathrm{X}$ & $\mathrm{X}$ & $\mathrm{X}$ & & & & & & & & & & & & & $\mathrm{X}$ \\
\hline $\mathrm{X}$ & $\mathrm{X}$ & $\mathrm{X}$ & $\mathrm{X}$ & & & & & & & & & & & & & $\mathrm{X}$ \\
\hline $\mathrm{X}$ & $\mathrm{X}$ & $\mathrm{X}$ & $\mathrm{X}$ & $\mathrm{X}$ & $\mathrm{X}$ & $\mathrm{X}$ & $\mathrm{X}$ & & & & & & & & & $\mathrm{X}$ \\
\hline $\mathrm{X}$ & $\mathrm{X}$ & $\mathrm{X}$ & $\mathrm{X}$ & $\mathrm{X}$ & $\mathrm{X}$ & $\mathrm{X}$ & $\mathrm{X}$ & & & & & & & & & $\mathrm{X}$ \\
\hline $\mathrm{X}$ & $\mathrm{X}$ & $\mathrm{X}$ & $\mathrm{X}$ & $\mathrm{X}$ & $\mathrm{X}$ & $\mathrm{X}$ & $\mathrm{X}$ & & & & & & & & & $\mathrm{X}$ \\
\hline $\mathrm{X}$ & $\mathrm{X}$ & $\mathrm{X}$ & $\mathrm{X}$ & $\mathrm{X}$ & $\mathrm{X}$ & $\mathrm{X}$ & $\mathrm{X}$ & & & & & & & & & $\mathrm{X}$ \\
\hline & & & & $\mathrm{X}$ & $\mathrm{X}$ & $\mathrm{X}$ & $\mathrm{X}$ & $\mathrm{X}$ & $\mathrm{X}$ & $\mathrm{X}$ & $\mathrm{X}$ & & & & & $\mathrm{X}$ \\
\hline & & & & $\mathrm{X}$ & $\mathrm{X}$ & $\mathrm{X}$ & $\mathrm{X}$ & $\mathrm{X}$ & $\mathrm{X}$ & $\mathrm{X}$ & $\mathrm{X}$ & & & & & $\mathrm{X}$ \\
\hline & & & & $\mathrm{X}$ & $\mathrm{X}$ & $\mathrm{X}$ & $\mathrm{X}$ & $\mathrm{X}$ & $\mathrm{X}$ & $\mathrm{X}$ & $\mathrm{X}$ & & & & & $\mathrm{X}$ \\
\hline & & & & $\mathrm{X}$ & $\mathrm{X}$ & $\mathrm{X}$ & $\mathrm{X}$ & $\mathrm{X}$ & $\mathrm{X}$ & $\mathrm{X}$ & $\mathrm{X}$ & & & & & $\mathrm{X}$ \\
\hline & & & & & & & & $\mathrm{X}$ & $\mathrm{X}$ & $\mathrm{X}$ & $\mathrm{X}$ & $\mathrm{X}$ & $\mathrm{X}$ & $\mathrm{X}$ & $\mathrm{X}$ & $\mathrm{X}$ \\
\hline & & & & & & & & $\mathrm{X}$ & $\mathrm{X}$ & $\mathrm{X}$ & $\mathrm{X}$ & $\mathrm{X}$ & $\mathrm{X}$ & $\mathrm{X}$ & $\mathrm{X}$ & $\mathrm{X}$ \\
\hline & & & & & & & & $\mathrm{X}$ & $\mathrm{X}$ & $\mathrm{X}$ & $\mathrm{X}$ & $\mathrm{X}$ & $\mathrm{X}$ & $\mathrm{X}$ & $\mathrm{X}$ & $\mathrm{X}$ \\
\hline & & & & & & & & & & & & $\mathrm{X}$ & $\mathrm{X}$ & $\mathrm{X}$ & $\mathrm{X}$ & $\mathrm{X}$ \\
\hline & & & & & & & & & & & & $\mathrm{X}$ & $\mathrm{X}$ & $\mathrm{X}$ & $\mathrm{X}$ & $\mathrm{X}$ \\
\hline
\end{tabular}

Abbildung 3.8: Erweiterte Gleichungssystemmatrix im Fall $M=4, N_{e}=4, N_{1}=2$ und $N_{2}=2$. Es sind keine Randbedingungen an inneren Punkten gestellt. Die von Null verschiedenen Matrixelemente sind durch ein „,X“ gekennzeichnet.

\subsubsection{Test der Numerik}

Die verwendeten numerischen Methoden sind auf zwei Arten getestet worden:

- Die Ergebnisse für unter- und überkritische Strömungen stimmen für Schieß- und Relaxationsverfahren überein.

- Die erhaltenen Ergebnisse der Stabilitätsanalyse wurden wieder in das bestimmende Gleichungssystem (3.4) eingesetzt, wobei einmal numerisch zu differenzieren war. Diese Gleichungen sind mit guter Genauigkeit erfüllt.

Ein Konsistenztest ergibt sich noch aus dem in Abschnitt 3.4 vorgestellen globalen Stabilitätskriterium. Mittels der Gleichung (3.8) wird dort aus den Eigenfunktionen der Eigenwert $\omega$ berechnet. In allen in dieser Arbeit vorgestellten Rechnungen ist Übereinstimmung von dem so berechneten Eigenwert mit dem aus der Lösung des Differentialgleichungssystems gegeben.

\subsection{Stabilitätskriterium aus den integrierten Stabilitätsgleichungen}

In der klassischen Mechanik läßt sich der Energiesatz durch ein Integral der Bewegungsgleichung gewinnen. Dazu wird die Bewegungsgleichung skalar mit dem Geschwindigkeitsvektor 


\begin{tabular}{|c|c|c|c|c|c|c|c|c|c|c|c|c|c|c|c||c|}
\hline $\mathrm{X}$ & $\mathrm{X}$ & $\mathrm{X}$ & $\mathrm{X}$ & & & & & & & & & & & & & $\mathrm{X}$ \\
\hline $\mathrm{X}$ & $\mathrm{X}$ & $\mathrm{X}$ & $\mathrm{X}$ & $\mathrm{X}$ & $\mathrm{X}$ & $\mathrm{X}$ & $\mathrm{X}$ & & & & & & & & & $\mathrm{X}$ \\
\hline $\mathrm{X}$ & $\mathrm{X}$ & $\mathrm{X}$ & $\mathrm{X}$ & $\mathrm{X}$ & $\mathrm{X}$ & $\mathrm{X}$ & $\mathrm{X}$ & & & & & & & & & $\mathrm{X}$ \\
\hline $\mathrm{X}$ & $\mathrm{X}$ & $\mathrm{X}$ & $\mathrm{X}$ & $\mathrm{X}$ & $\mathrm{X}$ & $\mathrm{X}$ & $\mathrm{X}$ & & & & & & & & & $\mathrm{X}$ \\
\hline $\mathrm{X}$ & $\mathrm{X}$ & $\mathrm{X}$ & $\mathrm{X}$ & $\mathrm{X}$ & $\mathrm{X}$ & $\mathrm{X}$ & $\mathrm{X}$ & & & & & & & & & $\mathrm{X}$ \\
\hline & & & & $\mathrm{X}$ & $\mathrm{X}$ & $\mathrm{X}$ & $\mathrm{X}$ & $\mathrm{X}$ & $\mathrm{X}$ & $\mathrm{X}$ & $\mathrm{X}$ & & & & & $\mathrm{X}$ \\
\hline & & & & $\mathrm{X}$ & $\mathrm{X}$ & $\mathrm{X}$ & $\mathrm{X}$ & & & & & & & & & $\mathrm{X}$ \\
\hline & & & & $\mathrm{X}$ & $\mathrm{X}$ & $\mathrm{X}$ & $\mathrm{X}$ & $\mathrm{X}$ & $\mathrm{X}$ & $\mathrm{X}$ & $\mathrm{X}$ & & & & & $\mathrm{X}$ \\
\hline & & & & $\mathrm{X}$ & $\mathrm{X}$ & $\mathrm{X}$ & $\mathrm{X}$ & $\mathrm{X}$ & $\mathrm{X}$ & $\mathrm{X}$ & $\mathrm{X}$ & & & & & $\mathrm{X}$ \\
\hline & & & & $\mathrm{X}$ & $\mathrm{X}$ & $\mathrm{X}$ & $\mathrm{X}$ & $\mathrm{X}$ & $\mathrm{X}$ & $\mathrm{X}$ & $\mathrm{X}$ & & & & & $\mathrm{X}$ \\
\hline & & & & & & & & $\mathrm{X}$ & $\mathrm{X}$ & $\mathrm{X}$ & $\mathrm{X}$ & $\mathrm{X}$ & $\mathrm{X}$ & $\mathrm{X}$ & $\mathrm{X}$ & $\mathrm{X}$ \\
\hline & & & & & & & & $\mathrm{X}$ & $\mathrm{X}$ & $\mathrm{X}$ & $\mathrm{X}$ & & & & & $\mathrm{X}$ \\
\hline & & & & & & & & $\mathrm{X}$ & $\mathrm{X}$ & $\mathrm{X}$ & $\mathrm{X}$ & $\mathrm{X}$ & $\mathrm{X}$ & $\mathrm{X}$ & $\mathrm{X}$ & $\mathrm{X}$ \\
\hline & & & & & & & & $\mathrm{X}$ & $\mathrm{X}$ & $\mathrm{X}$ & $\mathrm{X}$ & $\mathrm{X}$ & $\mathrm{X}$ & $\mathrm{X}$ & $\mathrm{X}$ & $\mathrm{X}$ \\
\hline & & & & & & & & $\mathrm{X}$ & $\mathrm{X}$ & $\mathrm{X}$ & $\mathrm{X}$ & $\mathrm{X}$ & $\mathrm{X}$ & $\mathrm{X}$ & $\mathrm{X}$ & $\mathrm{X}$ \\
\hline & & & & & & & & & & & & $\mathrm{X}$ & $\mathrm{X}$ & $\mathrm{X}$ & $\mathrm{X}$ & $\mathrm{X}$ \\
\hline
\end{tabular}

Abbildung 3.9: Erweiterte Gleichungssystemmatrix im Fall $M=4, N_{e}=4, N_{1}=1$ und $N_{2}=1$. Je eine Randbedingung rechts und links wurden durch zwei innere ersetzt. Da die inneren Randbedingungen für Forderungen stehen, bei denen benachbarte Punkte keine Rolle spielen, sind sie im Schema daran zu erkennen, daß nur $N_{e}$ Elemente pro Zeile von Null verschieden sind. 
multipliziert und über die Zeit integriert. Im Rahmen der MHD sind Bernstein et al. (1958) bei linearen Stabilitätsuntersuchungen statischer Gleichgewichte und Frieman und Rotenberg (1960) bei ebensolchen stationärer Gleichgewichte ähnlich verfahren. Als Resultat wurden Kriterien für die Stabilität der untersuchten Gleichgewichte erhalten.

Skalare Multiplikation der Bewegungsgleichung (3.4) mit dem konjugiert Komplexen des Vektors $\overrightarrow{\tilde{\xi}}$ und Integration über die Bogenlänge führt auf die Gleichung

$$
-a \omega^{2}+2 b \omega-c=0
$$

mit den Abkürzungen

$$
\begin{aligned}
a= & \int_{0}^{s_{1}} \mathcal{P}\left(\tilde{\eta} \tilde{\eta}^{*}+\mu \tilde{\epsilon} \tilde{\epsilon}^{*}\right) d s \\
b= & i \mathcal{Q} \int_{0}^{s_{1}}\left(\frac{d \tilde{\eta}}{d s} \tilde{\eta}^{*}+\frac{d \tilde{\epsilon}}{d s} \tilde{\epsilon}^{*}+\frac{\tilde{\eta} \tilde{\epsilon}^{*}-\tilde{\epsilon} \tilde{\eta}^{*}}{R_{C}}\right) d s \\
c= & \int_{0}^{s_{1}}\left(\mathcal{E} \frac{d^{2} \tilde{\eta}}{d s^{2}} \tilde{\eta}^{*}+\frac{d \mathcal{E}}{d s} \frac{d \tilde{\eta}}{d s} \tilde{\eta}^{*}\right) d s+\int_{0}^{s_{1}}\left(\mathcal{J} \frac{d^{2} \tilde{\epsilon}}{d s^{2}} \tilde{\epsilon}^{*}+\frac{d \mathcal{J}}{d s} \frac{d \tilde{\epsilon}}{d s} \tilde{\epsilon}^{*}\right) d s+ \\
& -\int_{0}^{s_{1}}\left[\mathcal{B}\left(\frac{d \tilde{\eta}}{d s} \tilde{\epsilon}^{*}-\frac{d \tilde{\epsilon}}{d s} \tilde{\eta}^{*}\right)+\frac{d \mathcal{B}}{d s} \tilde{\eta} \tilde{\epsilon}^{*}\right] d s+ \\
& +\int_{0}^{s_{1}} \mathcal{C} \tilde{\eta} \tilde{\eta}^{*} d s+\int_{0}^{s_{1}} \mathcal{H} \tilde{\epsilon} \tilde{\epsilon}^{*} d s+\int_{0}^{s_{1}} \mathcal{A}\left(\tilde{\epsilon} \tilde{\eta}^{*}+\tilde{\eta} \tilde{\epsilon}^{*}\right) d s
\end{aligned}
$$

$a$ ist reellwertig und immer positiv. $b$ und $c$ sind im allgemeinen komplexwertig. Gleichung (3.7) hat die Lösungen

$$
\omega=\frac{1}{a}\left(b \pm \sqrt{b^{2}-a c}\right)
$$

\subsubsection{Allgemeines Stabilitätskriterium}

Definiert man

$$
d=b^{2}-a c
$$

so gilt nach der Eulerschen Relation

$$
d=d_{R} \exp \left(i d_{\phi}\right)
$$

wobei $d_{R}$ und $d_{\phi}$ reelle Zahlen sind. $d_{R}$ ist als Absolutbetrag von $d$ positiv oder Null. Man erhält anstelle der Gleichungen (3.8) als Lösung

$$
\omega=\frac{1}{a}\left[b \pm \exp \left(i \frac{d_{\phi}}{2}\right) \sqrt{d_{R}}\right]
$$


Instabilität liegt aufgrund des Ansatzes (3.3) vor, wenn der Imaginärteil von $\omega$ kleiner als Null ist. Wegen der Gleichungen (3.9) gilt

$$
\omega_{i}=\frac{1}{a}\left[b_{i} \pm \sin \left(\frac{d_{\phi}}{2}\right) \sqrt{d_{R}}\right]
$$

Stabilitätskriterien bekommt man aus einer Fallunterscheidung

- Der Imaginärteil von $b$ ist negativ. $\Longrightarrow$ Das untersuchte Gleichgewicht ist instabil.

- $b$ ist Element der reellen Zahlen.

- $\sin \left(\frac{d \phi}{2}\right) \sqrt{d_{R}} \neq 0 . \Longrightarrow$ Das untersuchte Gleichgewicht ist instabil.

- $\frac{d_{\phi}}{2}=0 . \Longrightarrow$ Das untersuchte Gleichgewicht ist stabil in bezug auf den betrachteten Mode.

- Der Imaginärteil von $b$ ist positiv

- $\sqrt{d_{R}} \leq b_{i} . \Longrightarrow$ Das untersuchte Gleichgewicht ist stabil in bezug auf den betrachteten Mode.

- $\left|\sin \left(\frac{d_{\phi}}{2}\right)\right| \sqrt{d_{R}}>b_{i} . \Longrightarrow$ Das untersuchte Gleichgewicht ist instabil.

- $\frac{d_{\phi}}{2}=0 . \Longrightarrow$ Das untersuchte Gleichgewicht ist stabil in bezug auf den betrachteten Mode.

\subsubsection{Ein Sonderfall}

Für die Strömungen, bei denen als Randbedingungen $\tilde{\eta}=\tilde{\epsilon}=0$ an beiden Rändern verwendet wurden, läßt sich ein einfacheres Stabilitätskriterium ableiten. Vorab zwei nützliche Gleichungen in den zwei komplexen Zahlen $\phi$ und $\psi$

$$
\begin{aligned}
\phi \psi^{*}+\phi^{*} \psi & =2\left(\phi_{r} \psi_{r}+\phi_{i} \psi_{i}\right) \\
\phi \psi^{*}-\phi^{*} \psi & =2 i\left(\phi_{i} \psi_{r}-\phi_{r} \psi_{i}\right)
\end{aligned}
$$

Behauptung. Mit $\tilde{\eta}(0)=\tilde{\eta}\left(s_{1}\right)=\tilde{\epsilon}(0)=\tilde{\epsilon}\left(s_{1}\right)$ ist b Element der reellen Zahlen

Beweis. Durch partielle Integration gewinnt man

$$
\int_{0}^{s_{1}} \frac{d \tilde{\eta}}{d s} \tilde{\eta}^{*} d s=\left[\tilde{\eta} \tilde{\eta}^{*}\right]_{0}^{s_{1}}-\int_{0}^{s_{1}} \tilde{\eta} \frac{d \tilde{\eta}^{*}}{d s} d s
$$

Wegen der zweiten Gleichung in (3.10) gilt für $\tilde{\eta}=0$ an den Rändern des Integrationsgebiets

$$
2 \int_{0}^{s_{1}} \frac{d \tilde{\eta}}{d s} \tilde{\eta}^{*} d s=\int_{0}^{s_{1}}\left(\frac{d \tilde{\eta}}{d s} \tilde{\eta}^{*}-\tilde{\eta} \frac{d \tilde{\eta}^{*}}{d s}\right) d s=2 i \int_{0}^{s_{1}}\left(\frac{d \tilde{\eta}_{i}}{d s} \tilde{\eta}_{r}-\frac{d \tilde{\eta}_{r}}{d s} \tilde{\eta}_{i}\right) d s
$$


Das Integral

$$
\int_{0}^{s_{1}} \frac{d \tilde{\epsilon}}{d s} \tilde{\epsilon}^{*} d s
$$

wird analog behandelt. Aufgrund der zweiten Gleichung (3.10) gilt

$$
\tilde{\eta} \tilde{\epsilon}^{*}-\tilde{\epsilon} \tilde{\eta}^{*}=2 i\left(\tilde{\eta}_{i} \tilde{\epsilon}_{r}-\tilde{\eta}_{r} \tilde{\epsilon}_{i}\right)
$$

Behauptung. Mit $\tilde{\eta}(0)=\tilde{\eta}\left(s_{1}\right)=\tilde{\epsilon}(0)=\tilde{\epsilon}\left(s_{1}\right)$ ist c Element der reellen Zahlen

Beweis. Partielle Integration liefert

$$
\int_{0}^{s_{1}}\left(\mathcal{E} \frac{d^{2} \tilde{\eta}}{d s^{2}} \tilde{\eta}^{*}+\frac{d \mathcal{E}}{d s} \frac{d \tilde{\eta}}{d s} \tilde{\eta}^{*}\right) d s=\left[\mathcal{E} \frac{d \tilde{\eta}}{d s} \tilde{\eta}^{*}\right]_{0}^{s_{1}}-\int_{0}^{s_{1}} \mathcal{E} \frac{d \tilde{\eta}}{d s} \frac{d \tilde{\eta}^{*}}{d s} d s
$$

Analog folgt

$$
\int_{0}^{s_{1}}\left(\mathcal{J} \frac{d^{2} \tilde{\epsilon}}{d s^{2}} \tilde{\epsilon}^{*}+\frac{d \mathcal{J}}{d s} \frac{d \tilde{\epsilon}}{d s} \tilde{\epsilon}^{*}\right) d s=\left[\mathcal{J} \frac{d \tilde{\epsilon}}{d s} \tilde{\epsilon}^{*}\right]_{0}^{s_{1}}-\int_{0}^{s_{1}} \mathcal{J} \frac{d \tilde{\epsilon}}{d s} \frac{d \tilde{\epsilon}^{*}}{d s} d s
$$

Durch partielle Integration erhält man

$$
-\int_{0}^{s_{1}}\left[\mathcal{B}\left(\frac{d \tilde{\eta}}{d s} \tilde{\epsilon}^{*}-\frac{d \tilde{\epsilon}}{d s} \tilde{\eta}^{*}\right)+\frac{d \mathcal{B}}{d s} \tilde{\eta} \tilde{\epsilon}^{*}\right] d s=-\left[\mathcal{B} \tilde{\eta} \tilde{\epsilon}^{*}\right]_{0}^{s_{1}}+\int_{0}^{s_{1}} \mathcal{B}\left(\tilde{\eta} \frac{d \tilde{\epsilon}^{*}}{d s}+\tilde{\eta}^{*} \frac{d \tilde{\epsilon}}{d s}\right) d s
$$

Hierbei ist das letzte Integral wegen der ersten Gleichung (3.10) Element der reellen Zahlen. Die Integrale

$$
\int_{0}^{s_{1}} \mathcal{C} \tilde{\eta} \tilde{\eta}^{*} d s
$$

und

$$
\int_{0}^{s_{1}} \mathcal{H} \tilde{\epsilon} \tilde{\epsilon}^{*} d s
$$

sind Element der reellen Zahlen. Wieder aufgrund der ersten Gleichung (3.10) ist das Integral

$$
\int_{0}^{s_{1}} \mathcal{A}\left(\tilde{\epsilon} \tilde{\eta}^{*}+\tilde{\eta} \tilde{\epsilon}^{*}\right) d s
$$

Element der reellen Zahlen.

Man erhält als Stabilitätskriterium

$$
\frac{b^{2}}{a}>c
$$

Dies Kriterium vereinfacht sich wegen der zweiten Gleichung (3.2) für statische Gleichgewichte $\mathrm{zu}$

$$
0>c
$$

Beide Kritierien findet man bei Frieman und Rotenberg (1960) und Schmitt (1995; 1998). 


\subsection{Ergebnisse}

Aufgrund des iterativen Charakters der beiden verwendeten numerischen Methoden zur Lösung der Stabilitätsgleichungen kann die Vollständigkeit der in den folgenden Abschnitten angegebenen Sätze von Eigenwerten und Eigenfunktionen nicht garantiert werden.

\subsubsection{Subkritische Strömungen}

Die untersuchten Gleichgewichtsmodelle mit unterkritischen Strömungen zeigen für wachsende Strömungsgeschwindigkeit am linken Fußpunkt der Röhre einen dem Betrag nach größer werdenden Imaginärteil des Eigenwertes $\omega$. Das läßt eine Instabilität infolge steigender Strömungsgeschwindigkeit vermuten. Eine Betrachtung der Ergebnisse über die Gleichung (3.11) macht aber deutlich, daß in allen untersuchten Fällen Stabilität herrscht. Der scheinbare Widerspruch zwischen einem dem Betrag nach ansteigenden Imaginärteil von $\omega$ und der gefundenen Stabilität läßt sich über den Unterschied in der Größenordnung zwischen Real- und Imaginärteil von $\omega$ aufklären. Es handelt sich dabei um eine relative numerische Ungenauigkeit im Bereich von $\approx 10^{-6}$. Tabelle 3.4 gibt Eigenwerte und die zugehörigen

\begin{tabular}{|c|c|c|c|}
\hline$v_{0}[\mathrm{~cm} / \mathrm{sec}]$ & $\omega[1 / \mathrm{sec}]$ & $\left(b^{2}-a c\right) / a^{2}\left[10^{-4} / \mathrm{sec}^{2}\right]$ & siehe Abb. \\
\hline \hline 1 & $\left(0.0158,-3.65 \cdot 10^{-14}\right)$ & 2.50 & 3.10 \\
\hline & $\left(0.0334,1.76 \cdot 10^{-14}\right)$ & 11.1 & \\
\hline & $\left(0.0567,1.12 \cdot 10^{-13}\right)$ & 32.2 & 3.11 \\
\hline & $\left(0.0622,-4.25 \cdot 10^{-15}\right)$ & 38.6 & 3.12 \\
\hline 40000 & $\left(0.0143,-4.58 \cdot 10^{-9}\right)$ & 2.87 & 3.13 \\
\hline & $\left(0.0327,2.18 \cdot 10^{-9}\right)$ & 10.2 & \\
\hline & $\left(0.0526,-4.61 \cdot 10^{-9}\right)$ & 32.7 & 3.14 \\
\hline & $\left(0.0638,-2.03 \cdot 10^{-10}\right)$ & 40.0 & \\
\hline 71000 & $\left(0.00754,1.40 \cdot 10^{-8}\right)$ & 6.29 & 3.15 \\
\hline & $\left(0.0238,-2.71 \cdot 10^{-8}\right)$ & 11.7 & \\
\hline & $\left(0.0362,2.34 \cdot 10^{-8}\right)$ & 24.1 & \\
\hline
\end{tabular}

Tabelle 3.4: Eigenwerte aus Stabilitätsrechnungen zu den in Abbildungen 2.1-2.3 dargestellten unterkritischen Gleichgewichten.

Werte des Terms $\left(b^{2}-a c\right) / a^{2}$ aus dem globalen Stabilitätskriterium (3.11) zu den in den Abbildungen 2.1-2.3 gezeigten Gleichgewichtsmodellen an. Die angegebenen Eigenwerte sind zu jeweils einem betrachteten Gleichgewicht nach ansteigendem Realteil von $\omega$ sortiert. Die ebenfalls erhaltenen Eigenwerte mit negativem Realteil sind nicht aufgeführt. Bei den hier beschriebenen Stabilitätsanalysen unterkritischer Modelle wurden Eigenwerte im Bereich $10^{-5} / \mathrm{sec}<\omega_{r}<0.05 / \mathrm{sec}$ und $10^{-4} / \mathrm{sec}<\left|\omega_{i}\right|<0.02 / \mathrm{sec}$ als Startwerte vorgegeben. Die Schrittweite, mit der die genannten Intervalle durchlaufen wurden, betrug für $\omega_{r}$ wie für $\omega_{i}$ 
$10^{-3} /$ sec. Die zu einigen dieser Eigenwerte gehörigen Störungen $\eta$ und $\epsilon$ werden in den Abbildungen 3.10-3.15 dargestellt. Mit groberer Schrittweite ist der Bereich, in dem der Realteil von $\omega$ variiert wurde bis zu Werten von $\omega_{r}<0.5$ nach Eigenwerten durchsucht worden. Es wurden weitere stabile Oberschwingungen gefunden, die hier nicht mit angegeben sind.

Bei Gleichgewichtsmodellen, die mit nennenswerter Geschwindigkeit durchströmt werden, findet man im Gegensatz zu denen, bei denen die Strömungsgeschwindigkeit überall vernachlässigbar klein gegenüber der kritischen Geschwindigkeit ist, daß die Gleichgewichtslösung zu keinem Zeitpunkt gilt. Es liegt immer an einem Ort der Flußröhre eine Störung vor. Die Abbildungen $3.12-3.15$ erwecken dabei den Eindruck, daß sich die Ortsstörungen tangential und normal zur Röhre mit von der Bogenlänge abhängiger Amplitude entlang der Flußröhre bewegen. Es handelt sich dabei nicht um ebene Wellen, sondern um Wellenpakete, deren Ausbreitungsgeschwindigkeit vom Ort abhängt. Bei allen untersuchten unterkritischen Gleichgewichtsmodellen breiten sich die tangentialen und normalen Ortsstörungen zum Eigenwert mit dem kleinsten positiven $\omega_{r}$ entgegen der Strömung aus. Die Geschwindigkeit, mit der die Knotenlinie sich bei diesem Moden bewegt, fällt mit zunehmender Annäherung an den Scheitelpunkt, in dem man die größten Werte der Gleichgewichtsströmungsgeschwindigkeit findet, ab. Bei den Oberschwingungen werden die Verhältnisse schnell komplizierter. So erkennt man anhand von Abbildung 3.13, daß sich die normale Ortsstörung in Teilen der Röhre mit der Strömung ausbreitet. Eine einfache Zuordnung dieser wellenartigen Bewegung zu bekannten MHD-Wellen, dem langsamen oder dem schnellen magnetoakustischen Mode, ist nicht möglich. Je weiter sich im Röhrenscheitelpunkt die Strömungsgeschwindigkeit $v$ der kritischen Geschwindigkeit $c_{T}$ nähert, desto steiler verläuft $\eta$ in der Umgebung des Scheitelpunkts.

\subsubsection{Transkritische Strömung ohne Schock}

Der kritische Punkt des untersuchten Gleichgewichtsmodells liegt bei $s \approx 3.354 \cdot 10^{7} \mathrm{~cm}$. Zu diesem in Abbildung 2.4 dargestellten Gleichgewichtsmodell wurden mehrere Stabilitätsanalysen gerechnet, die sich darin unterscheiden, bis zu welcher Bogenlänge $s_{1}$ die Integration erfolgte. An diesem Endpunkt waren die Randbedingungen $\tilde{\eta}=0$ und $\tilde{\epsilon}=0$ zu erfüllen. Im allgemeinen treten am kritischen Punkt die gleichen Probleme auf, wie in Abschnitt 12 für transkritische Strömungen mit Schock geschildert. Im Unterschied zu diesen wurde jedoch ein regulärer Mode mit verschwindendem Realteil des Eigenwertes $\omega$ gefunden. Aus der auf Seite 40 gemachten Aussage „Ist $\omega=\iota$ Eigenwert, dann ist auch $\omega=\iota^{*}$ Eigenwert“ folgt unabhängig vom gefundenen Vorzeichen des Imaginärteils von $\omega$ die Existenz eines nicht schwingenden monoton anwachsenden Modes. Die Instabilität des transkritischen Gleichgewichts ohne Schock konnte damit ohne das Stellen einer Regularitätsforderung gezeigt werden. In Tabelle 3.5 findet sich eine Aufstellung der gefundenen Moden in Abhängigkeit des Endpunktes der Integration. Außer dem Eigenwert $\omega$ ist der Ausdruck $\left(b^{2}-a c\right) / a^{2}$ angegeben. Anhand dieses Ausdrucks kann man nach Gleichung (3.11) ablesen, daß alle angegebenen Moden nach dem globalen Kriterium instabil sind. Dieses Kriterium liefert als Lösung der Gleichung (3.8) im allgemeinen zwei Eigenwerte. Neben den in der Tabelle genannten Werten $\omega$, die durch 

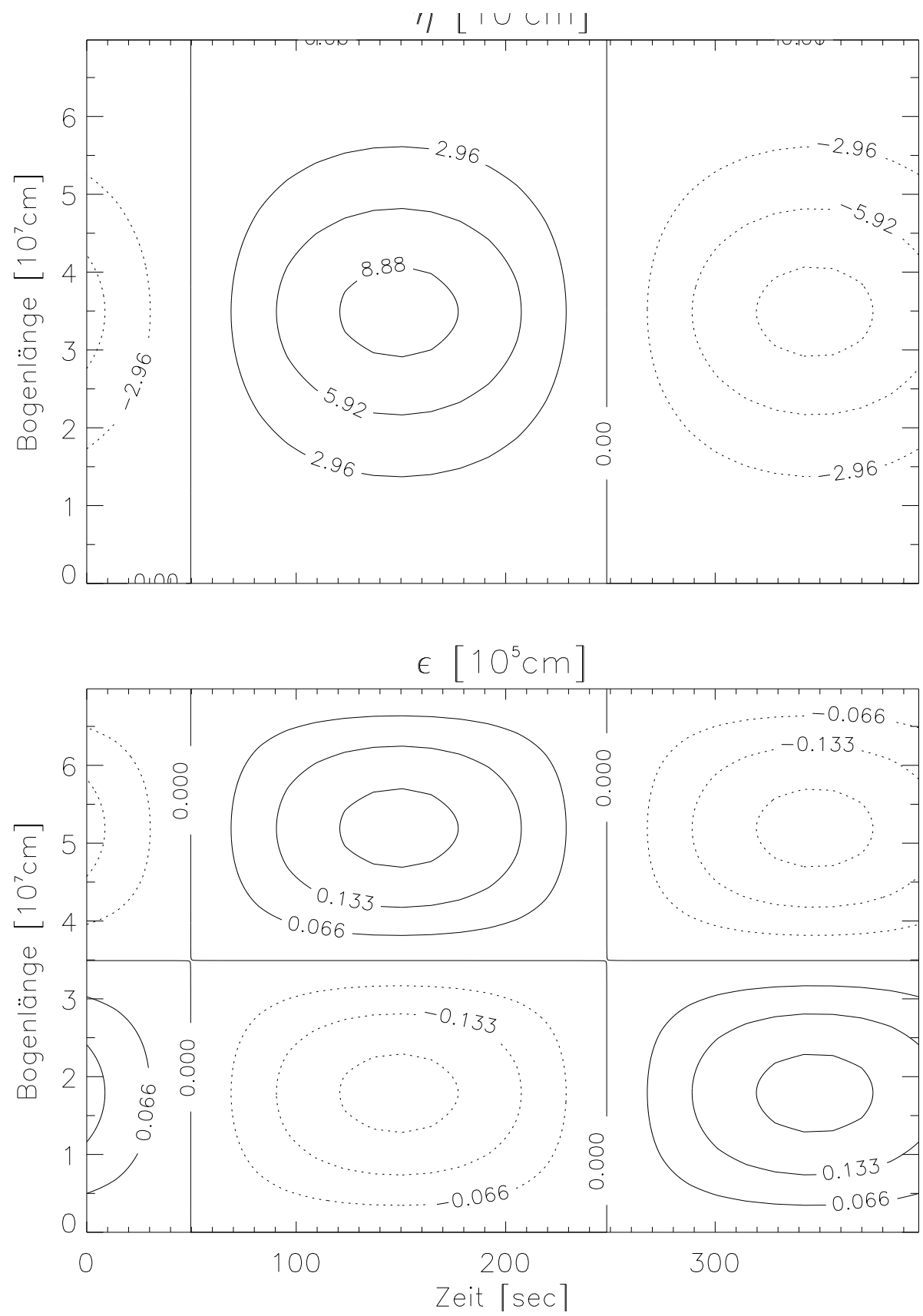

Abbildung 3.10: Konturlinien der Störungen im Ort der Flußröhre tangential (oben) und normal zur Flußröhre zum Eigenwert $\omega=\left(0.0158,-3.65 \cdot 10^{-14}\right) / \mathrm{sec}$. Die Störungen gehören zum in Abbildung 2.1 gezeigten Gleichgewicht mit $v_{0}=1 \mathrm{~cm} / \mathrm{sec}$. Die absolute Amplitude der Ortsstörungen ist, wie in den folgenden Darstellungen, aufgrund der Linearität des Gleichungssystems (3.4) ohne Bedeutung. Die kurzen Striche an jeder Konturlinie zeigen, wie auch bei den folgenden Konturliniengraphiken, in Richtung kleinerer Werte. Die Flußröhre oszilliert um das Gleichgewicht. 

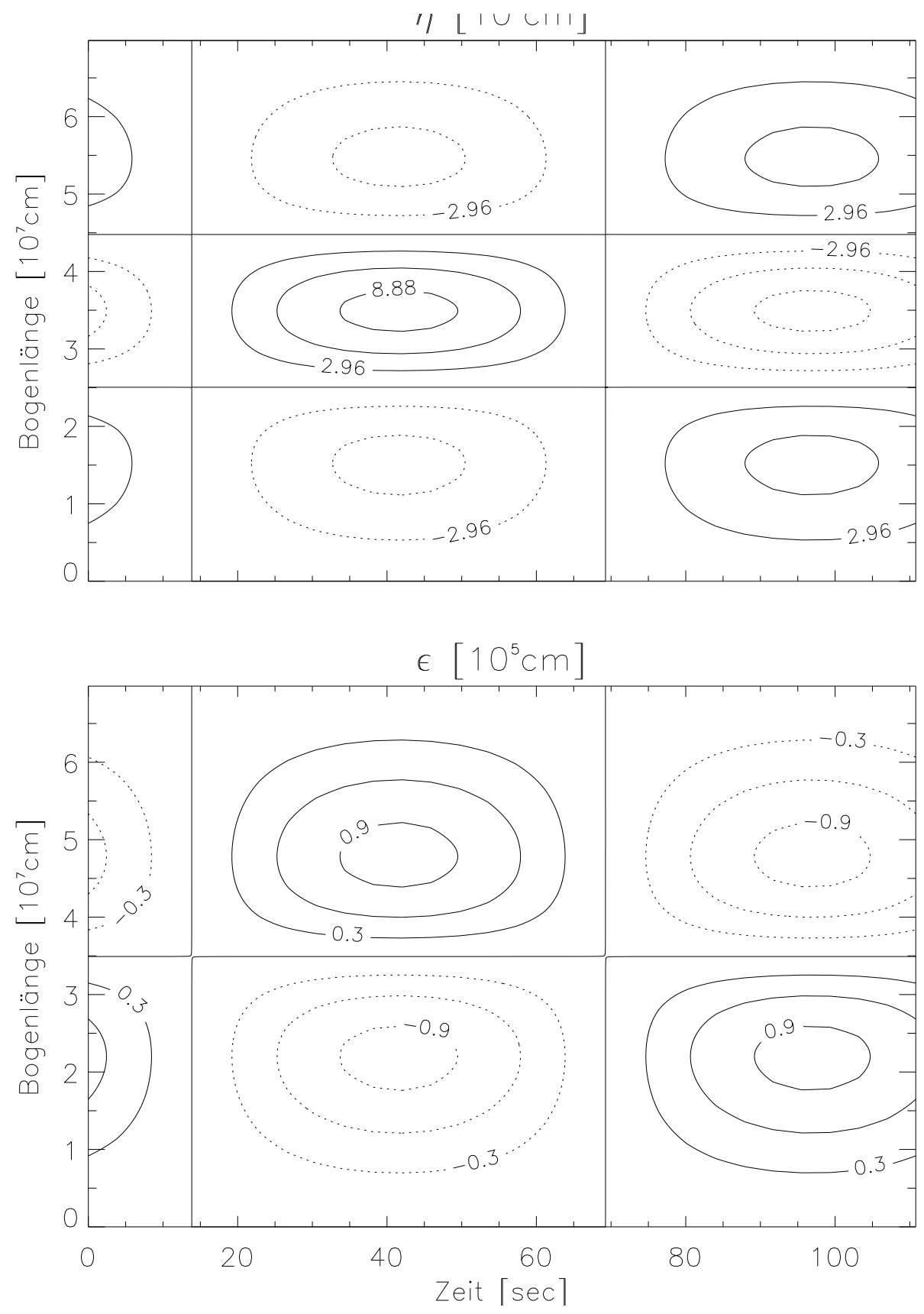

Abbildung 3.11: Konturlinien der Störungen im Ort tangential (oben) und normal zur Flußröhre zum Eigenwert $\omega=\left(0.0567,1.12 \cdot 10^{-13}\right) / \mathrm{sec}$. Die Störungen gehören zum in Abbildung 2.1 gezeigten Gleichgewicht mit $v_{0}=1 \mathrm{~cm} / \mathrm{sec}$. Es handelt sich hier einen höheren Mode als in Abbildung 3.10. Auch hier oszilliert die Röhre um ihr Gleichgewicht. 

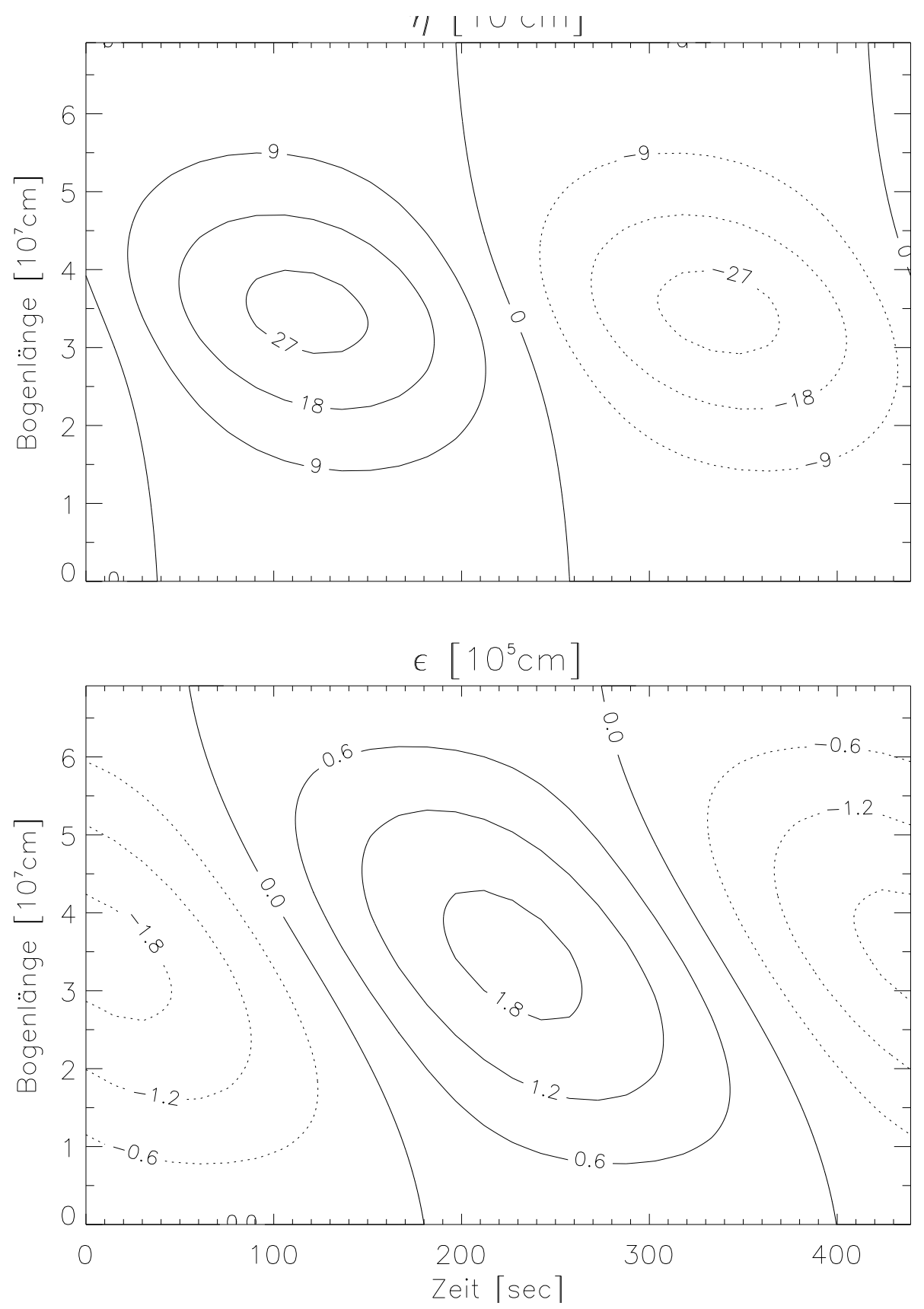

Abbildung 3.12: Konturlinien der Störungen im Ort tangential (oben) und normal zur Flußröhre zum Eigenwert $\omega=\left(0.0143,-4.58 \cdot 10^{-9}\right) / \mathrm{sec}$. Die Störungen gehören zum in Abbildung 2.2 gezeigten Gleichgewicht mit $v_{0}=40000 \mathrm{~cm} / \mathrm{sec}$. Wie auch bei allen anderen Störungsrechnungen zu mit nennenswerter Strömungsgeschwindigkeit durchströmten Gleichgewichtsmodellen findet keine Oszillation um das Gleichgewicht statt. Die Linien verschwindender Ortsstörungen laufen der Strömung entgegen, wobei eine Phasenverschiebung zwischen tangentialer und normaler Störung auftritt. 

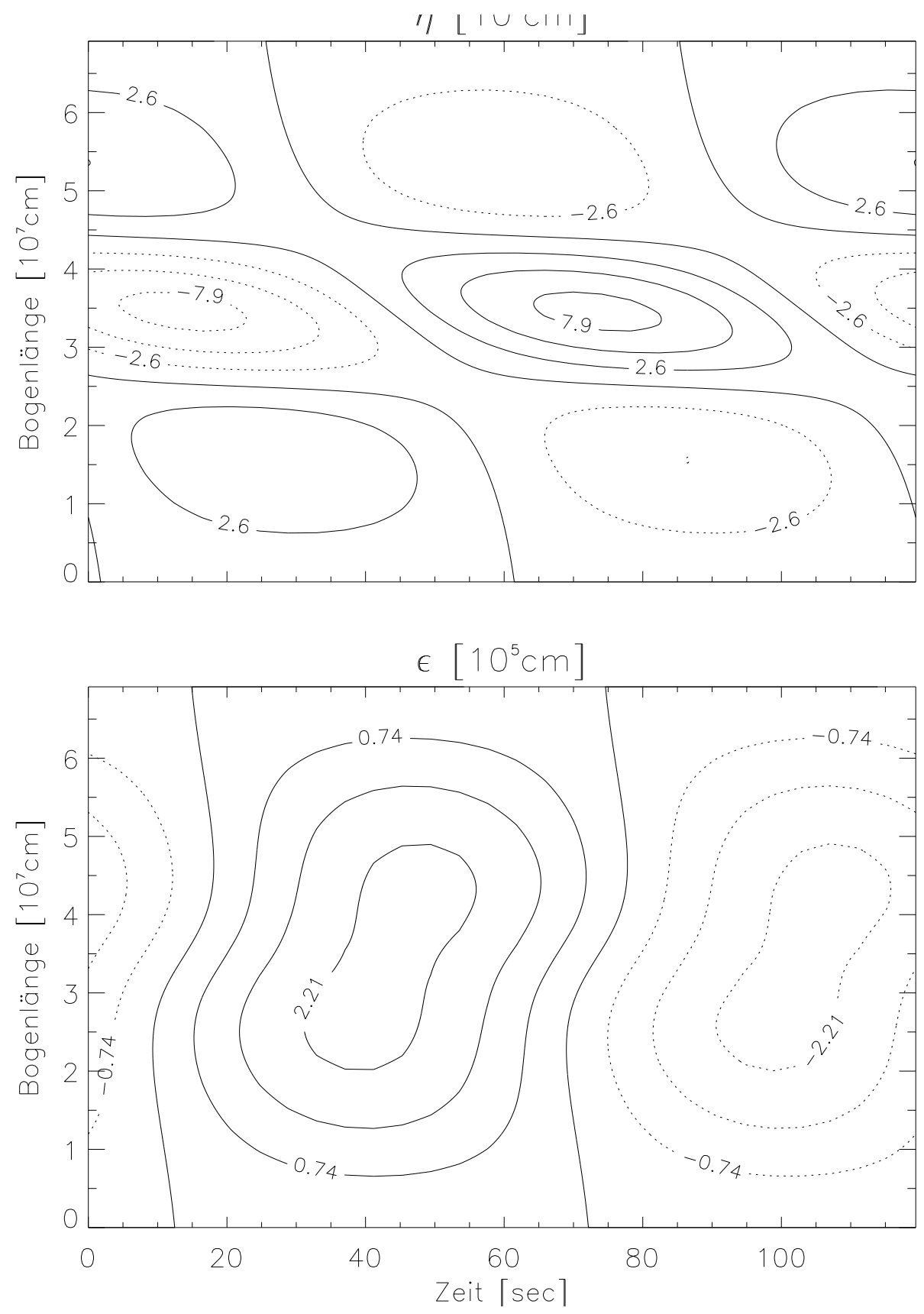

Abbildung 3.13: Konturlinien der Störungen im Ort tangential (oben) und normal zur Flußröhre zum Eigenwert $\omega=\left(0.0526,-4.61 \cdot 10^{-9}\right) /$ sec. Die Störungen gehören zum in Abbildung 2.2 gezeigten Gleichgewicht mit $v_{0}=40000 \mathrm{~cm} / \mathrm{sec}$. Es handelt sich um eine Oberschwingung zum Mode aus Abbildung 3.12. Auffällig ist, daß bei diesem Mode die Knotenlinie der normale Ortsstörung zum Teil mit zum Teil entgegen der Strömungsrichtung verläuft. 

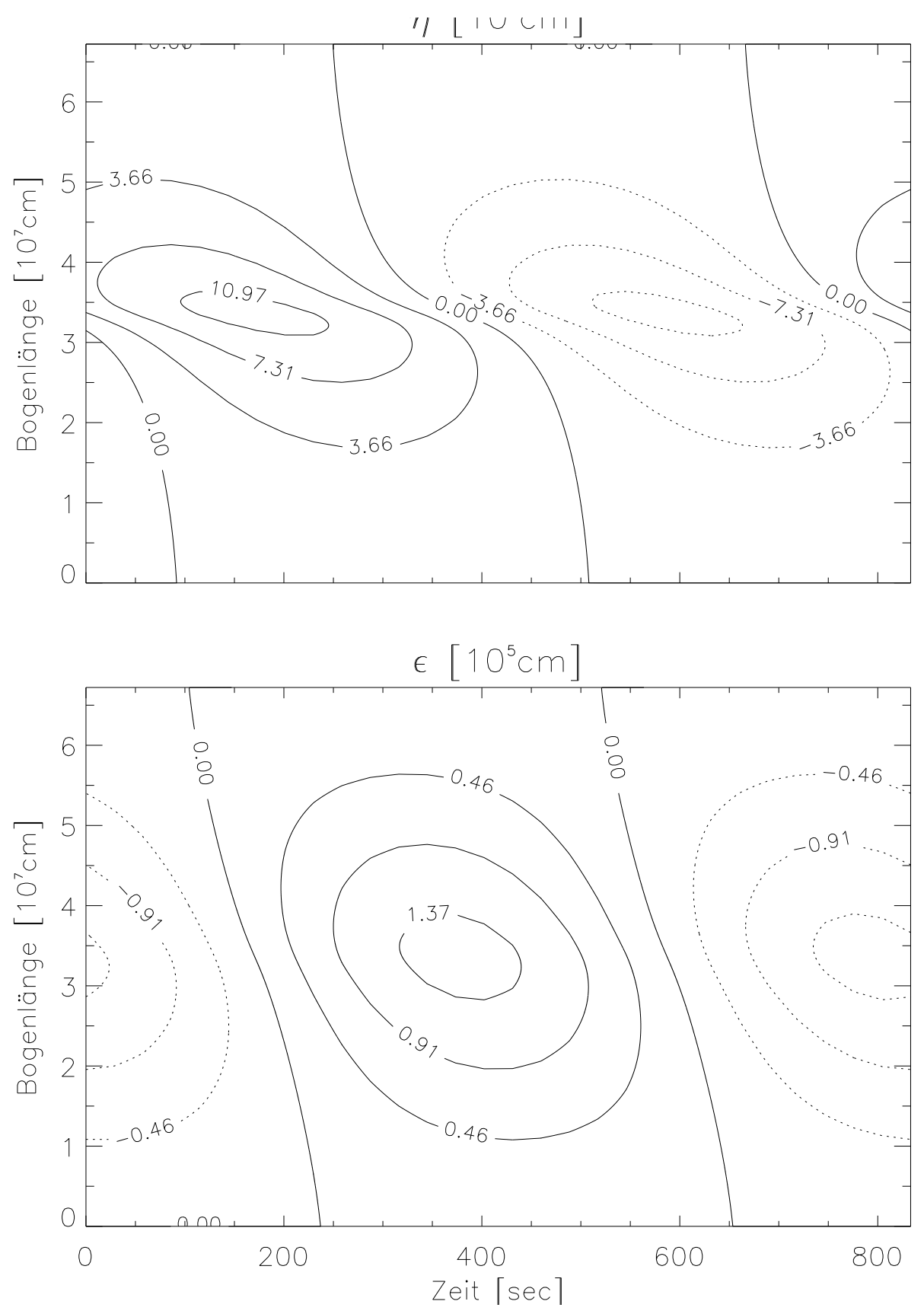

Abbildung 3.14: Konturlinien der Störungen im Ort tangential (oben) und normal zur Flußröhre zum Eigenwert $\omega=\left(0.00754,1.40 \cdot 10^{-8}\right) /$ sec. Die Störungen gehören zum in Abbildung 2.3 gezeigten Gleichgewicht mit $v_{0}=71000 \mathrm{~cm} / \mathrm{sec}$. In der Umgebung des Röhrenscheitelpunkts verläuft $\eta$ steiler als in unterkritischen Modellen mit kleinerem $v_{0}$. Die Knotenlinie der Ortsstörung bewegt sich entgegen der Strömung. 

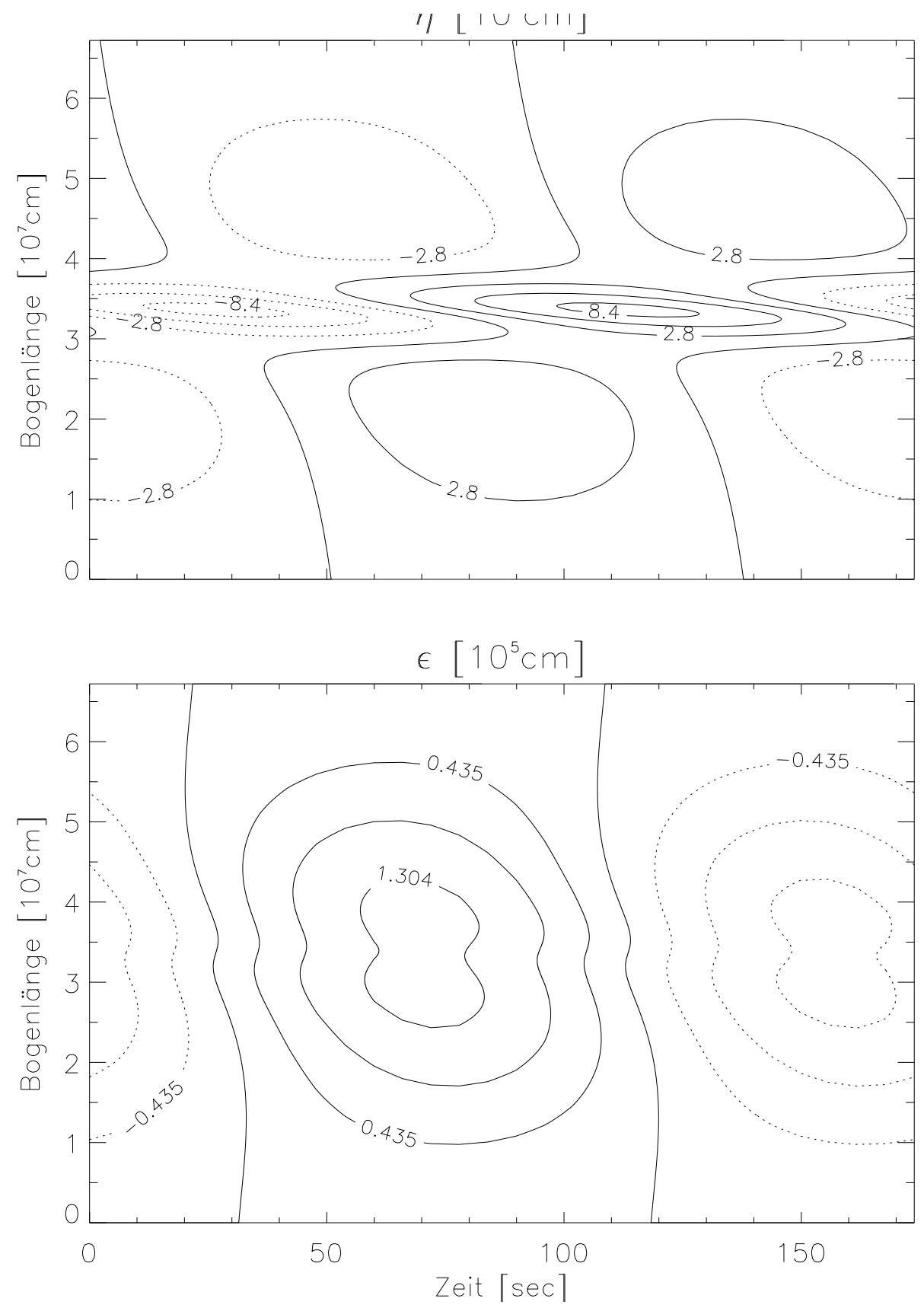

Abbildung 3.15: Konturlinien der Störung im Ort tangential (oben) und normal zur Flußröhre zum Eigenwert $\omega=\left(0.0362,2.34 \cdot 10^{-8}\right) /$ sec. Die Störungen gehören zum in Abbildung 2.3 gezeigten Gleichgewicht mit $v_{0}=71000 \mathrm{~cm} / \mathrm{sec}$. Auch hier fällt der steile Verlauf von $\eta$ in der Umgebung des Scheitelpunkts auf. 


\begin{tabular}{|c|c|c|c|}
\hline$s_{1}\left[10^{7} \mathrm{~cm}\right]$ & $\omega[1 / \mathrm{sec}]$ & $\left(b^{2}-a c\right) / a^{2}\left[10^{-4} / \mathrm{sec}^{2}\right]$ & siehe Abb. \\
\hline \hline 3.5 & $\left(-3.11 \cdot 10^{-12}, 0.317\right)$ & $-1.01 \cdot 10^{3}$ & \\
\hline 4.0 & $\left(3.32 \cdot 10^{-14}, 0.0606\right)$ & -36.7 & \\
\hline 4.5 & $\left(-6.68 \cdot 10^{-12}, 0.0854\right)$ & -72.9 & 3.16 \\
\hline 5.0 & $\left(-4.17 \cdot 10^{-14}, 0.0617\right)$ & -38.0 & \\
\hline 5.5 & $\left(1.16 \cdot 10^{-12}, 0.0454\right)$ & -20.6 & \\
\hline 6.0 & $\left(8.84 \cdot 10^{-14}, 0.0413\right)$ & -17.1 & 3.17 \\
\hline 6.4262 & $\left(-3.37 \cdot 10^{-13}, 0.0432\right)$ & -18.7 & \\
\hline
\end{tabular}

Tabelle 3.5: Eigenwerte $\omega$ aus Stabilitätsanalysen der in Abbildung 2.4 gezeigten transkritischen Strömung ohne Schock in Abhängigkeit von der Bogenlänge $s_{1}$, bis zu der die Integration erfolgte.

eine Relaxation erhalten wurden, ist, wie erwartet'], jedesmal auch $\omega^{*}$ Eigenwert. Zwei der in Tabelle 3.5 gegebenen Moden sind in den Abbildungen 3.16 und 3.17 graphisch dargestellt. Bei allen gefundenen Moden liegt das Maximum der Auslenkung im überkritischen Teilgebiet. Die Anwachs- bzw. Zerfallsraten liegen im Bereich von $10^{-1}$ bis $10^{-2} / \mathrm{sec}$. Innerhalb von 10 bis $100 \mathrm{sec}$ sind also die Auslenkungen auf das $e$-fache angewachsen bzw. das $1 / e$-fache abgefallen.

Es wurden auch Stabilitätsanalysen zu dem eigentlich transkritischen Gleichgewichtsmodell ohne Schock gerechnet, die vor dem kritischen Punkt abbrechen, also nicht mehr transkritisch sind. Alle bei diesen Rechnungen gefundenen Moden sind stabil. Einige Eigenwerte sind in Tabelle 3.6 angegeben. Mit zunehmender Länge der Flußröhre nimmt der Realteil der gefundenen Eigenwerte ab. Die Abbildungen 3.18-3.21 zeigen eine Auswahl der gefundenen Moden zu unterschiedlichen Werten von $s_{1}$. Man findet Eigenfunktionen, die denen unterkritischer Strömungen ähnlich sind. Wie bei diesen findet sich für die Moden mit den kleinsten positiven Werten $\omega_{r}$ ein Verlauf der Ortsstörungen entgegen der Strömung. Bei den beiden in den Abbildungen 3.20 und 3.21 dargestellten Moden fällt der steile Verlauf von $\eta$ in der Nähe des Röhrenscheitelpunkts auf, in dessen Umgebung sich $v$ der kritischen Geschwindigkeit $c_{T}$ nähert.

\subsubsection{Transkritische Strömung mit Schock}

Auch im Fall von transkritischen Strömungen mit Schock findet man Instabilität. Es wurden stabile Moden, ein nicht schwingender, monoton wachsender Mode gefunden, aber auch schwingende Moden, deren Amplitude im Lauf der Zeit zunimmt. Tabelle 3.7 zeigt einige der gefundenen Eigenwerte. Als Startwerte für $\omega$ wurde der Bereich mit $10^{-5} / \mathrm{sec}<\omega_{r}<0.2 / \mathrm{sec}$ und $10^{-4} / \mathrm{sec}<\left|\omega_{i}\right|<0.1 / \mathrm{sec}$ der komplexen Zahlenebene mit einer Schrittweite von

\footnotetext{
${ }^{7}$ Wenn die Größen $a, b$ und $c$ in Gleichung (3.8) reell sind, kann Instabilität nur auftreten, falls $\sqrt{b^{2}-a c}$ rein imaginär ist. $\omega$ setzt sich dann zusammen aus dem Realteil $\omega_{r}=b / a$ und dem Imaginärteil $\pm \sqrt{b^{2}-a c}$. Die beiden Lösungen von Gleichung (3.8) sind also für instabile Moden komplex konjugiert.
} 

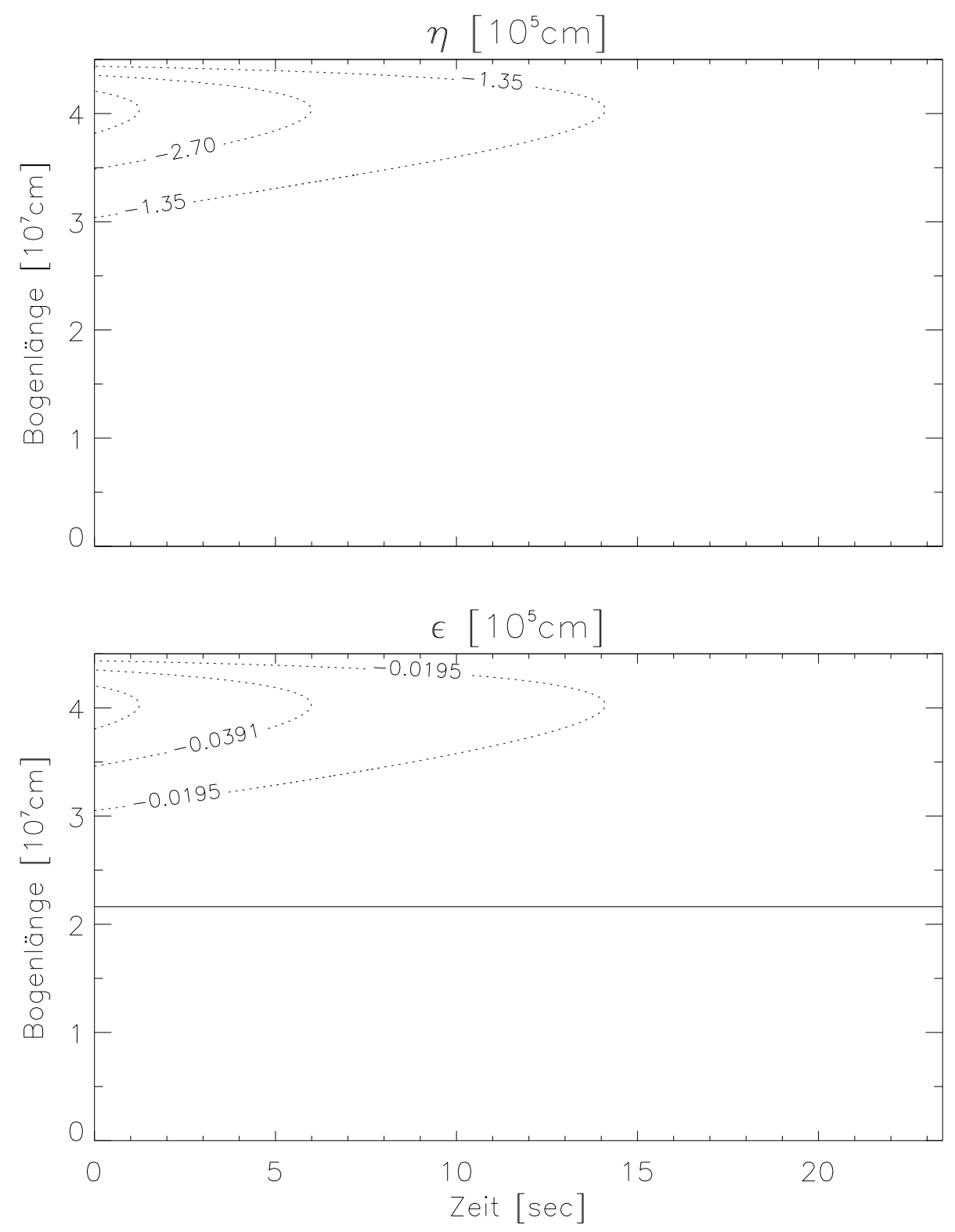

Abbildung 3.16: Konturlinien der Störung im Ort tangential (oben) und normal zur Flußröhre zum Eigenwert $\omega=\left(-6.68 \cdot 10^{-12}, 0.0854\right) / \mathrm{sec}$. Die Störungen gehören zum in Abbildung 2.4 gezeigten transkritischen Gleichgewicht ohne Schock. Es wurde bis hinter den kritischen Punkt integriert, der bei $s \approx 3.354 \cdot 10^{7} \mathrm{~cm}$ liegt. Der Mode zerfällt monoton. 

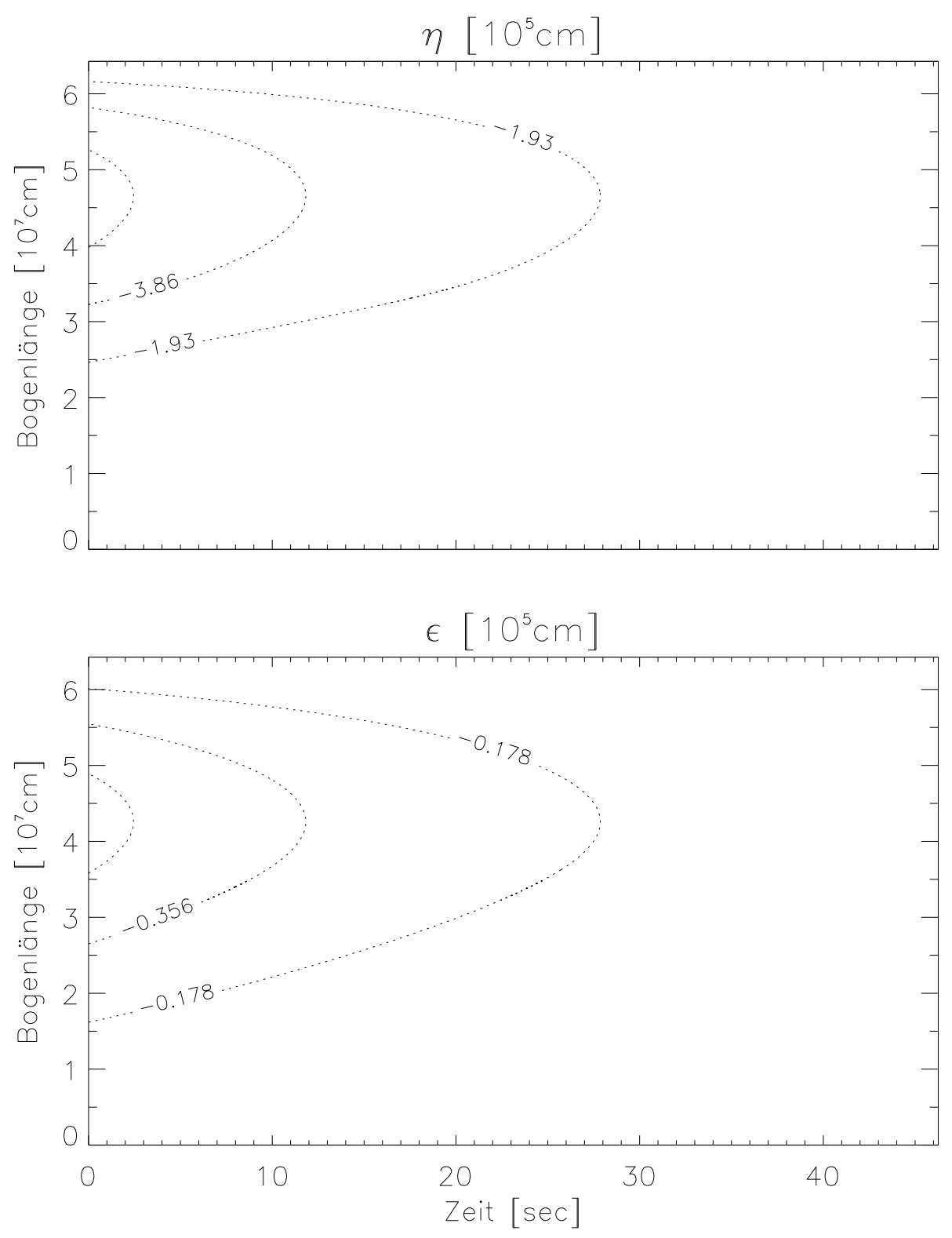

Abbildung 3.17: Konturlinien der Störung im Ort tangential (oben) und normal zur Flußröhre zum Eigenwert $\omega=\left(-3.37 \cdot 10^{-13}, 0.0432\right)$ /sec. Die Störungen gehören zum in Abbildung 2.4 gezeigten transkritischen Gleichgewicht ohne Schock. Es wurde bis zum rechten Fußpunkt der Röhre integriert. Der Mode zerfällt monoton. 


\begin{tabular}{|c|c|c|c|}
\hline$s_{1}\left[10^{7} \mathrm{~cm}\right]$ & $\omega[1 / \mathrm{sec}]$ & $\left(b^{2}-a c\right) / a^{2}\left[10^{-4} / \mathrm{sec}^{2}\right]$ & siehe Abb. \\
\hline \hline 1.5 & $\left(0.0990,-1.93 \cdot 10^{-9}\right)$ & 106 & 3.18 \\
\hline & $\left(0.197,1.36 \cdot 10^{-9}\right)$ & 422 & 3.19 \\
\hline & $\left(0.224,-6.62 \cdot 10^{-10}\right)$ & 509 & \\
\hline 2.0 & $\left(0.0701,-1.55 \cdot 10^{-10}\right)$ & 55.5 & \\
\hline & $\left(0.174,6.06 \cdot 10^{-10}\right)$ & 308 & \\
\hline 2.5 & $\left(0.0511,2.18 \cdot 10^{-9}\right)$ & 32.1 & \\
\hline & $\left(0.144,-4.48 \cdot 10^{-10}\right)$ & 214 & \\
\hline & $\left(0.154,1.07 \cdot 10^{-9}\right)$ & 294 & 3.20 \\
\hline 3.0 & $\left(0.0353,1.24 \cdot 10^{-9}\right)$ & 19.2 & \\
\hline & $\left(0.108,-2.88 \cdot 10^{-9}\right)$ & 185 & 3.21 \\
\hline & $\left(0.125,-8.55 \cdot 10^{-12}\right)$ & 163 & \\
\hline & $\left(0.144,-5.35 \cdot 10^{-9}\right)$ & 335 & \\
\hline & $\left(0.0142,1.31 \cdot 10^{-8}\right)$ & 17.6 & \\
\hline & $\left(0.0312,3.22 \cdot 10^{-9}\right)$ & 62.2 & \\
\hline & $\left(0.0474,3.95 \cdot 10^{-9}\right)$ & 138 & \\
\hline & $\left(0.0634,3.30 \cdot 10^{-9}\right)$ & 248 & \\
\hline
\end{tabular}

Tabelle 3.6: Eigenwerte $\omega$ aus Stabilitätsanalysen zu dem in Abbildung 2.4 dargestellten transkritischen Modell ohne Schock in Abhängigkeit von der Bogenlänge $s_{1}$, bis zu der die Integration erfolgte. Bei den hier angegebenen Eigenwerten wurde die Integration vor dem kritischen Punkt abgebrochen.

\begin{tabular}{|c|c|}
\hline$\omega[1 / \mathrm{sec}]$ & dargestellt in Abb. \\
\hline \hline$\left(8.82 \cdot 10^{-21},-0.0225\right)$ & 3.22 \\
\hline$\left(4.59 \cdot 10^{-21}, 0.0238\right)$ & 3.23 \\
\hline$(0.0467,-0.0958)$ & 3.24 \\
\hline$\left(0.0663,2.66 \cdot 10^{-4}\right)$ & \\
\hline$\left(0.116,-2.43 \cdot 10^{-6}\right)$ & 3.25 \\
\hline$\left(0.180,-1.03 \cdot 10^{-4}\right)$ & \\
\hline
\end{tabular}

Tabelle 3.7: Eigenwerte aus Stabilitätsrechnungen zu transkritischen Gleichgewichten mit Schock 

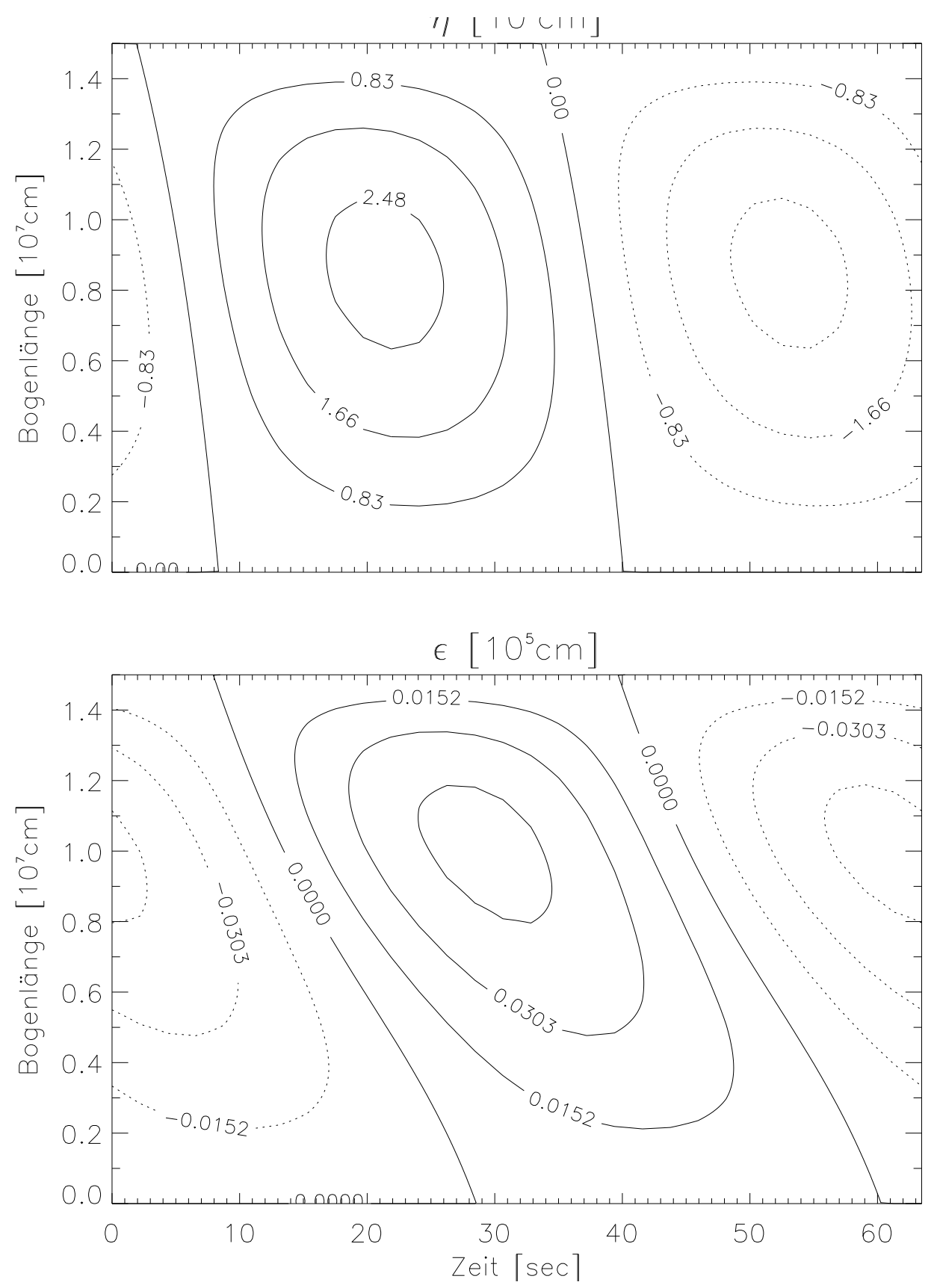

Abbildung 3.18: Konturlinien der Störung im Ort tangential (oben) und normal zur Flußröhre zum Eigenwert $\omega=\left(0.0990,-1.93 \cdot 10^{-9}\right) /$ sec. Die Störungen gehören zum in Abbildung 2.4 gezeigten transkritischen Gleichgewicht ohne Schock. Die Integration wurde weit vor dem kritischen Punkt abgebrochen. 

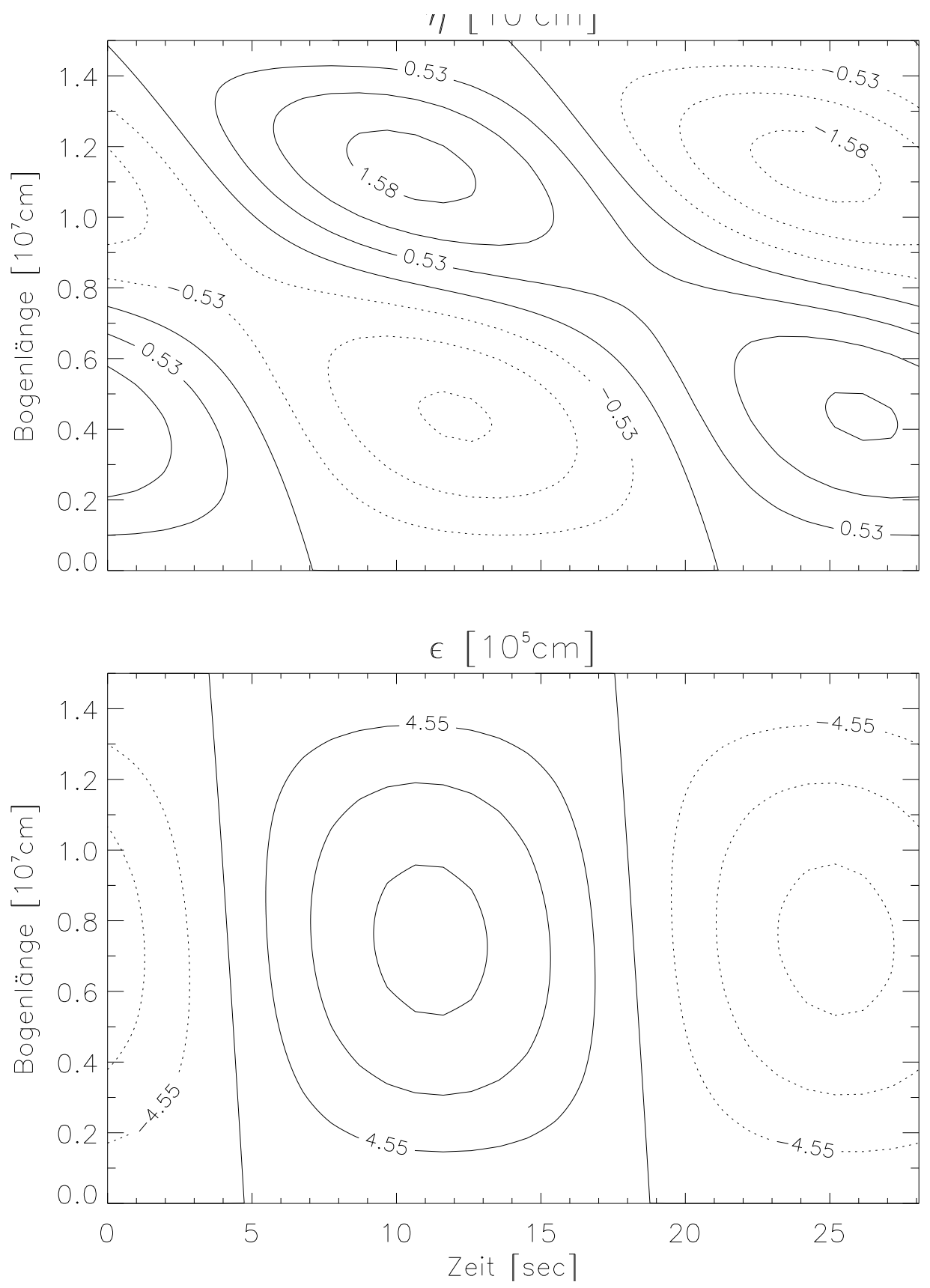

Abbildung 3.19: Konturlinien der Störung im Ort tangential (oben) und normal zur Flußröhre zum Eigenwert $\omega=\left(0.224,-6.62 \cdot 10^{-10}\right)$ /sec. Die Störungen gehören zum in Abbildung 2.4 gezeigten transkritischen Gleichgewicht ohne Schock. Die Integration wurde weit vor dem kritischen Punkt abgebrochen. 

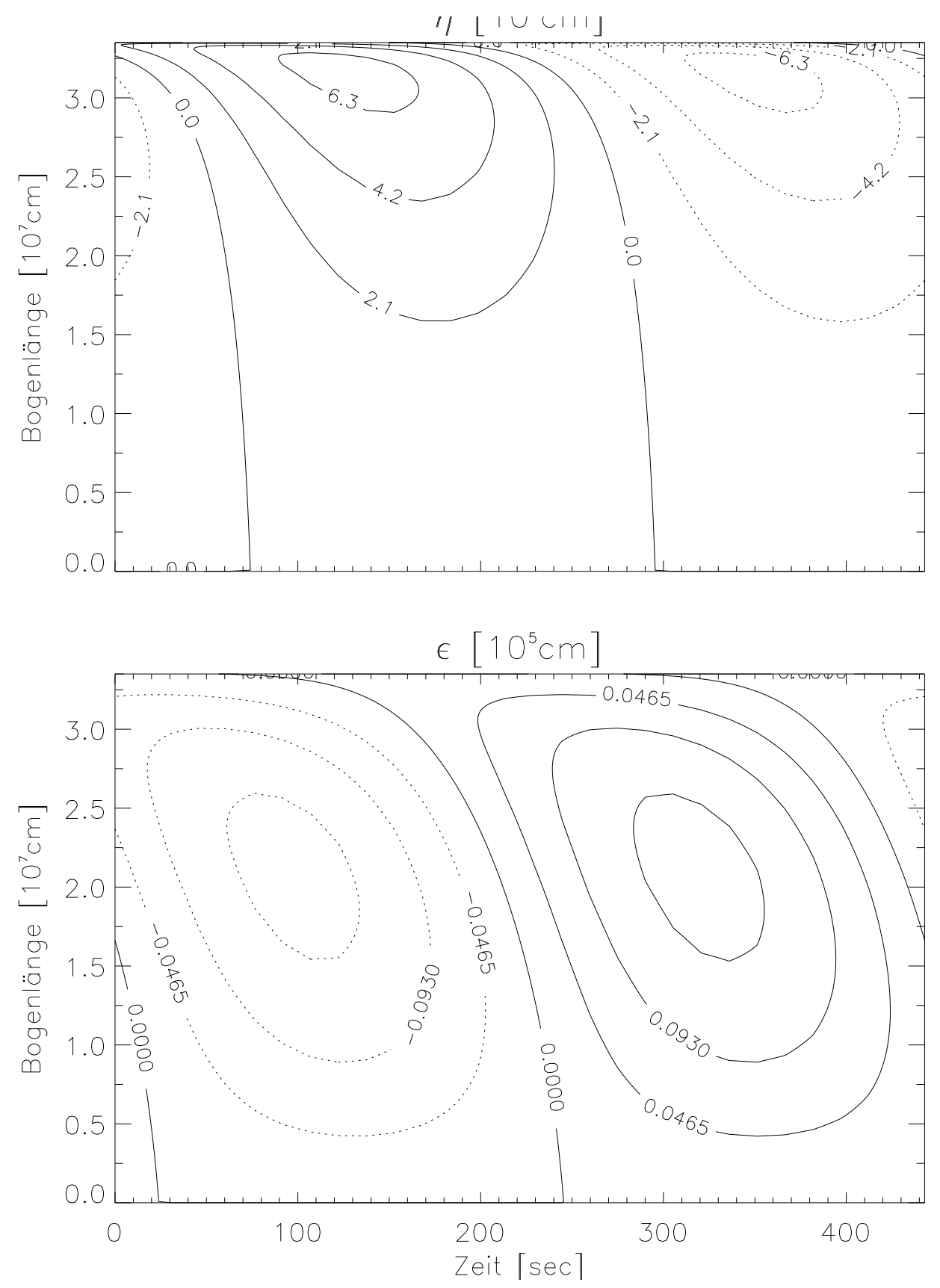

Abbildung 3.20: Konturlinien der Störung im Ort tangential (oben) und normal zur Flußröhre zum Eigenwert $\omega=\left(0.0142,1.31 \cdot 10^{-8}\right) / \mathrm{sec}$. Die Störungen gehören zum in Abbildung 2.4 gezeigten transkritischen Gleichgewicht ohne Schock. Die Integration wurde kurz vor dem kritischen Punkt abgebrochen. Mit zunehmender Annäherung an den kritischen Punkt verläuft $\eta$ steiler. 

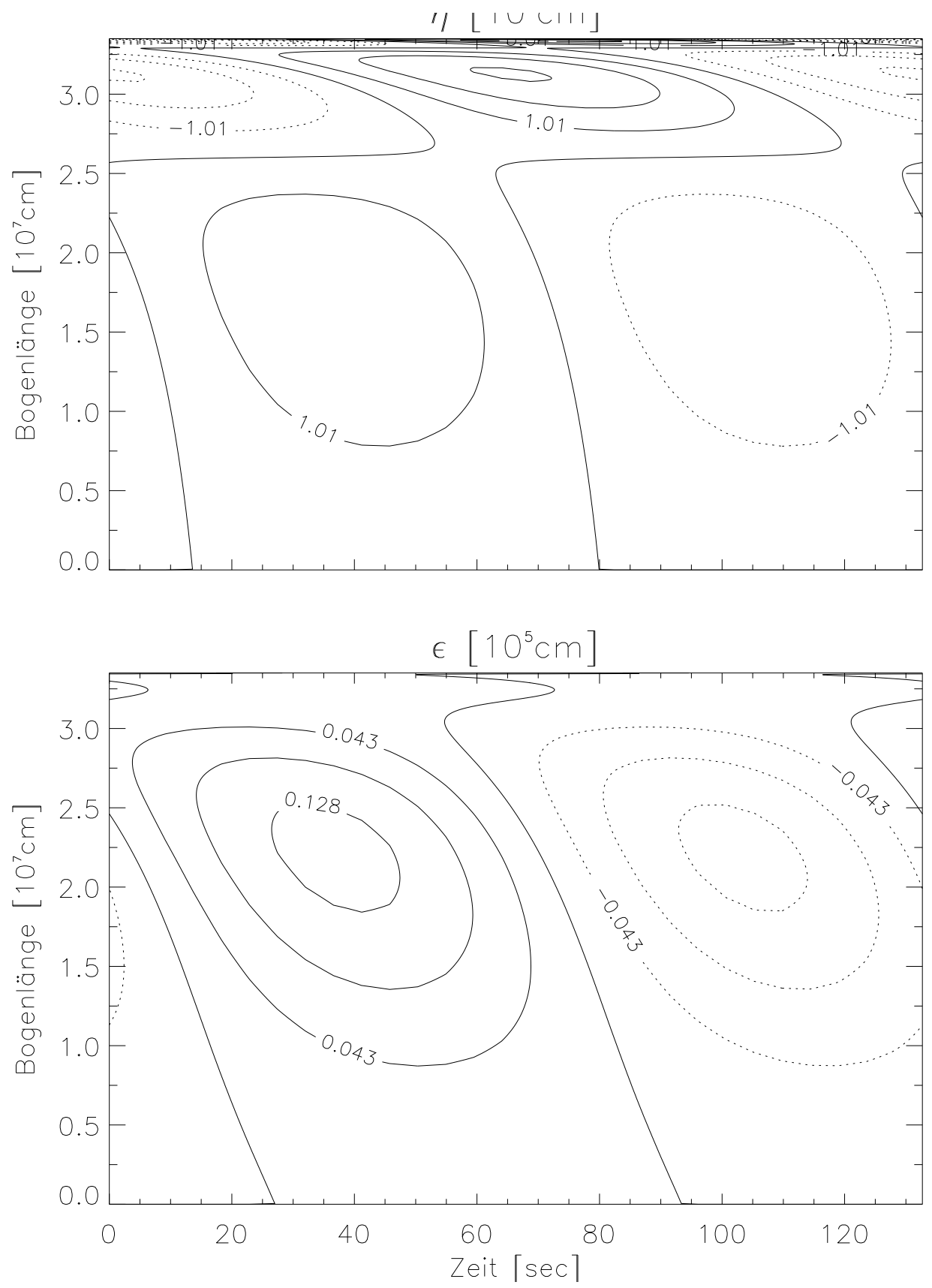

Abbildung 3.21: Konturlinien der Störung im Ort tangential (oben) und normal zur Flußröhre zum Eigenwert $\omega=\left(0.0474,3.95 \cdot 10^{-9}\right) / \mathrm{sec}$. Die Störungen gehören zum in Abbildung 2.4 gezeigten transkritischen Gleichgewicht ohne Schock. Die Integration wurde kurz vor dem kritischen Punkt abgebrochen. Wieder findet sich der steile Verlauf von $\eta$ in der Nähe des kritischen Punkts. 
$10^{-3} /$ sec für Real- und Imaginärteil durchsucht. Die tangentiale Komponente der Ortsstörungen zu den Eigenwerten mit den größten Anwachsraten hat an den Fußpunkten der Flußröhre ihre größte Auslenkung's Diese Auslenkung an den Fußpunkten wird erst durch die Regularitätsforderung an den kritischen Punkten möglich. Es ist nicht auszuschließen, daß die gefundenen Instabilitäten durch die Aufgabe der Randbedingungen $\tilde{\eta}=0$ an den Grenzen des Integrationsgebiets ermöglicht werden. Die Anwachs- bzw. Zerfallsraten liegen im Bereich von $10^{-4} / \mathrm{sec}$ für anwachsende bzw. zerfallende Lösungen der Stabilitätsgleichungen, die nicht an den Integrationsrändern ihre stärkste Auslenkung haben und variieren von $10^{-2}$ bis $10^{-1} /$ sec für die problematischen Lösungen mit den großen Amplituden an den Rändern.

\subsubsection{Superkritische Strömungen}

Als Startwerte wurden Eigenwerte im Intervall $10^{-5} / \mathrm{sec}<\omega_{r}<0.05 / \mathrm{sec}$ und $10^{-4} / \mathrm{sec}<$ $\left|\omega_{i}\right|<0.01 / \mathrm{sec}$ vorgegeben. Alle gerechneten Stabilitätsanalysen überkritischer Strömungen weisen instabile Moden auf. Es wurden stabile schwingende und instabile schwingende Moden gefunden. Tabelle 3.8 gibt einige der gefundenen Eigenwerte sowie den die Stabilität beschreibenden Term $\left(b^{2}-a c\right) / a^{2}$ aus dem globalen Kriterium 3.11 an. Wie schon bei den transkritischen Strömungen ohne Schock findet man mit Hilfe des globalen Kriteriums bei Eigenwerten, deren Imaginärteil nicht verschwindet, unabhängig vom Vorzeichen des Imaginärteils Instabilität. Auch bei den hier angegebenen Fällen ist bei nicht verschwindendem Imaginärteil von $\omega$ nach Gleichung (3.8) wieder $\omega^{*}$ Eigenwert, was die Instabilität nach dem globalen Kriterium erklärt.

Vergleicht man die zu stabilen Moden gehörigen Ortsstörungen in den Abbildungen 3.26 und 3.27 mit denen der instabilen Mode in Abbildung 3.28, so fällt auf, daß die Symmetrie der Amplituden zum Röhrenscheitelpunkt bei den instabilen Moden wegfällt. Anders als bei den unterkritischen Strömungen breiten sich die tangentialen Ortsstörungen der Moden mit den kleinsten positiven $\omega_{r}$ in Strömungsrichtung aus. Die Ausbreitungsrichtung der zugehörigen normalen Ortsstörungen ist schon nicht mehr für alle Gleichgewichte gleich. Auch bei Stabilitätsuntersuchungen überkritischer Modelle zeigt sich ein zunehmend steiler Verlauf der tangentialen Ortsstörung $\eta$ in der Umgebung des Röhrenscheitelpunkts, je mehr sich die Strömungsgeschwindigkeit $v$ der kritischen Geschwindigkeit $c_{T}$ nähert.

${ }^{8}$ siehe Abbildungen 3.22 und 3.24 . 

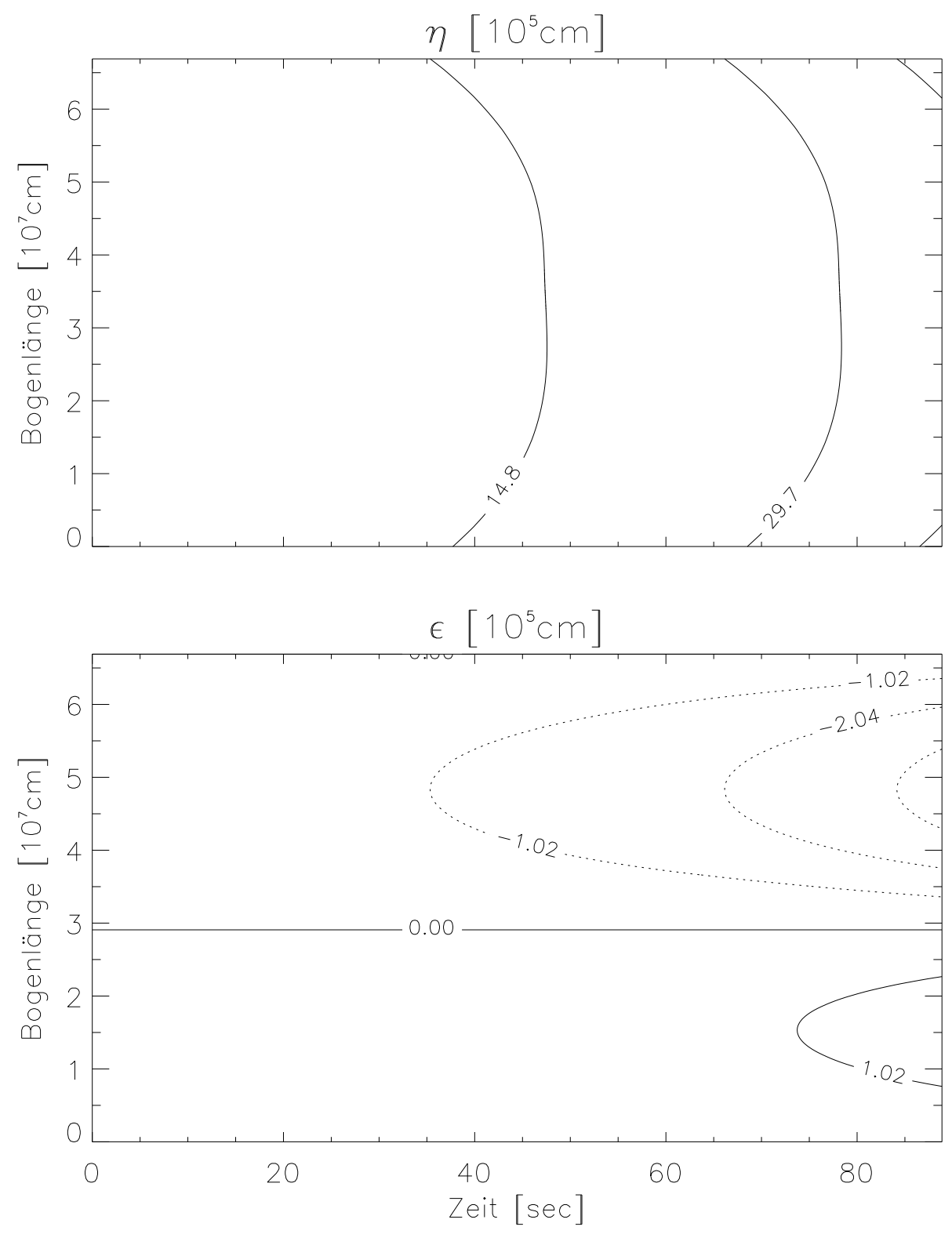

Abbildung 3.22: Konturlinien der Störungen im Ort tangential (oben) und normal zur Flußröhre zum Eigenwert $\omega=\left(8.82 \cdot 10^{-21},-0.0225\right) /$ sec. Die Störungen gehören zum in Abbildung 2.5 gezeigten transkritischen Gleichgewicht mit Schock. Im oberen Teil der Abbildung wird deutlich, daß die tangentiale Ortsstörung an den Fußpunkten nicht verschwindet. Dies ist die Folge der gestellten Regularitätsbedingungen. Der kritische Punkt liegt bei $s \approx 3.354 \cdot 10^{7} \mathrm{~cm}$, der Schock bei $s \approx 3.679 \cdot 10^{7} \mathrm{~cm}$. Der Mode wächst monoton an. 

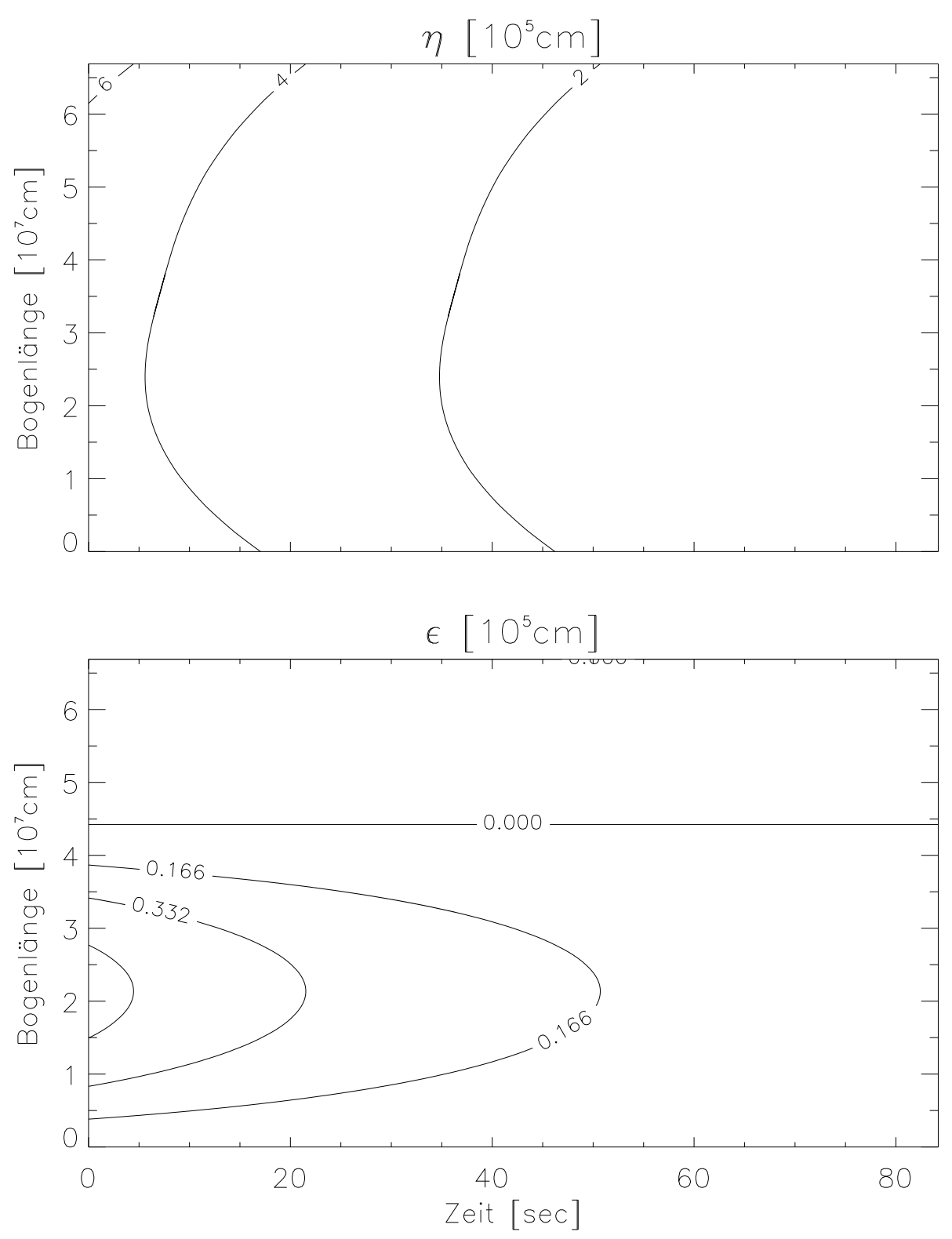

Abbildung 3.23: Konturlinien der Störungen im Ort tangential (oben) und normal zur Flußröhre zum Eigenwert $\omega=\left(4.59 \cdot 10^{-21}, 0.0238\right) /$ sec. Die Störungen gehören zum in Abbildung 2.5 gezeigten transkritischen Gleichgewicht mit Schock. Der Mode zerfällt monoton. 

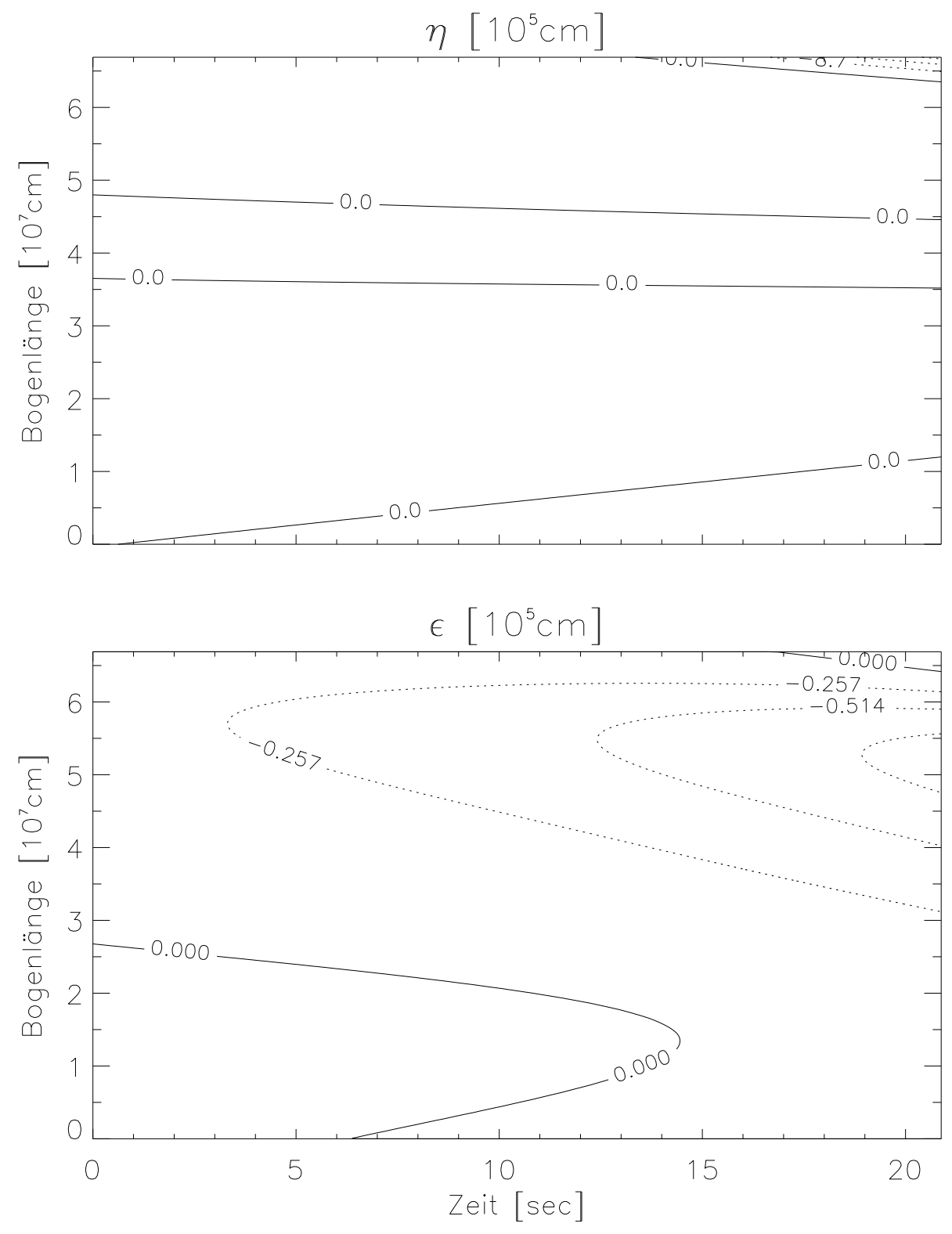

Abbildung 3.24: Konturlinien der Störungen im Ort tangential (oben) und normal zur Flußröhre zum Eigenwert $\omega=(0.0467,-0.0958)$ /sec. Die Störungen gehören zum in Abbildung 2.5 gezeigten transkritischen Gleichgewicht mit Schock. Dieser Mode ist der mit der größten gefundenen Anwachsrate. 

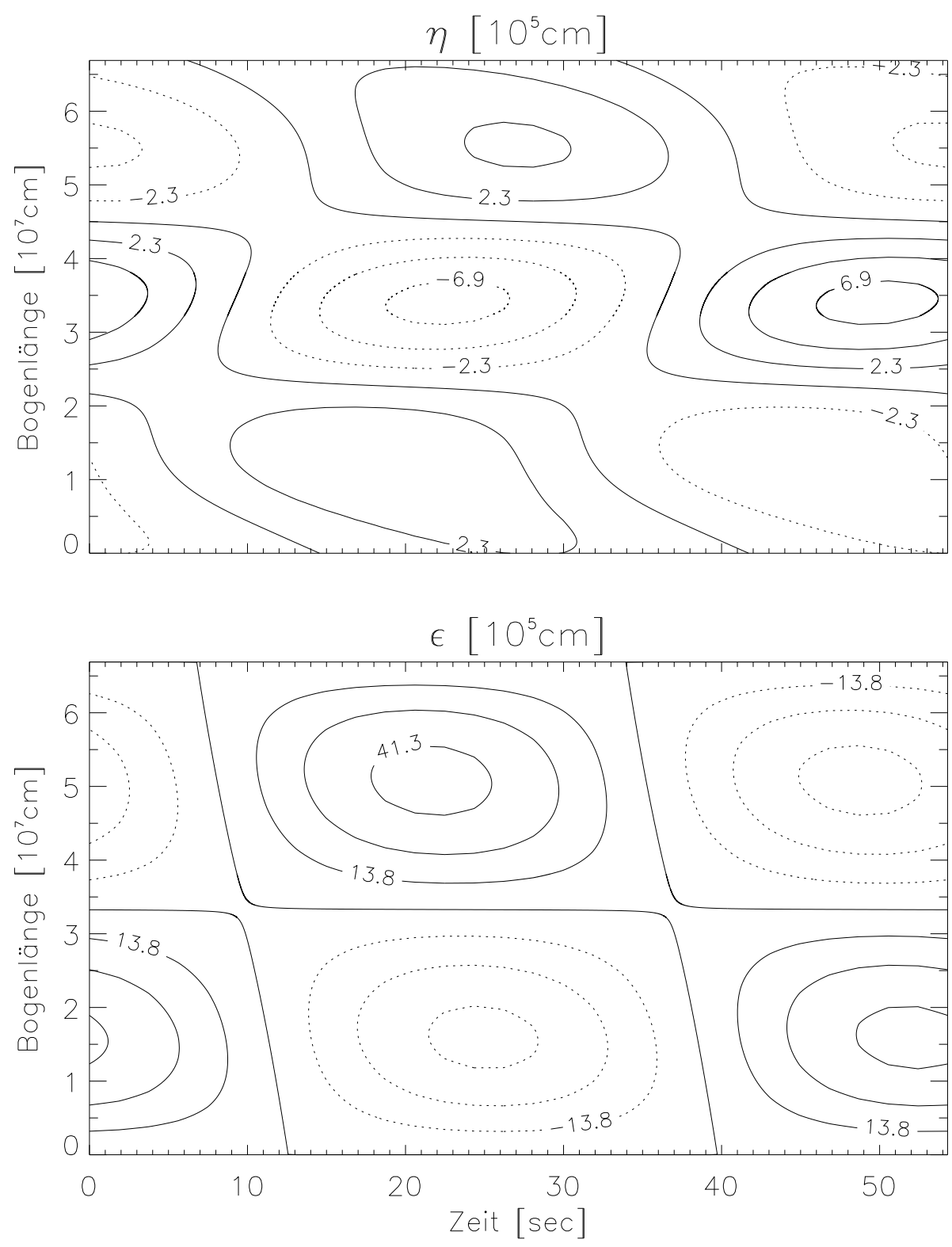

Abbildung 3.25: Konturlinien der Störungen im Ort tangential (oben) und normal zur Flußröhre zum Eigenwert $\omega=\left(0.116,-2.43 \cdot 10^{-6}\right) /$ sec. Die Störungen gehören zum in Abbildung 2.5 gezeigten transkritischen Gleichgewicht mit Schock. Dieser Mode ist im Rahmen numerischer Genauigkeit nach dem Kriterium aus Abschnitt 3.4.1 stabil. 


\begin{tabular}{|c|c|c|c|}
\hline$v_{0}[\mathrm{~cm} / \mathrm{sec}]$ & $\omega[1 / \mathrm{sec}]$ & $\left(b^{2}-a c\right) / a^{2}\left[10^{-4} / \mathrm{sec}^{2}\right]$ & siehe Abb. \\
\hline \hline 781483 & $\left(0.00506,-6.10 \cdot 10^{-10}\right)$ & $3.10 \cdot 10^{3}$ & 3.26 \\
\hline & $\left(0.0136,-2.24 \cdot 10^{-9}\right)$ & 970 & \\
\hline & $\left(0.0177,-2.99 \cdot 10^{-9}\right)$ & 915 & \\
\hline & $\left(0.0218,-3.01 \cdot 10^{-9}\right)$ & 842 & \\
\hline & $\left(0.0260,-3.18 \cdot 10^{-9}\right)$ & 333 & \\
\hline & $(0.0297,-0.00171)$ & -0.0318 & \\
\hline & $(0.0297,0.00171)$ & -0.0317 & \\
\hline & $\left(0.0144,-3.66 \cdot 10^{-9}\right)$ & 141 & \\
\hline & $\left(0.0258,-4.42 \cdot 10^{-9}\right)$ & 39.6 & \\
\hline & $\left(0.0301,-2.64 \cdot 10^{-10}\right)$ & 4.94 & 3.27 \\
\hline & $\left(0.0341,-1.10 \cdot 10^{-10}\right)$ & 29.4 & 3.28 \\
\hline & $\left(0.0470,3.95 \cdot 10^{-9}\right)$ & 142 & 3.29 \\
\hline & $(0.0541,-0.00152)$ & -0.0218 & \\
\hline & $(0.0541,0.00152)$ & -0.0218 & \\
\hline & $\left(0.0172,-3.98 \cdot 10^{-9}\right)$ & 98.2 & \\
\hline & $(0.0299,-0.00281)$ & -0.0790 & \\
\hline & $(0.0299,0.00281)$ & -0.0790 & 134 \\
\hline & $\left(0.0423,-5.39 \cdot 10^{-10}\right)$ & -0.105 & \\
\hline & $(0.0540,-0.00324)$ & -0.105 & \\
\hline & $(0.0540,0.00324)$ & & \\
\hline
\end{tabular}

Tabelle 3.8: Eigenwerte aus Stabilitätsrechnungen zu überkritischen Gleichgewichten. Bei den nach dem globalen Kriterium instabilen Moden sind jeweils der anwachsende und der zerfallende Mode getrennt angegeben, da sich die zugehörigen Eigenfunktionen unterscheiden, wie aus den Abbildungen 3.28 und 3.29 entnommen werden kann. 

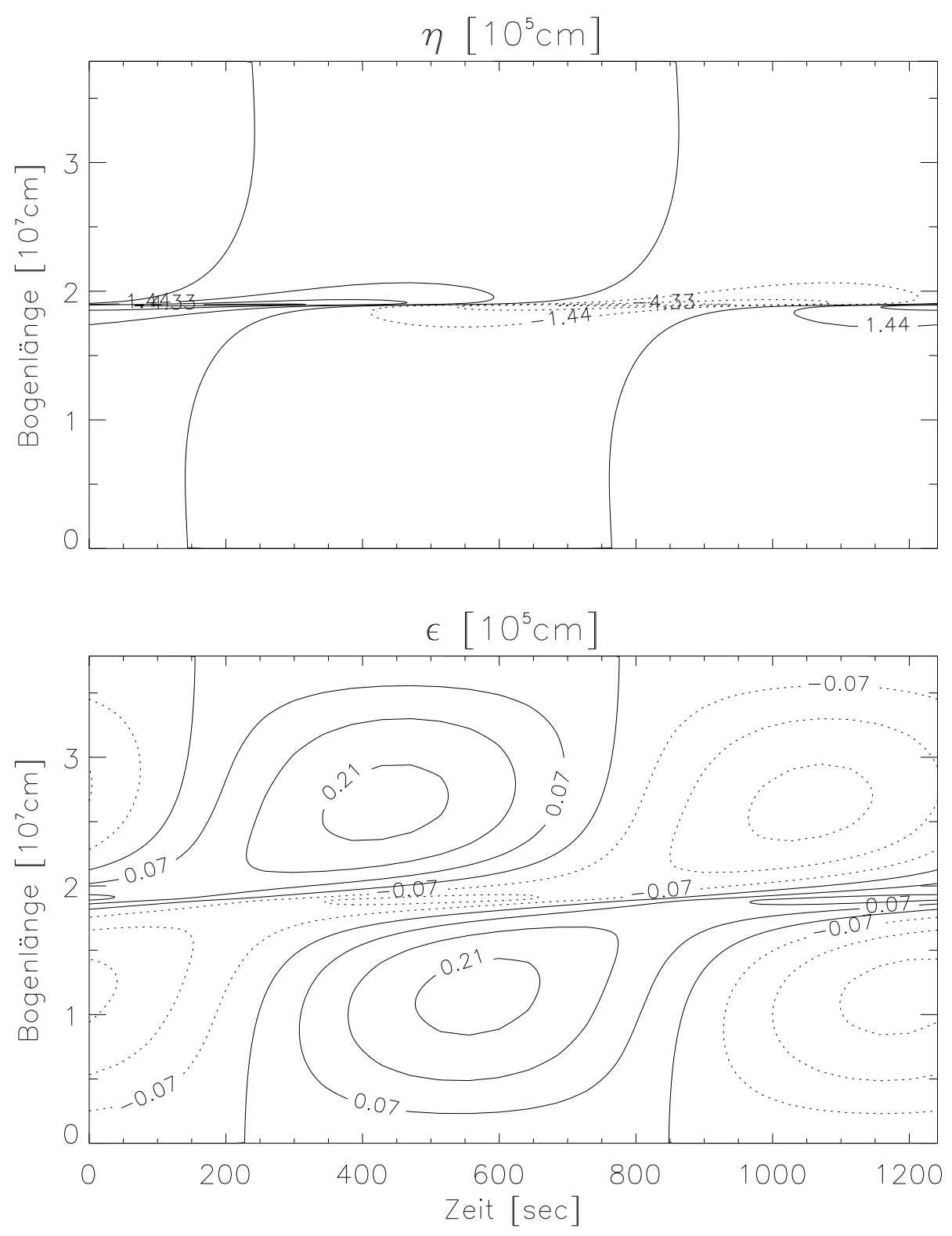

Abbildung 3.26: Konturlinien der Störungen im Ort tangential (oben) und normal zur Flußröhre zum Eigenwert $\omega=\left(0.00506,-6.10 \cdot 10^{-10}\right) / \mathrm{sec}$. Die Störungen gehören zum in Abbildung 2.6 gezeigten überkritischen Gleichgewicht. Man beachte den steilen Verlauf von $\eta$ in der Umgebung des Scheitelpunktes der Flußröhre. 

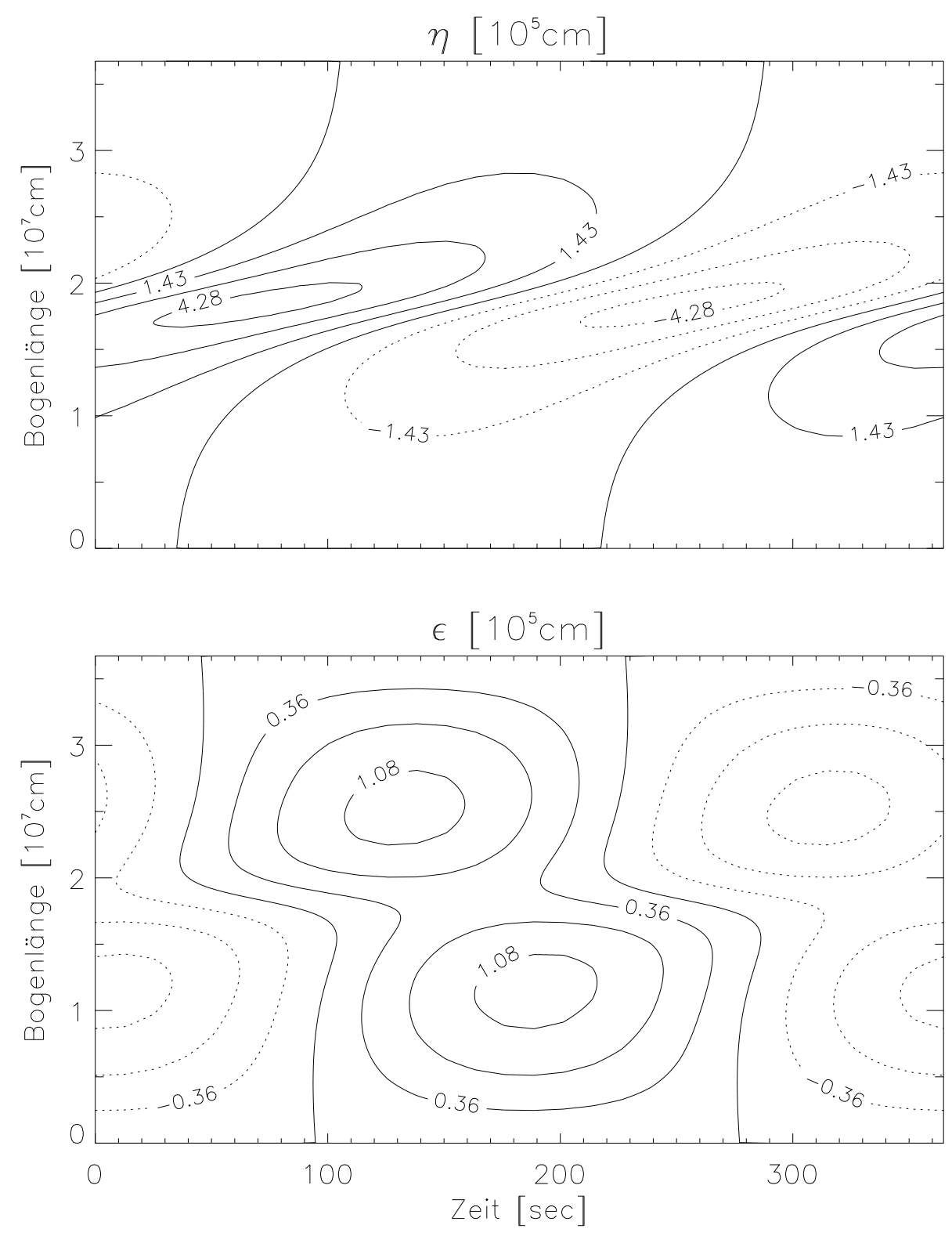

Abbildung 3.27: Konturlinien der Störungen im Ort tangential (oben) und normal zur Flußröhre zum Eigenwert $\omega=\left(0.0172,-3.98 \cdot 10^{-9}\right) /$ sec. Die Störungen gehören zum in Abbildung 2.7 gezeigten überkritischen Gleichgewicht. 

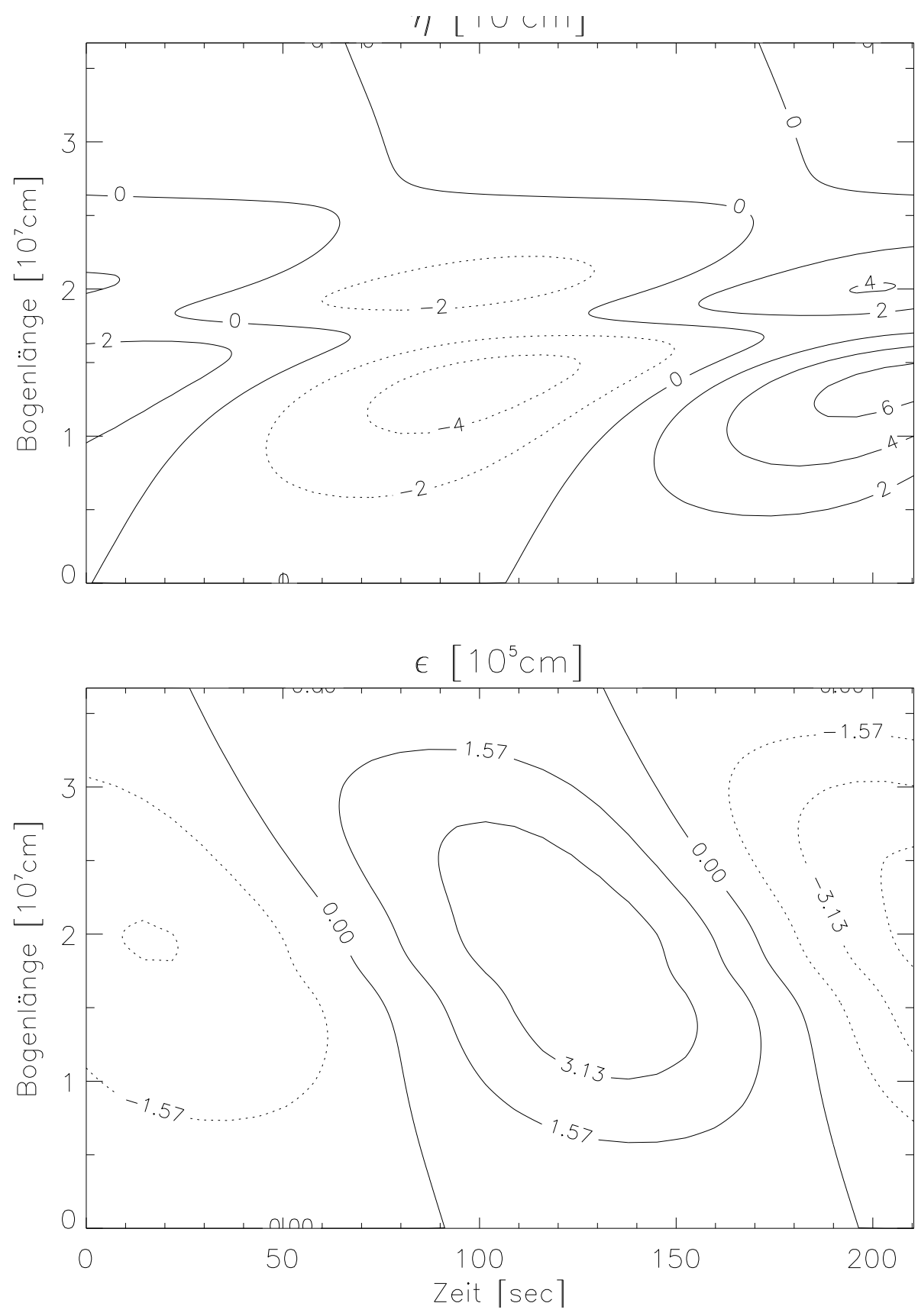

Abbildung 3.28: Konturlinien der Störungen im Ort tangential (oben) und normal zur Flußröhre zum Eigenwert $\omega=(0.0299,-0.00281)$ /sec. Die Störungen gehören zum in Abbildung 2.7 gezeigten überkritischen Gleichgewicht. Die Amplitude der Ortsstörungen wächst im Lauf der Zeit an. Der Mode ist instabil. Die tangentialen Ortsstörungen mit größeren Auslenkungen finden sich im linken Teil der Flußröhre. 

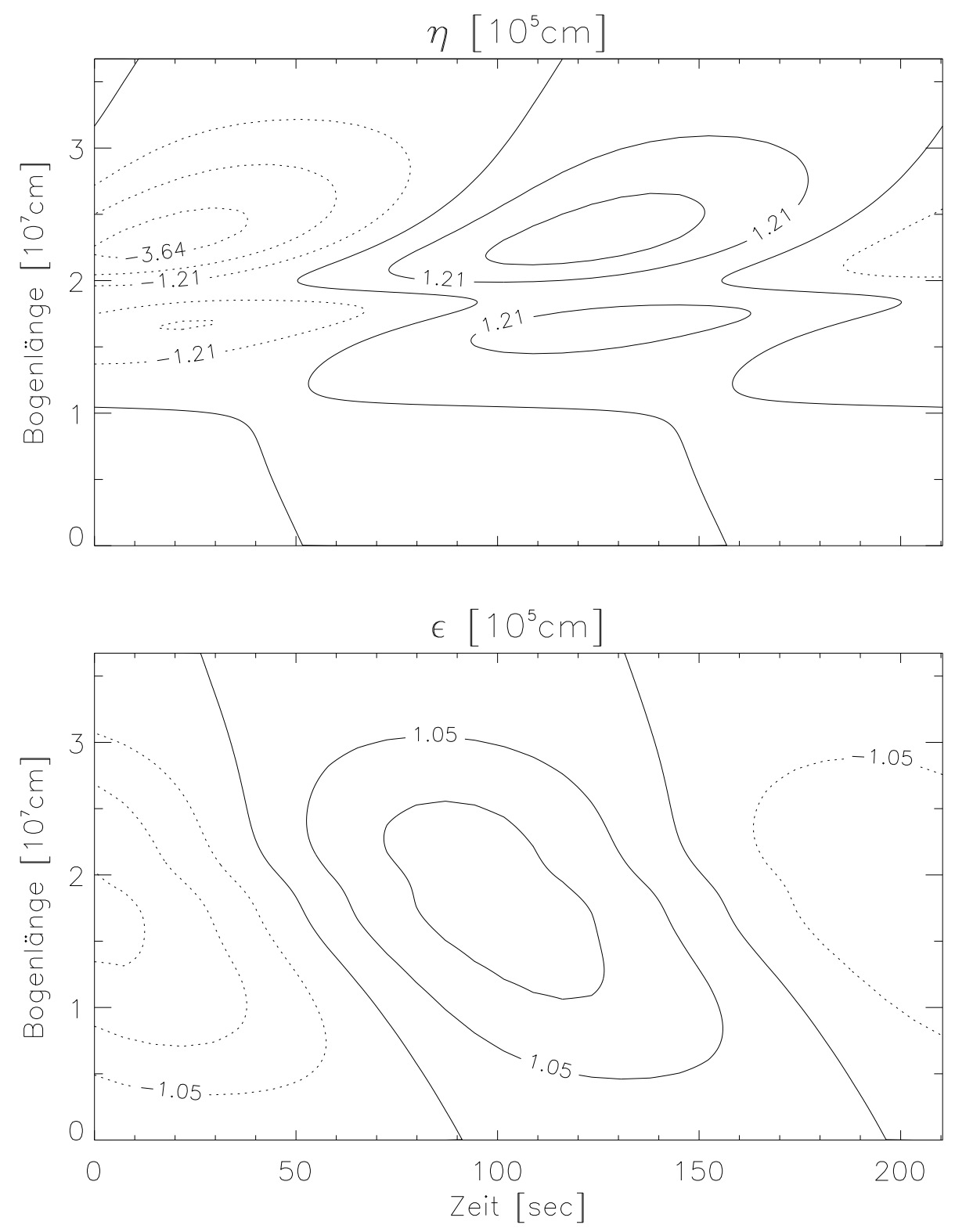

Abbildung 3.29: Konturlinien der Störungen im Ort tangential (oben) und normal zur Flußröhre zum Eigenwert $\omega=(0.0299,0.00281)$ /sec. Die Störungen gehören zum in Abbildung 2.7 gezeigten überkritischen Gleichgewicht. Die Amplitude der Ortsstörungen bei diesem Mode zerfallen mit der Zeit, wobei die größten Amplituden der tangentialen Ortsstörung im rechten Teil der Röhre erreicht werden.. 


\section{Zusammenfassung und Diskussion}

In dieser Arbeit wurde nach dem Vorbild von Thomas und Montesinos (1991) ein einfaches stationäres Modell für eine Siphonströmung in der Sonnenfleckenpenumbra erstellt. Dieses Modell wurde dann mit Hilfe des Formalismus' von Schmitt (1995; 1998) auf Stabilität gegenüber kleinen adiabatischen Störungen untersucht. Es wurden unter- und überkritische sowie transkritische Modelle ohne und mit Schock betrachtet.

Sowohl bei unter- als auch bei überkritischen Modellen fand sich mit zunehmender Annäherung der Strömungsgeschwindigkeit am Flußröhrenscheitelpunkt an die kritische Geschwindigkeit ein immer steilerer Verlauf der Ortsstörung entlang der Flußröhre in der Umgebung des Scheitelpunkts. Dies machte in dem numerischen Programm zur Lösung des Differentialgleichungssystems 3.4 die Verwendung von nicht-äquidistanten Gittern erforderlich. Um im Fall sehr kleiner Differenz zwischen Strömungsgeschwindigkeit und kritischer Geschwindigkeit, wie z.B. bei transkritischen Strömungen in der Umgebung des Röhrenscheitelpunkts, numerische Instabilität zu vermeiden, wurden bei der Integration Methoden für steife Differentialgleichungen verwendet.

In Bezug auf die Stabilität der untersuchten Gleichgewichtslösungen läßt sich als Ergebnis festhalten:

- Rein unterkritische Strömungen sind stabil.

- Transkritische Strömungen ohne Schock sind instabil. Es wurde in allen untersuchten Fällen ein nicht schwingender, monoton anwachsender Mode gefunden.

- Bei transkritischen Strömungen mit Schock stößt man auf Schwierigkeiten bei der Lösung der beschreibenden Differentialgleichungen. Reguläre Lösungen wurden nur unter Aufgabe von Randbedingungen zugunsten von Regularitätsbedingungen an den beiden kritischen Punkten erhalten. Neben stabilen Moden wurden in allen untersuchten Fällen nicht schwingende, monoton wachsende und schwingende anwachsende Moden gefunden.

- Rein überkritsche Strömungen sind instabil. Bei allen untersuchten Modellen wurden außer stabilen auch schwingende, im Verlauf der Zeit anwachsende Moden gefunden.

Schmitt (1995; 1998) hat das Gleichgewichtsmodell von Degenhardt (1990) bei unterkritischen Strömungsgeschwindigkeiten auf Stabilität hin untersucht und Instabilität für wachsende Strömungsgeschwindigkeit oder schwächer werdendes Magnetfeld im linken Fußpunkt der Flußröhre gefunden. Diese Instabilität hat er als „konvektive Instabilität“ gedeutet. Bei dem 
Degenhardtschen Modell waren die Flußröhren in eine aus zwei Teilen bestehende Atmosphäre eingebettet, der untere Teil dieser Atmosphäre war instabil geschichtet. Da in der vorliegenden Arbeit das die Flußröhre umgebende Medium stabil geschichtet ist, war zu erwarten, daß keine durch Konvektion bedingte Instabilität auftritt. Der Vergleich mit den Stabilitätsuntersuchungen unterkritischer Modelle hier bestätigt damit, daß die von Schmitt gefundene Instabilität konvektiver Natur ist.

Bei mit nennenswerter Geschwindigkeit durchströmten Gleichgewichtsmodellen findet man im Fall unterkritischer Strömungen bei den Grundmoden eine Ausbreitung der Ortsstörungen entgegen der Strömungsrichtung, die Störungen stellen dabei keine ebene Welle dar. Es handelt sich vielmehr um ein Wellenpaket, welches sich in der rechten Hälfte der Röhre bildet, im Lauf der Zeit mit anwachsender Amplitude in den linken Teil der Röhre wandert und dort verschwindet. Im Fall von mit überkritischer Geschwindigkeit durchströmten Modellen kehrt sich die Ausbreitungsrichtung der Störung um und die obige Beschreibung des Werdens und Vergehens des Wellenpakets trifft für die gefundenen Ortsstörungen normal zur Röhre nicht mehr zu. Eine Identifikation dieser wellenförmigen Ausbreitung mit einer der typischen MHD-Wellen ist nicht möglich, auch wenn die Umkehr der Ausbreitungsrichtung der tangentialen Ortsstörung beim Übergang von Strömungen mit überall $v<c_{T}$ zu Modellen mit überall $v>c_{T}$ den langsamen magnetoakustischen Mode nahe legt.

Transkritische Strömungen ohne Schock sowie die überkritischen Modelle treten in der Realität bei den betrachteten Strömungen in Sonnenfleckenpenumbren nicht auf. Die Werte, die in diesen Fällen für die beobachtbaren Größen in einem oder beiden Röhrenfußpunkten gälten, werden nicht gemessen. Die Untersuchung solcher Modelle soll aufzeigen, daß die gefundenen Instabilitäten immer mit dem Auftreten von Strömungsgeschwindigkeiten größer der kritischen Geschwindigkeit $c_{T}$ verbunden sind. Im besonderen ist ein transkritisches Modell ohne Schock erstellt worden, bei dem nur Teile der Flußröhre, immer beginnend am linken Fußpunkt, auf Stabilität hin untersucht wurden. Alle Teilmodelle, bei denen die Strömungsgeschwindigkeit an jedem Punkt unterkritisch ist, sind stabil. Sobald aber ein auch nur kleiner Bereich mit überkritischer Strömungsgeschwindigkeit im Teilmodell enthalten ist, wurde Instabilität gefunden. Die Untersuchungen des transkritischen Modells ohne Schock legen also nahe, daß mit dem Überschreiten der kritischen Geschwindigkeit $c_{T}$ am kritischen Punkt eine Instabilität auftritt.

Bei transkritischen Modellen mit Schock mußten, um reguläre Lösungen zu finden, Randbedingungen aufgegeben werden. Die erhaltenen Lösungen beinhalten für jedes untersuchte Gleichgewicht $[$ instabile Moden. Die Möglichkeit, daß die gefundene Instabilität durch das Lockern der Randbedingungen verursacht wurde, ließ sich nicht ausschließen. Durch die Untersuchung der überkritischen und Teilmodelle konnte aber gezeigt werden, daß ein Überschreiten der kritischen Strömungsgeschwindigkeit immer mit dem Auftreten von Instabilitäten verbunden ist. Es ist also, unabhängig von der Lockerung der Randbedingungen, zu erwarten, daß die transkritischen Modelle mit Schock instabil sind.

\footnotetext{
${ }^{1}$ In dieser Arbeit wird nur ein transkritisches Gleichgewicht mit Schock präsentiert.
} 


\section{Erweiterungsmöglichkeiten und Ausblick}

Eine weitere Möglichkeit sich mit der Stabilität der erstellten Gleichgewichtsmodelle zu befassen wäre, die Zeitabhängigkeit der Stabilitätsgleichungen nicht mittels eines Exponentialansatzes zu behandeln sondern ein numerisches Programm zu entwickeln, das Zeit- und Ortsabhängigkeit der Gleichungen behandelt. Dies hieße, die Stabilitätsanalyse als ein Anfangswertproblem anzugehen. Es ist wahrscheinlich, daß sich bei einer solchen Untersuchung die bei der Analyse transkritischer Gleichgewichte mit Schock auftretenden Sprünge nicht in endlicher Zeit entwickelten. In diesem Fall müßte nicht zu den in dieser Arbeit verwendeten indirekten Methoden gegriffen werden, um eine Instabilität nachzuweisen.

Auch könnte man davon abgehen, ein Gleichgewichtsmodell zu konstruieren und es dann auf seine Stabilität gegenüber kleinen Störungen zu untersuchen. Als Alternative könnte ein raum- und zeitabhängiges Modell einer Flußröhre erstellt werden. Es müßten dann allerdings die nichtlinearen Gleichungen der MHD zeit- und ortsabhängig gelöst werden, was noch immer ein beachtliches numerisches Problem darstellt.

Sowohl im Gleichgewichtsmodell wie auch in der Stabilitätsanalyse wurde eine Dissipation von Energie nach außen nicht berücksichtigt. Gerade bei Strömungen mit hoher Strömungsgeschwindigkeit sollte aber Dissipation eine Rolle spielen. Auch bei einem Schock ist sie von Bedeutung. Es ist zu erwarten, daß mit Berücksichtigung von Dissipation die Ortsstörungen in den kritischen Punkten regulär bleiben.

\section{Vergleich mit Beobachtungen}

Der aus der Beobachtung stammende Wert für die Lebensdauer penumbraler Filamente ist ungefähr eine halbe Stunde oder $\approx 2000$ sec. Die Anwachsraten, die in dieser Arbeit gefunden wurden liegen für (unrealistische) transkritische Strömungen ohne Schock bei $10^{-2}$ bis $10^{-1} / \mathrm{sec}$, diese Strömungen sind damit neben den im Gleichgewicht gefundenen zu geringen Werten für Druck und Dichte in der Flußröhre auch aufgrund ihrer Instabilität in Sonnenfleckenpenumbren nicht zu erwarten. Bei den (ebenfalls unrealistischen) superkritischen Strömungen liegen die Anwachsraten zwischen $10^{-3}$ und $3 \cdot 10^{-3} / \mathrm{sec}$. Auch dieser Wert liefert einen weiteren Grund dafür, daß solche Strömungen nicht in Sonnenfleckenpenumbren gefunden werden. Bei den Stabilitätsuntersuchungen transkritischer Gleichgewichte mit Schock fanden sich Anwachsraten von $10^{-4}$ bis $10^{-1} / \mathrm{sec}$, auch solche Strömungen sollten in der Realität keine Rolle spielen. Die Resultate letzterer Analysen sollte man allerdings mit Vorsicht betrachten, da zu ihrem Erhalt spezielle Annahmen gemacht wurden.

Der zur Zeit für wahrscheinlich gehaltene Durchmesser von Flußröhren in der Photosphäre liegt bei unter 100 km (Denker, 1998; Sütterlin und Wiehr, 1998) E. Das ist unterhalb des Auflösungsvermögens, welches man mit den vorhandenen Teleskopen unter Anwendung von Bildrekonstruktionsmethoden wie „Speckle-Rekonstruktion“ oder „Phase-Diversity“ erreichen kann. Mit dem Vakuum-Turm-Teleskop auf Teneriffa beobachtete Daten, die bei sehr guten Beobachtungsbedingungen gewonnen wurden, haben nach der Rekonstruktion mit den

\footnotetext{
${ }^{2}$ Es gibt auch Vermutungen, daß er noch deutlich kleiner, nämlich $\approx 10 \mathrm{~km}$, ist (Sanchez-Almeida, 1998).
} 
genannten Methoden ein Auflösungsvermögen von ca. $0.2^{\prime \prime}$, das entspricht etwa $150 \mathrm{~km}$ auf der Sonnenoberfläche. Also werden wohl zur Zeit noch keine einzelnen, sonder eher Konglomerate aus mehreren Flußröhren beobachtet. Damit ist der direkte Vergleich mit Beobachtungen noch nicht möglich.

Die Existenz von Flußröhrenkonglomeraten führt zu der Frage nach der Wechselwirkung dieser Flußröhren untereinander und mit der Umgebung. Diese Frage kann hier nicht untersucht werden, da das umgebende Medium als statisch angenommen wurde. Eine Untersuchung, die solche Wechselwirkungen mit einschlösse, wäre nicht einfach, da dann das umgebende Medium nicht mehr als räumlich eindimensional und zeitlich konstant zu behandeln wäre. 


\section{Literaturverzeichnis}

Achterberg, A.: 1988, Astron. Astrophys. 191, 167 廿

Ascher, U. M., Mattheij, R. M. M., und Russel, R. D.: 1995, Numerical Solution of Boundary Value Problems for Ordinary Differetial Equations, Society for Industrial and Applied Mathematics, Philadelphia 44

Beckers, J. M. und Schröter, E. H.: 1969, Sol. Phys. 10, 383

Bernstein, I. B., Frieman, E. A., Kruskal, M. D., und Kulsrud, R. M.: 1958, Proc. R. Soc. London, Ser. A 244, 17

Bourne, D. E. und Kendall, P. C.: 1973, Vektoranalysis, B. G. Teubner, Stuttgart 9. 10

Broyden, J. E.: 1965, Mathematics of Computation 19, 577

Caligari, P., Moreno-Insertis, F., und Schüßler, M.: 1995, Astrophys. J. 441, 8865

Cash, J. R. und Karp, A. H.: 1990, ACM Transactions on Mathematical Software 16, 201 16, 44,

Degenhardt, D.: 1989, Astron. Astrophys. 222, 297 6, 18

Degenhardt, D.: 1990, Doktorarbeit, Georg-August-Universität Göttingen 6, 7, 18, 78

Degenhardt, D.: 1991, Astron. Astrophys. 248, 637 6, 18

Degenhardt, D., Solanki, S. K., Montesinos, B., und Thomas, J. H.: 1993, Astron. Astrophys. 279, L29 18

Degenhardt, U.: 1995, Doktorarbeit, Georg-August-Universität Göttingen 4, 6, 6

Denker, C.: 1998, Sol. Phys. 180, 8180

Evershed, J.: 1909, Mon. Not. R. Astron. Soc. 69, 454 廿

Ferriz-Mas, A. und Moreno-Insertis, F.: 1987, Astron. Astrophys. 179, 268 14, 20

Frieman, E. und Rotenberg, M.: 1960, Rev. Mod. Phys. 32, 898 28, 40, 49, 51

Herbold, G., Ulmschneider, P., Spruit, H. C., und Rosner, R.: 1985, Astron. Astrophys. 145, 157 144 
Kippenhahn, R. und Möllenhoff, C.: 1975, Elementare Plasmaphysik, Bibliographisches Institut, Zürich 8

Kippenhahn, R. und Schlüter, A.: 1957, Z. Astrophys. 43, 63 廿

Kuperus, M. und Raadu, M. A.: 1974, Astron. Astrophys. 31, 189 廿

Meyer, F. und Schmidt, H. U.: 1968, Z. Ang. Math. Mech. 48, 218 5

Montesinos, B. und Thomas, J. H.: 1989, Astrophys. J. 402, 314 6, 18

Montesinos, B. und Thomas, J. H.: 1993, Astrophys. J. 337, 97718

Moreno-Insertis, F., Schüßler, M., und Ferriz-Mas, A.: 1996, Astron. Astrophys. 312, 317 29

Prautzsch, T.: 1997, Doktorarbeit, Georg-August-Universität Göttingen 5

Press, W. H., Teukolsky, S. A., Vetterling, W. T., und Flannery, B. P.: 1992, Numerical Recipes in $C$, Cambridge University Press, 2. edition [16, 41, 42, 43, 44

Roberts, B. und Webb, A. R.: 1978, Sol. Phys. 56, 5

Sanchez-Almeida, J.: 1998, Astrophys. J. 497, 96780

Schmitt, D.: 1995, Habilitationsschrift, Georg-August-Universität Göttingen 6, 28, 28, 29, 39, 40, 42, 51, 78, 78

Schmitt, D.: 1998, Geophys. Astrophys. Fluid Dyn. 89, 75 6, 28, 28, 29, 29, 30, 39, 40, 40, 42, 51, 78, 78

Schüßler, M.: 1990, Habilitationsschrift, Georg-August-Universität Göttingen 6

Spiegel, E. A. und Weiss, N. O.: 1980, Nature 287, 616 5

Spruit, H. C.: 1981a, Astron. Astrophys. 98, 155

Spruit, H. C.: 1981b, Astron. Astrophys. 102, 1296

Stix, M.: 1989, The Sun, Springer-Verlag, Berlin Heidelberg New York London Paris Tokyo

Sütterlin, P. und Wiehr, E.: 1998, Astron. Astrophys. 336, 36780

Thomas, J. H.: 1988, Astrophys. J. 333, 407

Thomas, J. H. und Montesinos, B.: 1990, Astrophys. J. 359, 550 17, 18

Thomas, J. H. und Montesinos, B.: 1991, Astrophys. J. 375, 404 6, 7, 14, 15, 18, 78 
Thomas, J. H. und Montesinos, B.: 1993, Astrophys. J. 407, 398 5, 11]

Wiehr, E.: 1999, in B. Schmieder, A. Hofmann, und J. Staude (eds.), Third advances in Solar physics euroconference: Magnetic fields and oscillations, Bd. $184 \mathrm{der}$ ASP Conference Series, S. 865 


\section{Dank}

Ich danke für die Themenstellung und Betreuung dieser Arbeit den Herren W. Deinzer und D. Schmitt. In der Endphase hat mir Herr E. Wiehr aktuelle Beobachtungsfakten in Bezug auf auf die Sonne vermittelt, auch hierfür herzlichen Dank.

Die Mitglieder der Arbeitsgruppe von Herrn Deinzer, namentlich Ulrich Degenhardt, Konstantinos Papastathis, Tilmann Prautzsch, Eric Schreiber, Martin Schrinner und Alexander Vögler haben sich besonderen Dank verdient. Die Diskussionen über Physik und andere Dinge des Lebens machen mir die Zeit in der Sternwarte zu einer schönen Erinnerung. Auch möchte ich denjenigen meine Dankbarkeit aussprechen, die mir mir bei Fragen zu Rechtschreibung und Zeichensetzung geholfen haben.

Eric Schreiber und Pit Sütterlin, mit denen ich das Workstation-Cluster des Kerninstituts der Sternwarte betreut habe, danke ich für ihre Zusammenarbeit.

Dank haben sich auch meine Eltern verdient, die mir das Studium der Physik erst ermöglicht haben.

Diese Arbeit wurde durch das Graduiertenkolleg „Strömungsinstabilität und Turbulenz“ an der Universität Göttingen gefördert. 